\title{
(2) conctere
}

\section{OPEN ACCESS}

\section{Disrupted reward processing in Parkinson's disease and its relationship with dopamine state and neuropsychiatric syndromes: a systematic review and meta-analysis}

\author{
Harry Costello 다, ${ }^{1}$ Alex J Berry, ${ }^{2}$ Suzanne Reeves (ㄷ), ${ }^{2}$ Rimona SWeil (1), ${ }^{3}$ \\ Eileen M Joyce (ㄷ), ${ }^{3}$ Robert Howard (1) , ${ }^{2}$ Jonathan P Roiser (i) ${ }^{1}$
}

\begin{abstract}
- Additional supplemental material is published online only. To view, please visit the journal online (http://dx doi.org/10.1136/jnnp-2021 327762)
\end{abstract}

${ }^{1}$ Institute of Cognitive Neuroscience, University College London, London, UK ${ }^{2}$ Division of Psychiatry, University College London, London, UK

${ }^{3}$ Institute of Neurology, University College London, London, UK

Correspondence to Dr Harry Costello, Institute of Cognitive Neuroscience, University College London, London, UK; harry.costello@ ucl.ac.uk

Received 9 August 2021 Accepted 20 November 2021

Check for updates

(C) Author(s) (or their employer(s)) 2021. Re-use permitted under CC BY. Published by BMJ.

To cite: Costello H, Berry AJ, Reeves S, et al. J Neurol Neurosurg Psychiatry Epub ahead of print: [please include Day Month Year]. doi:10.1136/jnnp-2021327762

\section{ABSTRACT}

Background Neuropsychiatric symptoms are common in Parkinson's disease (PD) and predict poorer outcomes. Reward processing dysfunction is a candidate mechanism for the development of psychiatric symptoms including depression and impulse control disorders (ICDs). We aimed to determine whether reward processing is impaired in PD and its relationship with neuropsychiatric syndromes and dopamine replacement therapy.

Methods The Ovid MEDLINE/PubMed, Embase and Psyclnfo databases were searched for articles published up to 5 November 2020. Studies reporting reward processing task performance by patients with PD and healthy controls were included. Summary statistics comparing reward processing between groups were converted to standardised mean difference (SMD) scores and meta-analysed using a random effects model.

Results We identified 55 studies containing 2578 participants (1638 PD and 940 healthy controls). Studies assessing three subcomponent categories of reward processing tasks were included: option valuation $(n=12)$, reinforcement learning $(n=37)$ and reward response vigour $(n=6)$. Across all studies, patients with PD on medication exhibited a small-to-medium impairment versus healthy controls $(S M D=0.34 ; 95 \% \mathrm{Cl}$ 0.14 to 0.53 ), with greater impairments observed off dopaminergic medication in within-subjects designs ( $\mathrm{SMD}=0.43,95 \% \mathrm{Cl} 0.29$ to 0.57). Within-subjects subcomponent analysis revealed impaired processing off medication on option valuation $(\mathrm{SMD}=0.57$, $95 \% \mathrm{Cl} 0.39$ to 0.75 ) and reward response vigour ( $\mathrm{SMD}=0.36,95 \% \mathrm{Cl} 0.13$ to 0.59 ) tasks. However, the opposite applied for reinforcement learning, which relative to healthy controls was impaired on-medication (SMD $=0.45,95 \% \mathrm{Cl} 0.25$ to 0.65 ) but not offmedication (SMD $=0.28,95 \% \mathrm{Cl}-0.03$ to 0.59 ). ICD was the only neuropsychiatric syndrome with sufficient studies ( $n=13)$ for meta-analysis, but no significant impairment was identified compared tonon-ICD patients (SMD $=-0.02,95 \% \mathrm{Cl}-0.43$ to 0.39 ).

Conclusion Reward processing disruption in PD differs according to subcomponent and dopamine medication state, and warrants further study as a potential treatment target and mechanism underlying associated neuropsychiatric syndromes.

\section{INTRODUCTION}

Parkinson's disease (PD) is the fastest growing neurological disorder globally, ${ }^{1}$ with estimated annual societal costs comparable to those of dementia. ${ }^{2}$ Traditionally conceptualised as a movement disorder, non-motor symptoms, including disruptions to mood, cognition and motivation, are common and have a greater negative impact on health-related quality of life than motor symptoms. ${ }^{3}$ Neuropsychiatric syndromes are common in PD (see table 1). One-third of patients experience depression, ${ }^{4}$ up to one-half experience apathy ${ }^{5}$ and impulse control disorders (ICDs) associated with dopaminergic medication occur in up to onequarter. ${ }^{6}$ Currently, there is a lack of understanding of the mechanisms underlying psychiatric symptoms in PD and this represents a barrier to the development of more effective treatments.

Reward processing describes how reinforcementrelated perceptions guide goal-directed behaviours. ${ }^{8}$ Impaired reward processing is a prominent transdiagnostic feature of several mental health disorders such as depression ${ }^{8}$ and represents a useful framework for understanding symptoms associated with motivation. The National Institute of Mental Health's Research Domain Criteria identifies reward processing as one of six major domains underpinning human functioning and psychopathology. ${ }^{9}$ Dopamine has a well-established role in both reward and motivational pathways. ${ }^{10}$ Evidence from dopamine depletion studies has not supported the hypothesis that dopamine mediates hedonic responses ('liking'), but has revealed a crucial role in motivated behaviours toward desired goals ('wanting'). ${ }^{11}$

PD is caused by dopaminergic cell death and consequently is a model of striatal and dopamine dysfunction. ${ }^{12}$ The striatum is reciprocally connected with prefrontal areas as well as other parts of the basal ganglia and midbrain, forming frontostriatal circuits involved in the initiation and control of motor, cognitive and emotional function. These pathways also constitute part of the brain's reward circuit, responsible for modulating rewardrelated behaviour and learning. ${ }^{13}$ Psychiatric syndromes in PD (see table 1) are thought to reflect dysfunction of non-motor frontostriatal circuitry; for example, ICDs are believed to develop through 
Table 1 . Current understanding of the role of reward processing in neuropsychiatric symptoms \& syndromes in PD.

\begin{tabular}{|c|c|c|}
\hline Common PD neuropsychiatric symptoms and syndromes & Prevalence in PD & Relationship with reward processing \\
\hline $\begin{array}{l}\text { Apathy-loss or reduction of motivation compared with an } \\
\text { individual's previous state. }\end{array}$ & $40 \%^{\mathrm{s1}}$ & \multirow{2}{*}{$\begin{array}{l}\text { Apathy and anhedonia are disorders of motivation. Effort-based decision making for } \\
\text { reward, the process of how a potential benefit/reward for performing an activity is } \\
\text { evaluated with respect to the cost in effort required to attain it, is believed to be a key } \\
\text { reward processing mechanism underlying both symptoms. } .^{33}\end{array}$} \\
\hline $\begin{array}{l}\text { Anhedonia-consistently diminished interest or pleasure in } \\
\text { almost all daily activities. }\end{array}$ & $46 \%{ }^{\mathrm{s2}}$ & \\
\hline $\begin{array}{l}\text { Depression—clinical syndrome with core symptoms of } \\
\text { persistent low mood and anhedonia. }\end{array}$ & $20 \%-30 \%{ }^{54,55}$ & $\begin{array}{l}\text { Disrupted reward processing is understood to be a key cognitive mechanism underlying } \\
\text { depressive symptoms. Patients with depression have been shown to have impaired option } \\
\text { valuation, reinforcement learning and reward bias versus healthy controls. } .^{56}\end{array}$ \\
\hline $\begin{array}{l}\text { Anxiety — often co-morbid with depression, symptoms include } \\
\text { persistent tension, worry and feelings of apprehension. }\end{array}$ & $25 \%{ }^{57}$ & $\begin{array}{l}\text { Individuals with anxiety are less sensitive to rewards depending on certainty, preferring } \\
\text { less profitable but more predictable options over riskier more rewarding outcomes. } .^{88}\end{array}$ \\
\hline $\begin{array}{l}\text { Impulse control disorder (ICD)—development of harmful risk- } \\
\text { taking and impulsive behaviours. Can include pathological } \\
\text { gambling, hypersexuality and sudden episodes of aggression } \\
\text { (intermittent explosive disorder). }\end{array}$ & $25 \%-30 \%{ }^{\mathrm{s3}}$ & $\begin{array}{l}\text { ICD has been proposed to be secondary to dopamine agonists and Parkinson's pathology } \\
\text { sensitising patients to reward. }{ }^{59} \text { Increased reward sensitivity is suggested to then lead to } \\
\text { immediate reward seeking behaviours and impulsivity. }\end{array}$ \\
\hline $\begin{array}{l}\text { Dopamine dysregulation syndrome-complication of PD } \\
\text { treatment characterised by addictive behaviour and excessive } \\
\text { use of dopaminergic medication. }\end{array}$ & $3 \%-4 \%$ & $\begin{array}{l}\text { The reward deficiency theory of addiction posits that patients have a deficit in recruiting/ } \\
\text { hypoactivation of striatal reward pathways, leading to compensatory addictive behaviours } \\
\text { such as drug seeking. Striatal hypoactivation during reward anticipation has been found in } \\
\text { individuals with addiction. } \text {.11 }^{10}\end{array}$ \\
\hline $\begin{array}{l}\text { Psychosis—used to describe range of hallucinations and } \\
\text { delusions. }\end{array}$ & $\begin{array}{l}\text { Visual:: }: 12 \\
22 \%-38 \% \\
\text { Auditory: }{ }^{512} 20 \% \\
\text { Delusions: }{ }^{.12} 5 \%\end{array}$ & $\begin{array}{l}\text { Abnormal reward processing driven by elevated ventral striatal dopamine levels is } \\
\text { hypothesised to underlie psychotic symptoms. Hypoactivation of the ventral striatum } \\
\text { during reward anticipation has been reported in psychosis. }{ }^{513}\end{array}$ \\
\hline
\end{tabular}

See online supplement for references.

PD, Parkinson's disease.

aberrant reward processing, due to an interaction between the disrupted reward processing circuitry underlying PD and dopamine agonist treatment. ${ }^{14}$

Over the past two decades, studies of reward processing in PD have typically used behavioural tasks assessing three subcomponent processes ${ }^{8}$ : (1) option valuation, the process by which individuals evaluate reward-related options when given explicit information about those options (eg, reward, cost and probability); (2) reward response vigour, which reflects the speed or strength with which an individual executes an action to obtain a reward; (3) reinforcement learning, which describes the process by which an individual uses feedback to change their future behaviour. To date, there has been one meta-analysis of Iowa gambling task performance in PD, which reported significantly impaired reward learning. ${ }^{15}$ However, the degree and pattern of impairments on other reward processing tasks in PD and any relationship with dopaminergic state and psychiatric symptoms remain unclear.

Here we report the first systematic review and meta-analysis of reward processing behaviour in PD and its relationship with dopamine replacement therapy and associated neuropsychiatric syndromes. Our aims were: (1) to clarify the nature and extent of differences across reward processing subcomponents between PD and healthy groups; (2) to test the role of dopamine state (on or off medication) in reward processing in PD; (3) to investigate any differences in reward processing in patients with PD with and without neuropsychiatric syndromes.

\section{METHOD}

\section{Systematic review}

The Ovid MEDLINE/PubMed, Embase, and PsycInfo databases were searched for articles published between 1 January 1946 and 5 November 2020 inclusive, with titles or abstracts containing the terms: Parkins* and (reward* or motivat" or incentiv* or effort" or deci*) and (psychiatric or neuropsychiatric or depress" or psychosis or delus" or impuls" or mood or anxiety or apathy or anhedonia or hallucin*). Inclusion criteria were as follows: (1) case-control design; (2) included a group with PD without dementia or deep brain stimulation (DBS) (studies including participants with dementia or DBS withing the PD group were excluded); (3) participants were at least 18 years old; (4) participants performed a reward-processing task; (5) task rewards were explicit, that is, money, points, water or food (we did not include studies that used outcomes that could be considered purely informational or social feedback, eg, happy/sad faces or variants of correct/incorrect, to ensure specificity); (6) studies reported data on a behavioural measure of reward processing that could be converted to a case-control standardised mean difference (SMD) score. If this was not reported, data were requested from the authors. Articles were independently assessed by HC and AJB, using a rating tool based on the Newcastle-Ottawa scale ${ }^{16}$ for assessing the quality of non-randomised studies (online supplement). Conflicts in quality assessment rating were resolved through in-person discussion.

\section{Meta-analysis}

Behavioural measures from each study were categorised as measuring option valuation, reward response vigour or reinforcement learning, and converted to an SMD score and an associated SE (see online supplemental material for equations). ${ }^{17}$

Within the option valuation and reward response vigour subcategories, a positive SMD represents a greater or faster response to reward by the control than the PD group, respectively. A positive SMD within the reinforcement learning subcategory represents faster use of feedback to maximise reward by the control group than the PD group.

Meta-analysis was conducted if four or more studies were present within a reward processing subcategory for patients with PD compared with healthy controls, PD with and without a psychiatric symptom, or PD on-medication compared with offmedication (within-subjects designs only).

Meta-analysis was performed using the $\mathrm{R}$ statistical programming language and the packages metafor and metaviz, using random effects models. Heterogeneity was analysed using the approximate proportion of total variability $\left(\mathrm{I}^{2}\right)$. 


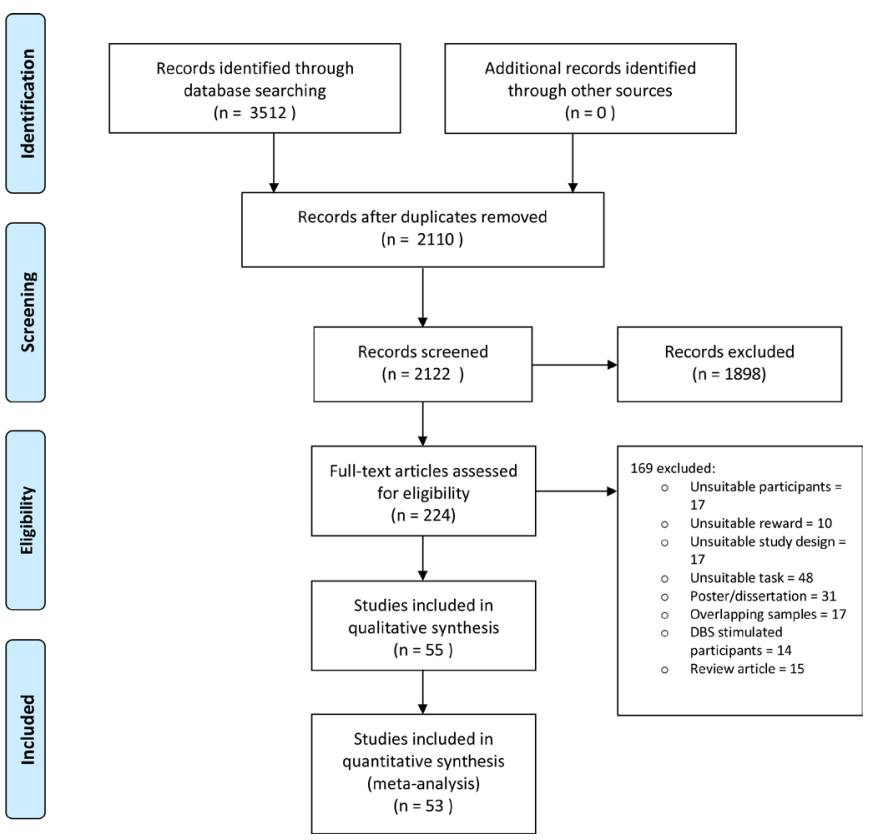

Figure 1 PRISMA flow diagram of study selection and inclusion. DBS, deep brain stimulation; PRISMA, Preferred Reporting Items for Systematic Reviews and Meta-Analyses.

Funnel plot asymmetry was assessed using visual inspection of a contour-enhanced funnel plot and the Egger test.

\section{RESULTS}

We initially identified 2122 studies, excluded 1898 of these by title/abstract and retrieved the remaining 224 full papers (figure 1). Data from 55 studies containing 2578 participants (1638 PD, 940 healthy controls) were analysed (see Preferred Reporting Items for Systematic Reviews and Meta-Analyses diagram in figure 1); two studies could not be used in the quantitative analysis due to a lack of reported summary statistics. The median number of patients per study was 24 (IQR 16), median participant age was 63.3 years (IQR 7.5) and median duration of PD was 7.0 years (IQR 4.5).

Meta-analysis across all reward processing subcomponent categories (see online supplemental table 4) identified a smallto-medium reward processing impairment in patients with PD both on-medication (SMD $=0.34 ; 95 \%$ CI 0.14 to 0.53 ) and offmedication (SMD $=0.40 ; 95 \%$ CI 0.19 to 0.62 ), compared with healthy controls (figure 2A, B). Within-subjects comparison of reward processing between on-medication and off-medication states was possible in 14 studies (see online supplemental table 6), revealing relatively impaired reward processing off-medication, with a medium effect size $(\mathrm{SMD}=0.43,95 \% \mathrm{CI} 0.29$ to 0.57 ; figure $3 \mathrm{~A})$.

ICD was the most studied and only neuropsychiatric syndrome with sufficient studies $(n=13)$ for meta-analysis (see online supplemental table 5). No significant impairment (see figure 3B) was identified in reward processing in patients with PD with ICD compared to patients with non-ICD $(\mathrm{SMD}=-0.02,95 \% \mathrm{CI}$ -0.43 to 0.39 ).

Overall interstudy heterogeneity was substantial $\left(\mathrm{I}^{2}=57.48 \%\right)$, and the median power of included studies and R-index was low (online supplemental figure 1), median power $=36 \%$; index $=28 \%$ ). Analysis of funnel plot asymmetry using Egger's regression line did not meet statistical significance $(p=0.32)$ and was likely a consequence of high heterogeneity and small sample size of included studies (see online supplemental figure 1).

Quality assessment and risk of bias analysis using a modified Newcastle-Ottawa scale (see online supplemental table 7) found the majority of included studies used a validated assessment tool for diagnosis of PD (65.5\%), and accounted for PD severity $(94.5 \%)$ and medication status (90.9\%). However, almost half of included studies gave no description of how healthy controls were selected $(42.2 \%)$ or clearly defined controls as having no past psychopathology (42.2\%).

\section{Option valuation}

We identified 12 studies containing 347 patients with PD and 278 healthy participants that used option valuation tasks (online supplemental table 1). The mean age of participants was 62.9 $( \pm 4.6)$ years, and mean duration of illness was $7.5( \pm 2.8)$ years. Effort-based decision-making tasks (three studies) and the game of dice task (three studies) were most commonly used. Four studies reported psychiatric medication use in participants, three of which included participants taking antidepressant medications.

Meta-analysis of studies comparing option valuation in patients with PD compared with healthy controls showed lower reward weighting in $\mathrm{PD}$, which was moderated by dopamine
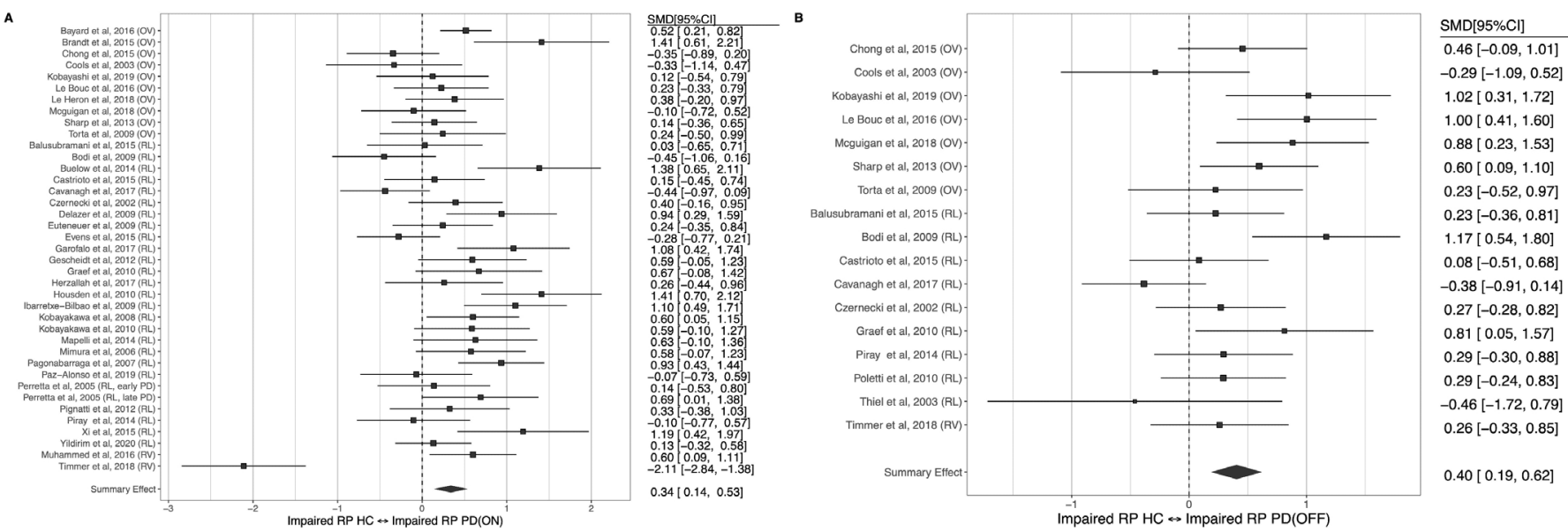

Figure 2 Forest plot of reward processing (RP) in (A) PD ON versus healthy controls (HC), (B) PD OFF versus HC. PD, Parkinson's disease; SMD, standardised mean difference. 


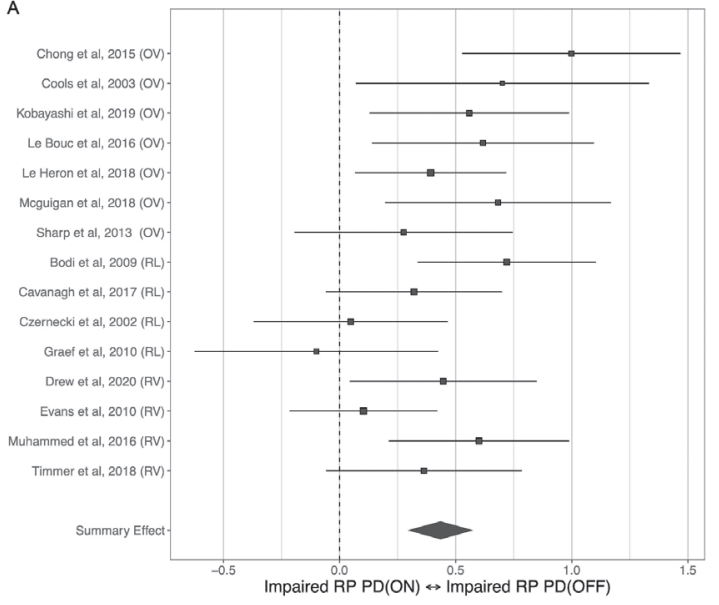

$\mathrm{SMD}[95 \% \mathrm{Cl}]$

$1.00[0.53,1.47]$

$0.70[0.07,1.33]$

$0.56[0.13,0.99]$

$0.62[0.14,1.10]$

$0.39[0.07,0.72]$

$0.68[0.20,1.17]$

$0.28[-0.19,0.75]$

$0.72[0.34,1.10]$

$0.32[-0.06,0.70]$

$0.05[-0.37,0.47]$

$-0.10[-0.63,0.43]$

$0.45[0.04,0.85]$

$0.10[-0.22,0.42]$

$0.60[0.21,0.99]$

$0.36[-0.06,0.78]$

$0.43[0.29,0.57]$

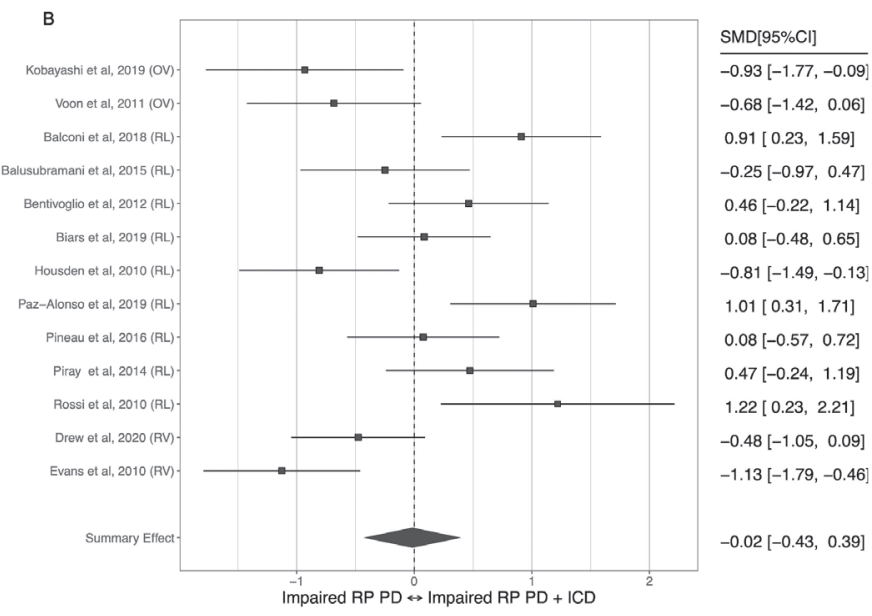

Figure 3 Forest plot of reward processing (RP) in (A) PD ON versus OFF dopamine state, (B) PD with and without impulse control disorder (ICD). PD, Parkinson's disease; SMD, standardised mean difference.

medication (figure 4A, B). Patients on-medication did not differ significantly from healthy controls $(\mathrm{SMD}=0.22,95 \% \mathrm{CI}$ -0.04 to 0.49 ), but off-medication there was a medium-to-large impairment $(\mathrm{SMD}=0.60,95 \% \mathrm{CI} 0.30$ to 0.89$)$. Within-subjects comparison confirmed lower reward weighting off-medication, with a medium-to-large effect $(\mathrm{SMD}=0.57,95 \% \mathrm{CI} 0.39$ to 0.75 ; figure $4 \mathrm{C})$.

Four studies compared option valuation in patients with PD with and without neuropsychiatric syndromes. Three of these studies ${ }^{18-20}$ compared option valuation in patients with PD with and without ICD, with mixed findings. One study ${ }^{19}$ using an economic choice task reported lower reward weighting in ICD, while the other two studies ${ }^{18} 20$ using gambling tasks found no difference ${ }^{18}$ and increased reward weighting, ${ }^{20}$ respectively.

One study ${ }^{21}$ investigating the effect of apathy on option valuation reported lower acceptance of offers of reward obtained through physical exertion. This pattern of impairment in apathy was found to be dissociable from the effects of dopamine. Apathy was characterised by rejection of predominantly low reward offers, while dopamine state increased choices of high effort, high reward offers.

In summary, option valuation impairment in PD is dopamine dependent, with lower reward weighting off dopaminergic medication. Too few studies have investigated option valuation in patients with PD with neuropsychiatric syndromes to draw meaningful conclusions.

\section{Reinforcement learning}

We identified 37 studies containing 1059 patients with PD and 593 healthy controls that used reinforcement learning tasks (online supplemental table 2). The majority of studies (20/37)

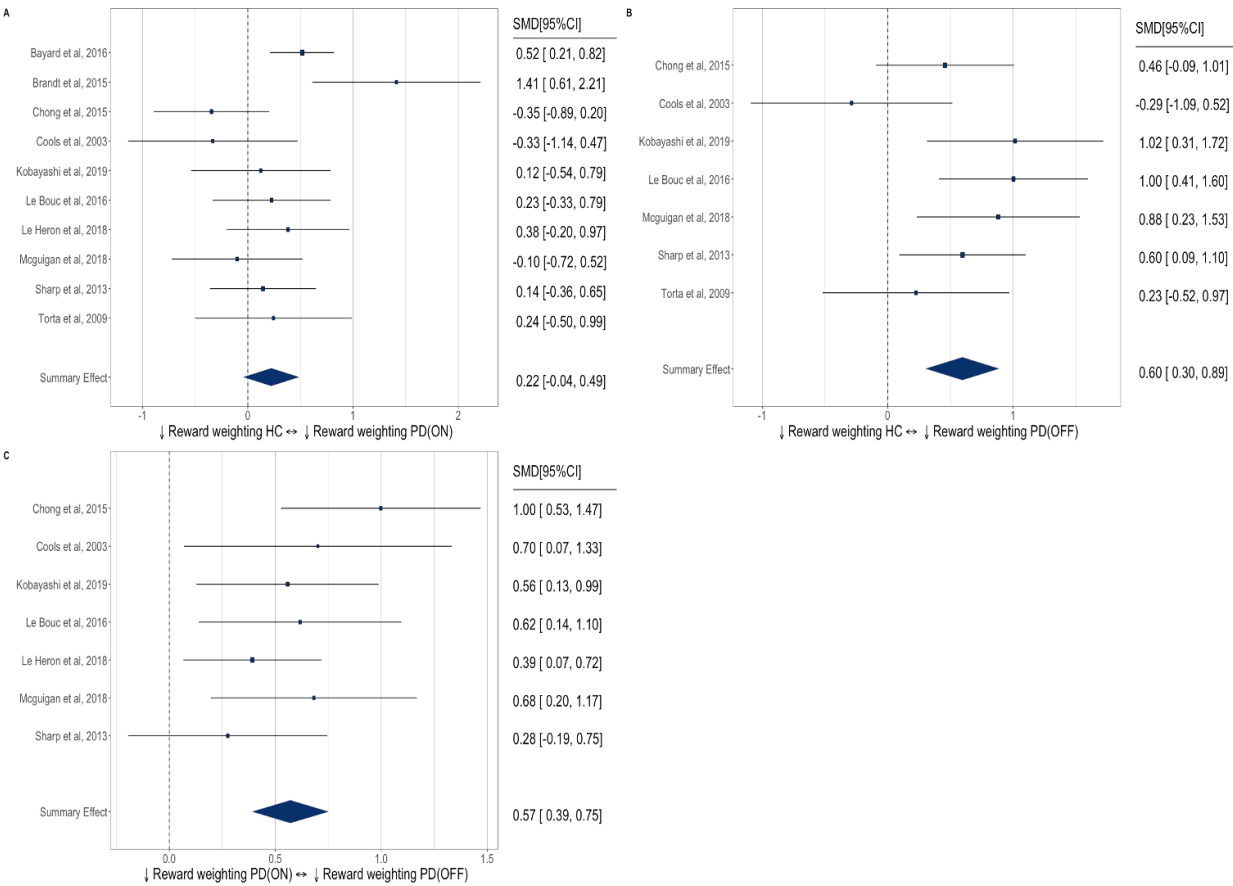

Figure 4 Forest plot of option valuation in: (A) PD ON versus healthy controls, (B) PD OFF versus healthy controls, (C) PD ON versus OFF dopamine state. $\mathrm{HC}$, healthy controls; PD, Parkinson's disease; SMD, standardised mean difference. 


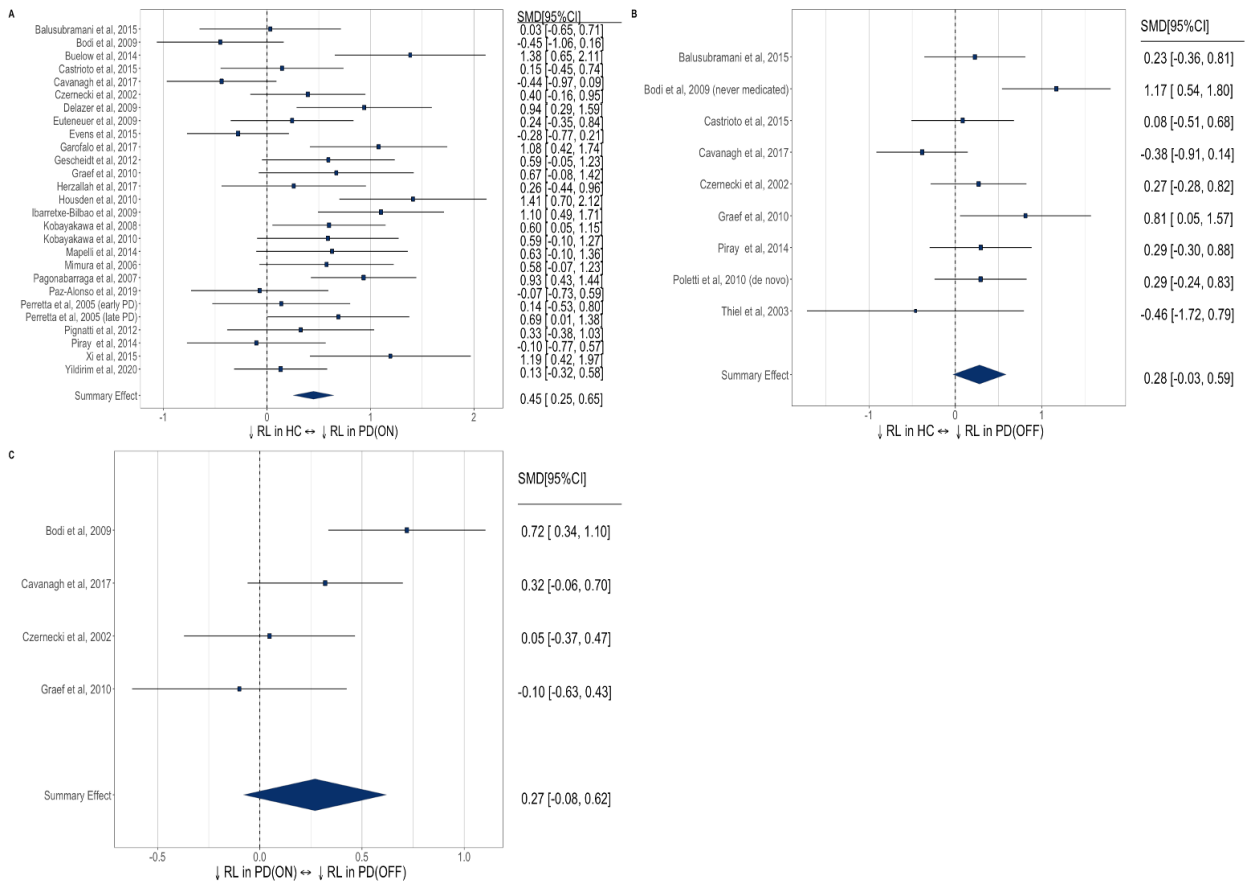

Figure 5 Forest plot of reinforcement learning (RL) in: (A) PD ON versus healthy controls, (B) PD OFF versus healthy controls, (C) PD ON versus OFF dopamine state. $\mathrm{HC}$, healthy controls; PD, Parkinson's disease; SMD, standardised mean difference.

used the Iowa gambling task. Ten studies reported psychiatric medication use, of which three included participants taking antidepressant medication.

Reinforcement learning was slowed in patients with PD on-medication versus healthy controls (figure $5 \mathrm{~A}, \mathrm{~B}$ ) with a medium effect size ( $\mathrm{SMD}=0.45,95 \% \mathrm{CI} 0.25$ to 0.65$)$. Interestingly, there was no significant group difference off-medication ( $\mathrm{SMD}=0.28,95 \% \mathrm{CI}-0.03$ to 0.59 ). Comparison of reinforcement learning comparing on-medication and off-medication within-subjects (figure $5 \mathrm{Cc}$ ) was possible in four studies, which did not detect a significant effect $(\mathrm{SMD}=0.27,95 \% \mathrm{CI}-0.08$ to 0.62 ); however, we note that this analysis is likely underpowered due to the small number of included studies.

Sixteen studies investigated reinforcement learning in patients with PD with and without neuropsychiatric symptoms (online supplemental table 2), with the majority (11/16) examining ICD. Meta-analysis of nine studies (online supplemental figure 2) found no significant difference between patients with PD with ICD and non-ICD PD patients (SMD $=0.32,95 \% \mathrm{CI}-0.09$ to 0.73 ).

Two studies ${ }^{22} 23$ examined reinforcement learning in patients with PD with major depressive disorder. Both ${ }^{22}{ }^{23}$ reported impaired reinforcement learning in depressed patients with PD compared with non-depressed patients with PD. One ${ }^{23}$ also compared reinforcement learning in depressed patients with PD with depressed participants without PD. A similar pattern of impairment in learning from positive feedback was identified in the two groups, suggesting that reinforcement learning impairment may not be specific to depression in PD. ${ }^{9}$

Two studies ${ }^{2425}$ examined the role of apathy in reward learning. Both used the Iowa gambling task but reported conflicting findings: one found significant impairment ${ }^{25}$ but the other reported better reinforcement learning in patients with PD with apathy, ${ }^{24}$ compared with those without.

In summary, and in stark contrast to studies of option valuation, reinforcement learning is particularly impaired in PD in the on-medication state. There was no significant impairment in reinforcement learning in patients with PD with ICD compared with those without ICD. Too few studies have investigated reinforcement learning in patients with PD with other neuropsychiatric syndromes to draw meaningful conclusions.

\section{Reward response vigour}

We identified seven studies containing 232 patients with PD and 69 healthy controls that investigated reward response vigour in PD (online supplemental table 3). Insufficient studies were identified to allow meta-analysis of reward response vigour in PD compared with healthy controls. Of the three studies ${ }^{26-28}$ that reported reward response vigour in PD and healthy controls, results were mixed, with studies reporting lower, ${ }^{26}$ greater $^{27}$ and no difference ${ }^{28}$ in patients with PD compared with healthy volunteers.

Meta-analysis of the effect of dopamine state on reward response vigour in four studies (figure 6) identified a smallto-medium increase in reward response vigour on-medication ( $\mathrm{SMD}=0.36,95 \%$ CI 0.13 to 0.59 ).

Six studies investigated reward response vigour in patients with PD with and without neuropsychiatric syndromes (online supplemental table 3). Two studies ${ }^{27}{ }^{29}$ examined apathy, one using a rewarded saccadic eye movement task, ${ }^{27}$ the other a rewarded spatial search task ${ }^{29}$; both reported no significant group differences. Similarly, no significant difference in reward response vigour was found in two studies comparing patients with ICD and patients with non-ICD, ${ }^{3031}$ and two investigating depression in PD. ${ }^{28}$

In summary, relatively few studies have investigated reward response vigour in $\mathrm{PD}$, and findings are mixed. Reward response vigour in PD was reduced in the off-medication compared with the on-medication state. Too few studies have investigated reward response vigour in patients with PD with neuropsychiatric syndromes to draw meaningful conclusions. 


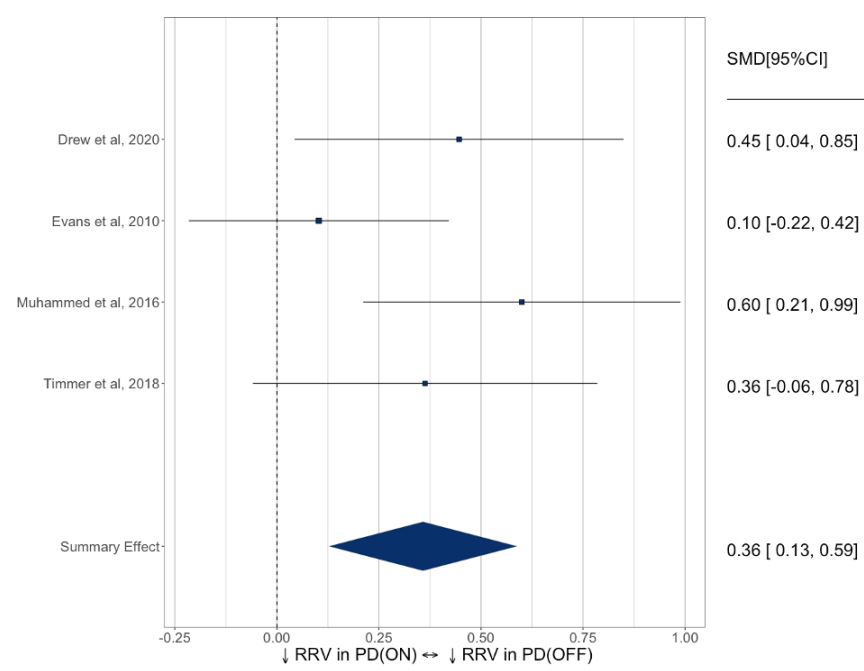

Figure 6 Forest plot of reward response vigour (RRV) in PD ON versus OFF dopamine state. PD, Parkinson's disease; SMD, standardised mean difference.

\section{DISCUSSION}

This is the first systematic review and meta-analysis of reward processing in $\mathrm{PD}$, associated neuropsychiatric syndromes and the influence of dopaminergic medication. Across all 55 studies, including different subcomponents of reward processing, we found patients with PD to have small-to-medium reward processing impairments relative to healthy participant groups. The degree of impairment in reward processing is similar to that reported in major depressive disorder, a condition where dysfunctional reward processing is a leading aetiological candidate mechanism for 'interest-activity' symptoms, such as anhedonia. ${ }^{8}$ We also identified potentially important differences between reward processing subcomponent categories and the effect of dopamine state.

The option valuation subcategory exhibited the largest impairment in PD which was dopamine dependent, with markedly reduced reward weighting in patients with $\mathrm{PD}$ off dopaminergic medication. This finding is supported by animal ${ }^{11}$ and human experimental studies ${ }^{32}$ which show impaired valuation following dopamine depletion. Dopamine antagonists such as antipsychotic drugs also reduce preference for high-effort/high-reward options, ${ }^{11}$ suggesting that dopamine transmission is crucial in cost-benefit decision making. Dopaminergic pathways in the brain reward circuit including the anterior cingulate cortex and basal ganglia are believed to be central in choosing and executing effortful action. ${ }^{5}$ Option valuation is a component of effortbased decision making and represents a framework for understanding apathy and anhedonia, both common motivational disorders in PD and depression. ${ }^{5}$ However, no study to date has investigated option valuation in depression in PD, and the only study ${ }^{21}$ to examine apathy found dissociable effects of dopamine and apathy on decision making, indicating impairment may not only be secondary to dopamine depletion.

In direct contrast to the pattern identified in the option valuation subcategory, reinforcement learning was moderately impaired in PD when patients were on dopamine medication, with no significant difference detected when off medication. This is surprising given decades of evidence that dopaminergic pathways from the midbrain are crucial for reward learning. ${ }^{33}$ However, recent studies applying cell-type specific monitoring and manipulation of distinct neuronal populations in the striatum have suggested that heterogenous signals in dopaminergic neurons support specific types of learning. ${ }^{34}$ For example, differentially regulated mechanisms of dopamine release in the basal ganglia underlie distinct functions. ${ }^{35}$ Reward learning is believed to be facilitated by dopamine cell spiking encoding reward prediction errors, whereas gradual increase in dopamine release mirrors reward expectation. ${ }^{35}$ Reinforcement learning is therefore believed to be dependent on phasic rather than tonic dopamine signalling. Wave-like spatiotemporal dopamine dynamics in the dorsal striatum have also been implicated in encoding reward prediction errors to facilitate learning. ${ }^{36}$ It remains unclear what effect exogenous dopamine in PD has on the dynamics of striatal dopamine signalling. Studies of associative learning in healthy subjects have found that dopamine agonists can impair learning by inhibiting phasic dopamine signalling. ${ }^{37}$ Therefore, one possible interpretation is that dopamine medication may remediate control of reward expectation and motivation within the striatum, but impair the broadcast burst signals required to promote learning. ${ }^{35}$ However, this requires testing in future studies.

Distinct types of reinforcement learning model used during task performance may also play a crucial role. ${ }^{38}$ 'Model-free' learning describes learning through direct experience rather than through constructing an internal model of the environment in order to develop a complex map of cues and actions which lead to reward. ${ }^{38}$ Most studies included in our review used model free reinforcement learning tasks. Evidence suggests that these two types of reinforcement learning processes are mechanistically distinct, and differentially dependent on dopamine reward prediction errors. $^{38}$

The reward response vigour subcategory showed a significant small-to-moderate impairment in the off-medication compared with the on-medication state in patients with PD. However, relatively few studies were identified and reaction times may be vulnerable to attentional confounds. Though several studies reported reaction times during tasks, reward-related speeding (ie, the difference between rewarded and non-reward conditions) was infrequently measured, without which slower reaction times would likely only reflect bradykinesia associated with PD.

Despite PD being a model for dopamine dysfunction, current treatments of common neuropsychiatric syndromes in PD such as depression do not differ from depression in patients with other long-term conditions ${ }^{39}$ and have limited efficacy. ${ }^{40}$ Symptoms of anxiety and depression in patients with PD with motor fluctuations can be more common and severe in the off-dopamine state, ${ }^{41}$ suggesting depression in PD may be related to dopaminergic deficit and have a specific aetiology. Our findings suggest PD is characterised by a specific pattern of impairment in reward processing which is dopamine dependent and potentially could be a causal mechanism underlying neuropsychiatric symptoms such as depression. Although ICDwas not significantly associated with reward processing impairment statistical power was limited, and few studies have investigated reward processing in other PD-associated neuropsychiatric syndromes. Further understanding of how impairment in reward processing is associated with specific neuropsychiatric manifestations of PD is needed to understand the underlying mechanisms of these disabling syndromes and develop more targeted and effective treatments.

\section{LIMITATIONS}

We categorised reward processing into three subcomponent categories, however there are several ways to measure function in each category which grouped diverse processes. For example, 
the option valuation subcategory included studies measuring risk taking and decisions to exert effort, resulting in meta-analysis of heterogeneous measures. A minority of studies reported psychiatric medication use in participants. Evidence suggests antidepressant medication may partly exert its effect via modulating reward processing ${ }^{42}$ which could have confounded results. Though we measured and compared the effect of dopamine medication state on task performance, the medication regime and proportion of patients on dopamine agonist treatment as opposed to levodopa was reported in less than half of included studies (22/55). Different PD medications are disproportionately associated with dopamine-related psychiatric conditions such as ICD ${ }^{6}$ and distinct regimes could potentially impact reward processing variably. The majority of studies investigating reward processing in PD-associated neuropsychiatric syndromes used patients with PD without the syndrome as a control group. Only one study ${ }^{23}$ investigating depression in PD used a control group of patients with depression without PD. In order to establish whether patterns of reward processing impairments are specific to PD-associated neuropsychiatric syndromes and not a common feature of psychiatric symptoms more generally, further studies of this type are needed. Finally, our systematic review and metaanalysis examined the findings of case-control studies which are unable to inform us of the causal relationship between reward processing impairment, PD and its associated neuropsychiatric syndromes. Longitudinal studies are needed to answer these questions and understand how reward processing changes develop as PD advances. Our analyses of the impact of dopamine medication were derived from studies conducted using within-subjects experimental comparisons, and therefore we can be more confident of a causal role. However, the effects of being off-medication in a patient who usually takes dopamine-boosting drugs, including heightened anxiety and physical discomfort, could plausibly affect task performance. A minority of studies $(22 / 55)$ measured motor symptom severity in both on and off states, and only four studies measured differences in anxiety symptoms in both states.

\section{CONCLUSIONS}

PD is associated with a small-to-medium level of reward processing impairment overall, with variable degrees of impairment across subcomponent reward processing categories. Reward processing is dependent on dopamine state with greater impairment in option valuation and reward response vigour when patients are off dopaminergic medication, but surprisingly faster reinforcement learning. Other than reinforcement learning in ICD, few studies have investigated the relationship between reward processing and PD associated neuropsychiatric syndromes. Further research, including longitudinal studies are needed to conclude whether specific patterns of impairment in reward processing have a causal relationship with neuropsychiatric syndromes in PD.

Correction notice This article has been corrected since it was published online first. The caption of figure 3 has been updated.

Twitter Harry Costello @harrry_costello, Suzanne Reeves@Suzanne04823062, Rimona SWeil @rimonaweil, Robert Howard @ProfRobHoward and Jonathan P Roiser @jonroiser

Contributors $\mathrm{HC}$, JPR and RH contributed to study concept and design. $\mathrm{HC}$ and AJB contributed to quality assessment of studies. $\mathrm{HC}, \mathrm{AJB}$ and JPR contributed to acquisition of data. HC and JPR contributed to analysis and interpretation of data. $\mathrm{HC}$ contributed to drafting of manuscript. All authors critically revised successive drafts of the paper and approved the final version. HC accepts responsibility as guarantor for the overall content of the study.
Funding HC is supported by a Wellcome Trust Clinical Training Fellowship (175479). Competing interests None declared.

Patient consent for publication Not applicable.

Ethics approval This study is secondary research that synthesised the results of original papers; as such, it is exempt from ethical approval.

Provenance and peer review Not commissioned; externally peer reviewed.

Data availability statement All data relevant to the study are included in the article or uploaded as supplementary information.

Supplemental material This content has been supplied by the author(s). It has not been vetted by BMJ Publishing Group Limited (BMJ) and may not have been peer-reviewed. Any opinions or recommendations discussed are solely those of the author(s) and are not endorsed by BMJ. BMJ disclaims all liability and responsibility arising from any reliance placed on the content. Where the content includes any translated material, BMJ does not warrant the accuracy and reliability of the translations (including but not limited to local regulations, clinical guidelines, terminology, drug names and drug dosages), and is not responsible for any error and/or omissions arising from translation and adaptation or otherwise.

Open access This is an open access article distributed in accordance with the Creative Commons Attribution 4.0 Unported (CC BY 4.0) license, which permits others to copy, redistribute, remix, transform and build upon this work for any purpose, provided the original work is properly cited, a link to the licence is given, and indication of whether changes were made. See: https://creativecommons.org/ licenses/by/4.0/.

\section{ORCID iDs}

Harry Costello http://orcid.org/0000-0003-4490-9219

Suzanne Reeves http://orcid.org/0000-0001-8053-7024

Rimona SWeil http://orcid.org/0000-0002-5092-6325

Eileen M Joyce http://orcid.org/0000-0003-0469-2844

Robert Howard http://orcid.org/0000-0002-3071-2338

Jonathan P Roiser http://orcid.org/0000-0001-8269-1228

\section{REFERENCES}

1 Dorsey ER, Sherer T, Okun MS, et al. The emerging evidence of the Parkinson pandemic. J Parkinsons Dis 2018;8:S3-8.

2 Weir S, Samnaliev M, Kuo T-C, et al. Short- and long-term cost and utilization of health care resources in Parkinson's disease in the UK. Mov Disord 2018;33:974-81.

3 Müller B, Assmus J, Herlofson K. Importance of motor vs. non-motor symptoms for health-related quality of life in early Parkinson's disease. Park Relat Disord 2013.

4 Reijnders JSAM, Ehrt U, Weber WEJ, et al. A systematic review of prevalence studies of depression in Parkinson's disease. Mov Disord 2008;23:183-9.

5 Husain M, Roiser JP. Neuroscience of apathy and anhedonia: a transdiagnostic approach. Nat Rev Neurosci 2018;19:470-84.

6 Antonini A, Barone P, Bonuccelli U, et al. ICARUS study: prevalence and clinical features of impulse control disorders in Parkinson's disease. J Neurol Neurosurg Psychiatry 2017;88:317-24.

7 Alzahrani H, Venneri A. Cognitive and neuroanatomical correlates of neuropsychiatric symptoms in Parkinson's disease: a systematic review. J Neurol Sci 2015;356:32-44.

8 Halahakoon DC, Kieslich K, O'Driscoll C. Reward-Processing behavior in depressed participants relative to healthy volunteers: a systematic review and meta-analysis. JAMA Psychiatry 2020:77:1286-95.

9 Insel T, Cuthbert B, Garvey M, et al. Research domain criteria (RDoC): toward a new classification framework for research on mental disorders. Am J Psychiatry 2010;167:748-51.

10 Zald DH, Treadway MT. Reward processing, Neuroeconomics, and psychopathology. Annu Rev Clin Psychol 2017;13:471-95

11 Kurniawan IT, Guitart-Masip M, Dolan RJ. Dopamine and effort-based decision making. Front Neurosci 2011;5:81.

12 Surmeier DJ, Graves SM, Shen W. Dopaminergic modulation of striatal networks in health and Parkinson's disease. Curr Opin Neurobiol 2014;29:109-17.

13 Glimcher PW. Understanding dopamine and reinforcement learning: the dopamine reward prediction error hypothesis. Proc Natl Acad Sci U S A 2011;108 Suppl 3:15647-54

14 Drew DS, Muhammed K, Baig F, et al. Dopamine and reward hypersensitivity in Parkinson's disease with impulse control disorder. Brain 2020;143:2502-18.

15 Evens R, Hoefler M, Biber K, et al. The lowa gambling task in Parkinson's disease: a meta-analysis on effects of disease and medication. Neuropsychologia 2016:91:163-72.

16 Sidwell K. Newcastle - Ottawa quality assessment scale case control studies. J Hell Stud 1993.

17 Gibbons RD, Hedeker DR, Davis JM. Estimation of effect size from a series of experiments involving paired comparisons. J. Educ. Stat 1993 
18 Haagensen BN, Herz DM, Meder D, et al. Linking brain activity during sequential gambling to impulse control in Parkinson's disease. Neuroimage Clin 2020;27:102330.

19 Kobayashi S, Asano K, Matsuda N, et al. Dopaminergic influences on risk preferences of Parkinson's disease patients. Cogn Affect Behav Neurosci 2019;19:88-97.

20 Voon V, Gao J, Brezing C, et al. Dopamine agonists and risk: impulse control disorders in Parkinson's disease. Brain 2011;134:1438-46.

21 Le Heron C, Plant O, Manohar S, et al. Distinct effects of apathy and dopamine on effort-based decision-making in Parkinson's disease. Brain 2018;141:1455-69.

22 Timmer MHM, Sescousse G, van der Schaaf ME, et al. Reward learning deficits in Parkinson's disease depend on depression. Psychol Med 2017:47:2302-11.

23 Herzallah MM, Khdour HY, Taha AB, et al. Depression reduces accuracy while parkinsonism slows response time for processing positive feedback in patients with Parkinson's disease with comorbid major depressive disorder tested on a probabilistic category-learning task. Front Psychiatry 2017:8:84

24 Martínez-Horta S, Pagonabarraga J, Fernández de Bobadilla R, et al. Apathy in Parkinson's disease: more than just executive dysfunction. J Int Neuropsycho/ Soc 2013;19:571-82.

25 Buelow MT, Frakey LL, Grace J, et al. The contribution of apathy and increased learning trials to risky decision-making in Parkinson's disease. Arch Clin Neuropsychol 2014:29:100-9

26 Renfroe JB, Bradley MM, Okun MS, et al. Motivational engagement in Parkinson's disease: preparation for motivated action. Int J Psychophysiol 2016;99:24-32

27 Muhammed K, Manohar S, Ben Yehuda M, et al. Reward sensitivity deficits modulated by dopamine are associated with apathy in Parkinson's disease. Brain 2016;139:2706-21.

28 Timmer MHM, Aarts E, Esselink RAJ, et al. Enhanced motivation of cognitive control in Parkinson's disease. Eur J Neurosci 2018:48:2374-84.

29 Lawrence AD, Goerendt IK, Brooks DJ. Apathy blunts neural response to money in Parkinson's disease. Soc Neurosci 2011;6:653-62.

30 Drew DS, Muhammed K, Baig F, et al. Dopamine and reward hypersensitivity in Parkinson's disease with impulse control disorder. Brain 2020;143:2502-18.
31 Evans $A H$, Lawrence AD, Cresswell SA, et al. Compulsive use of dopaminergic drug therapy in Parkinson's disease: reward and anti-reward. Mov Disord 2010;25:867-76.

32 Roiser JP, McLean A, Ogilvie AD, et al. The subjective and cognitive effects of acute phenylalanine and tyrosine depletion in patients recovered from depression. Neuropsychopharmacology 2005;30:775-85.

33 Steinberg EE, Keiflin R, Boivin JR, et al. A causal link between prediction errors, dopamine neurons and learning. Nat Neurosci 2013;16:966-73.

34 Cox J, Witten IB. Striatal circuits for reward learning and decision-making. Nat Rev Neurosci 2019:20:482-94

35 Mohebi A, Pettibone JR, Hamid AA, et al. Dissociable dopamine dynamics for learning and motivation. Nature 2019;570:65-70.

36 Hamid AA, Frank MJ, Moore C. I. Dopamine waves as a mechanism for spatiotempora credit assignment. bioRxiv 2019.

37 Breitenstein C, Korsukewitz C, Flöel A, et al. Tonic dopaminergic stimulation impairs associative learning in healthy subjects. Neuropsychopharmacology 2006;31:2552-64.

38 Sharp ME, Foerde K, Daw ND, et al. Dopamine selectively remediates 'model-based' reward learning: a computational approach. Brain 2016;139:355-64.

39 NICE. Parkinson's disease in adults. In: Guidance and guidelines. NICE Guidel. NICE, 2017.

40 Troeung L, Egan SJ, Gasson N. A meta-analysis of randomised placebo-controlled treatment trials for depression and anxiety in Parkinson's disease. PLoS One 2013;8:e79510.

41 van der Velden RMJ, Broen MPG, Kuijf ML, et al. Frequency of mood and anxiety fluctuations in Parkinson's disease patients with motor fluctuations: a systematic review. Movement Disorders 2018:33:1521-7.

42 Dunlop K, Rizvi SJ, Kennedy SH, et al. Clinical, behavioral, and neural measures of reward processing correlate with escitalopram response in depression: a Canadian biomarker integration network in depression (CAN-BIND-1) report. Neuropsychopharmacology 2020;45:1390-7. 
Supplementary references for Table 1.

s1. den Brok, M. G. H. E. et al. Apathy in Parkinson's disease: A systematic review and meta-analysis. Mov. Disord. (2015). doi:10.1002/mds.26208

s2. Lemke, M. R. Anhedonia, Depression, and Motor Functioning in Parkinson's Disease During Treatment With Pramipexole. J. Neuropsychiatr. (2005). doi:10.1176/appi.neuropsych.17.2.214

s3. Husain, M. \& Roiser, J. P. Neuroscience of apathy and anhedonia: A transdiagnostic approach. Nature Reviews Neuroscience (2018). doi:10.1038/s41583-018-0029-9

s4. Reijnders, J. S. A. M., Ehrt, U., Weber, W. E. J., Aarsland, D. \& Leentjens, A. F. G. A systematic review of prevalence studies of depression in Parkinson's disease. Movement Disorders (2008). doi:10.1002/mds.21803

s5. Schrag, A., Ben-Shlomo, Y. \& Quinn, N. How common are complications of Parkinson's disease? J. Neurol. 249, 419-423 (2002).

s6. Halahakoon, D. C. et al. Reward-Processing Behavior in Depressed Participants Relative to Healthy Volunteers: A Systematic Review and Meta-analysis. JAMA Psychiatry (2020). doi:10.1001/jamapsychiatry.2020.2139

s7. Broen, M. P. G., Narayen, N. E., Kuijf, M. L., Dissanayaka, N. N. W. \& Leentjens, A. F. G. Prevalence of anxiety in Parkinson's disease: A systematic review and meta-analysis. Mov. Disord. 31, 1125-1133 (2016).

s8. $\quad$ Charpentier, C. J., Aylward, J., Roiser, J. P. \& Robinson, O. J. Enhanced Risk Aversion, But Not Loss Aversion, in Unmedicated Pathological Anxiety. Biol. Psychiatry 81, 1014-1022 (2017).

s9. Drew, D. S. et al. Dopamine and reward hypersensitivity in Parkinson's disease with I mpulse control disorder. Brain 143, 2502-2518 (2020).

s10.. Pezzella, F. R. et al. Prevalence and clinical features of hedonistic homeostatic dysregulation in Parkinson's disease. Mov. Disord. 20, 77-81 (2005).

s11. Luijten, M., Schellekens, A. F., Kühn, S., Machielse, M. W. J. \& Sescousse, G. Disruption of Reward Processing in Addiction: An Image-Based Meta-analysis of Functional Magnetic Resonance Imaging Studies. JAMA Psychiatry 74, 387-398 (2017).

s12. Fénelon, G. \& Alves, G. Epidemiology of psychosis in Parkinson's disease. J. Neurol. Sci. 289, 12-17 (2010).

S13. Radua, J. et al. Ventral Striatal Activation During Reward Processing in Psychosis: A Neurofunctional Meta-Analysis. JAMA Psychiatry 72, 1243-1251 (2015). 


\section{Supplement figure 1. Contour-Enhanced Funnel Plot of all studies Parkinson's versus healthy controls}

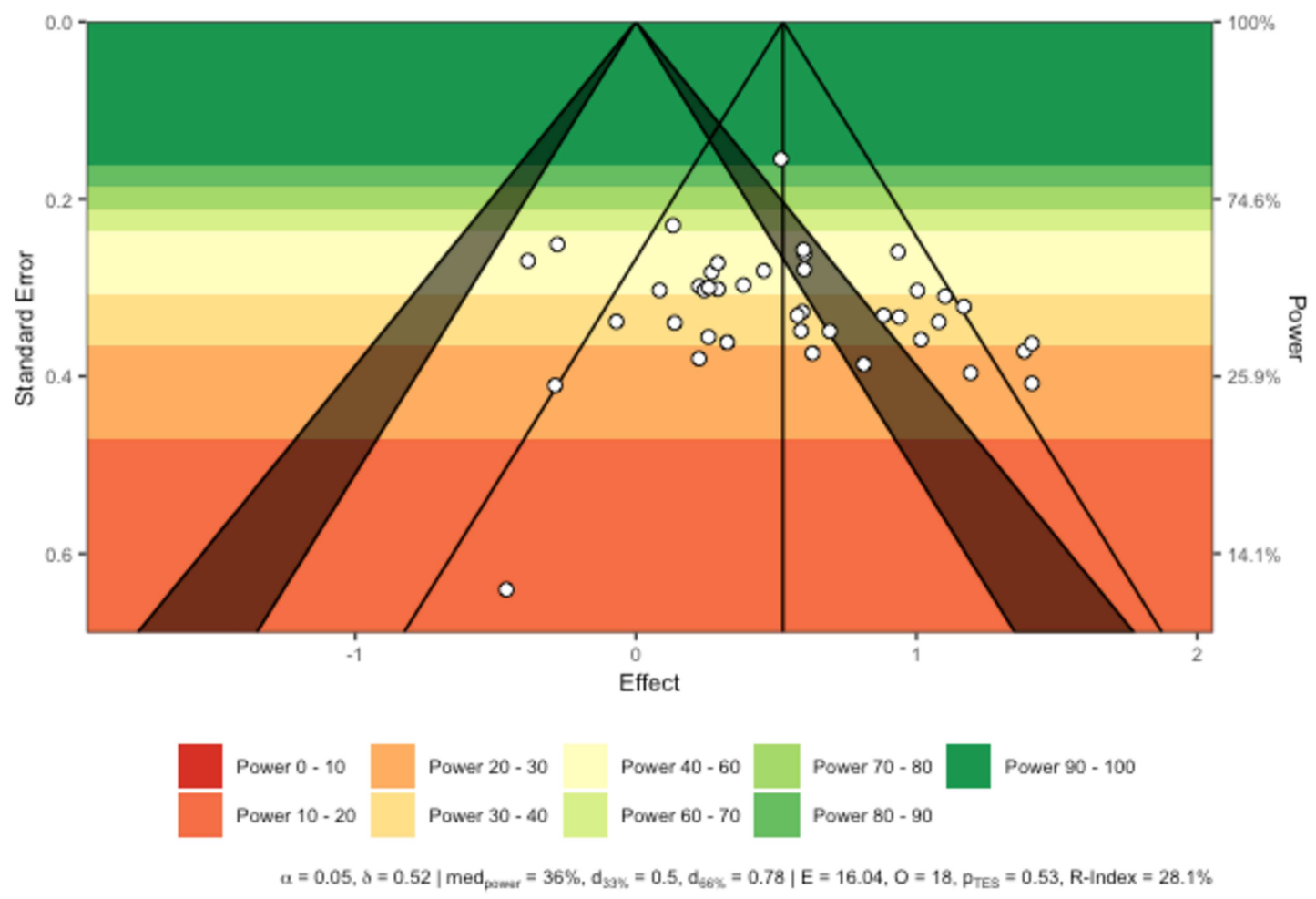

Supplement figure 2. Forest plot of reinforcement learning (RL) in Parkinson's patients with and without Impulse Control Disorder (ICD)

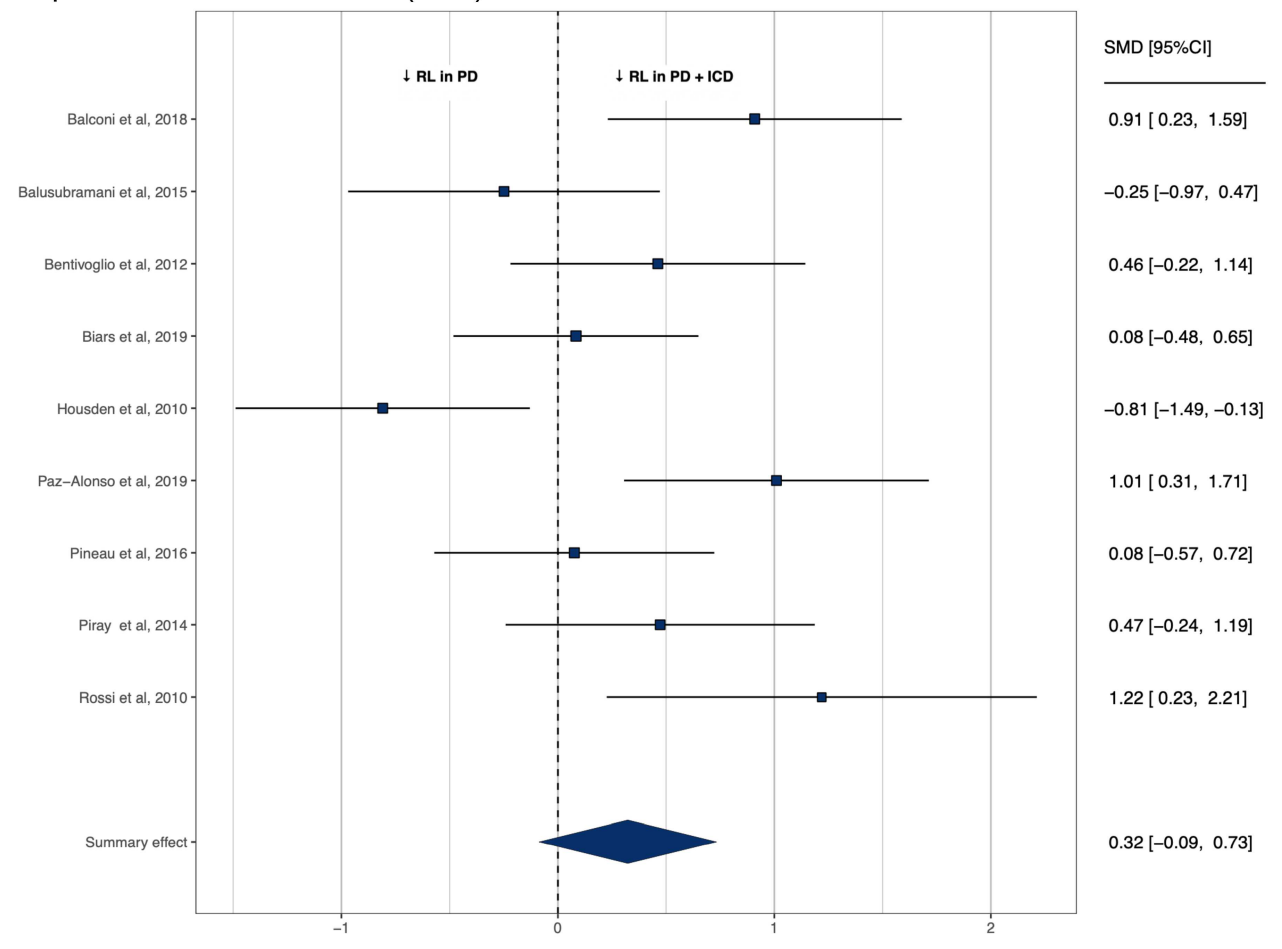




\section{Formulae used to convert study measures into Cohen's ds and associated variances.}

The following formulae were used to convert study measures into Cohen's ds and associated variances between subjects:

$$
d=\frac{M_{1}-M_{2}}{S D_{\text {pooled }}}
$$

Equation 1. Cohen's d from Means and Standard Deviations of 2 samples. $d$ is Cohen's d, M1 is the mean of one sample M2 is the mean of the other sample, SDpooled is the pooled standard deviation of the two samples (please see below.)

$$
S D_{\text {pooled }}=\sqrt{\left(N_{1}-1\right) S D_{1}^{2}+\left(N_{2}-1\right) S D_{2}^{2}}
$$

Equation 2. Pooled standard deviation of 2 samples. SDpooled is the pooled standard deviation of the two samples, N1 is the size of one sample N2 is the size of the other sample, SD1 is the standard deviation of one sample, SD2 is the standard deviation of the other sample.

$$
d=\frac{t}{\sqrt{\frac{1}{N_{1}}+\frac{1}{N_{2}}}}
$$

Equation 3. Cohen's d from t-statistic. $d$ is Cohen's d, N1 is the size of one sample N2 is the size of the other sample, $t$ is the tstatistic

$$
d=\frac{F}{\sqrt{\frac{1}{N_{1}}+\frac{1}{N_{2}}}}
$$

Equation 4. Cohen's d from F-statistic. $d$ is Cohen's d, N1 is the size of one sample N2 is the size of the other sample, F is the Fstatistic.

$$
\operatorname{Var}_{d}=\frac{N_{1}+N_{2}}{N_{1} \times N_{2}}+\frac{d^{2}}{2\left(N_{1}+N_{2}\right)}
$$

Equation 5. Variance on Cohen's d for between subjects. Vard is the Variance on Cohen's d, N1 is the size of one sample N2 is the size of the other sample, $d$ is Cohen's $d$.

The following formulae were used to convert study measures into Cohen's ds and associated variances within subjects:

$$
d=\frac{M_{d}}{S D_{d}}
$$

Equation 6. Cohen's d from Means and Standard Deviations from within subjects sample. Where $M_{d}$ is the mean change and $S D_{d}$ is the $\mathrm{SD}$ of the change scores

(equal to $S D_{d}=\sqrt{S D_{1}^{2}+S D_{2}^{2}-2 \times r \times S D_{1} S D_{2}}$ ).

$$
d=\frac{t}{\sqrt{N}}
$$

Equation 7. Cohen's d from t-statistic for within subjects results. $\mathrm{d}$ is Cohen's $\mathrm{d}, \mathrm{N}$ is the sample size, $\mathrm{t}$ is the t-statistic

$$
d=\sqrt{\frac{F}{N}}
$$

Equation 8. Cohen's d from F-statistic. $d$ is Cohen's d, $N$ is the sample size, $\mathrm{F}$ is the F-statistic.

$$
\operatorname{Var}[d]=\frac{1}{n}+\frac{d^{2}}{2 n}
$$

Equation 9. Variance on Cohen's d for within subjects, $\mathrm{n}$ is the size of the sample. 
Supplement table 1. Option Valuation study characteristics and participant demographics.

\begin{tabular}{|c|c|c|c|c|c|c|c|c|c|c|c|c|c|c|c|c|}
\hline \multicolumn{4}{|c|}{ Option valuation in Parkinson's vs Healthy Controls } & \multicolumn{2}{|c|}{ Age (years) } & \multirow[b]{2}{*}{$\begin{array}{c}\% \\
\text { female }\end{array}$} & \multicolumn{2}{|c|}{ Disease duration (years) } & \multicolumn{2}{|c|}{ UPDRS } & \multicolumn{3}{|c|}{ Cognition } & \multicolumn{3}{|c|}{ Depression } \\
\hline Study & Task & Group & $\mathbf{n}$ & Mean & SD & & Mean & SD & Mean & SD & $\begin{array}{l}\text { Cognitive } \\
\text { measure }\end{array}$ & Mean & SD & $\begin{array}{c}\text { Depression } \\
\text { measure }\end{array}$ & Mean & SD \\
\hline \multirow{2}{*}{$\begin{array}{l}\text { Bayard, } \\
2016\end{array}$} & \multirow{2}{*}{ Game of dice task } & PD & 78 & 67.49 & 8.16 & 35 & 7 & . & 24 & $(5-80)$ & MMSE & 28.1 & 1.93 & $\mathrm{BDI}$ & 12.5 & 7.38 \\
\hline & & $\mathrm{HC}$ & 96 & 67.95 & 6.75 & 32 & - & - & - & - & & 28.46 & 1.38 & & 10.04 & 11.76 \\
\hline \multirow{2}{*}{$\begin{array}{l}\text { Brandt, } \\
2015\end{array}$} & \multirow{2}{*}{ Game of dice task } & PD & 15 & 64.78 & 8.09 & 46.66 & . & . & 14.43 & 10.14 & MoCA & 26.87 & 1.69 & GDS-15 & 3.33 & 3.54 \\
\hline & & $\mathrm{HC}$ & 15 & 62.39 & 10.04 & 40 & - & - & - & - & & 27.6 & 1.43 & & 1.33 & 1.18 \\
\hline \multirow{2}{*}{$\begin{array}{l}\text { Chong, } \\
2015\end{array}$} & \multirow{2}{*}{$\begin{array}{l}\text { Effort based decision } \\
\text { making task }\end{array}$} & PD & 26 & 66.6 & 6.8 & 34.6 & . & . & 21.6 & 11.7 & MoCA & 28.2 & 1.3 & DASS & 2 & 2.23 \\
\hline & & $\mathrm{HC}$ & 26 & 66.2 & 6.4 & 42.3 & - & - & - & - & & 28.2 & 1.7 & & 1.5 & 1.84 \\
\hline \multirow{2}{*}{ Cools, 2003} & \multirow{2}{*}{$\begin{array}{l}\text { Decision making } \\
\text { gambling task }\end{array}$} & PD & 12 & 64.6 & 1.5 & 58.33 & 6.5 & 1.4 & 30.9 & 6.8 & MMSE & 29.5 & 0.15 & $\mathrm{BDI}$ & 7.7 & 1.1 \\
\hline & & $\mathrm{HC}$ & 12 & & . & & - & - & - & - & & & & & . & \\
\hline \multirow{2}{*}{$\begin{array}{l}\text { Kobayashi, } \\
2019\end{array}$} & \multirow{2}{*}{ Economic choice task } & $\mathrm{PD}$ & 15 & 63 & 6.58 & 40 & 6.93 & 6.2 & 39 & 14.6 & MMSE & 28.3 & 1.76 & . & . & . \\
\hline & & $\mathrm{HC}$ & 21 & 60 & 6.69 & 57.1 & - & - & - & - & & 28.7 & 1.99 & & . & . \\
\hline \multirow{2}{*}{$\begin{array}{l}\text { Le Bouc, } \\
2016\end{array}$} & \multirow{2}{*}{$\begin{array}{l}\text { Incentivised grip } \\
\text { force choice task }\end{array}$} & $\mathrm{PD}$ & 24 & 60.2 & 1.6 & 29.17 & 11.4 & 1.3 & 11.7 & 1.7 & MMSE & 27.3 & 1.3 & MADRS & 5.9 & 1 \\
\hline & & $\mathrm{HC}$ & 25 & 57 & 2.1 & 52 & - & - & - & - & & . & . & & 2.9 & 0.7 \\
\hline \multirow{2}{*}{$\begin{array}{l}\text { Le Heron, } \\
2018\end{array}$} & \multirow{2}{*}{$\begin{array}{l}\text { Effort based decision } \\
\text { making task }\end{array}$} & $\mathrm{PD}$ & 39 & 67.8 & 7.6 & 19.04 & . & . & 29.5 & 9.9 & ACE & 91.1 & 7.9 & $\mathrm{BDI}$ & 14.3 & 7.7 \\
\hline & & $\mathrm{HC}$ & 32 & 68.9 & 6.9 & 40.6 & - & - & - & - & & 95.6 & 3.8 & & 3.8 & 3.7 \\
\hline \multirow{2}{*}{$\begin{array}{l}\text { Mcguigan, } \\
2018\end{array}$} & \multirow{2}{*}{ Cognitive effort task } & PD & 20 & 67.1 & 9.1 & 40 & 5.4 & 4.9 & 27.3 & 18.1 & MoCA & 27.7 & 1.92 & $\mathrm{BDI}$ & 9.5 & 4.98 \\
\hline & & $\mathrm{HC}$ & 20 & 61.1 & 13.6 & 40 & - & - & - & - & & 28.1 & 1.37 & & 3.55 & 4.13 \\
\hline \multirow{2}{*}{ Torta, 2009} & \multirow{2}{*}{$\begin{array}{l}\text { Cambridge gamble } \\
\text { task }\end{array}$} & PD & 15 & 58.4 & 6.9 & 13.33 & 13.2 & 3.2 & 16.52 & 7.5 & MMSE & 28.2 & 1.5 & $\mathrm{BDI}$ & 10 & 6.6 \\
\hline & & $\mathrm{HC}$ & 13 & 58.2 & 5.7 & 15.38 & - & - & - & - & & & . & & . & . \\
\hline \multirow{2}{*}{$\begin{array}{l}\text { Sharp, } \\
2013\end{array}$} & Vancouver gambling & $\mathrm{PD}$ & 18 & 65.47 & 9.17 & 27.78 & 5.59 & 4.04 & 20.65 & 6.91 & MOCA & 27.94 & 1.14 & $\mathrm{BDI}$ & 6.06 & 3.78 \\
\hline & task (modified) & $\mathrm{HC}$ & 18 & 66.76 & 5.83 & 50 & - & - & - & & & 28.85 & 1.32 & & 5.18 & 3.57 \\
\hline Option va & $\begin{array}{l}\text { ation in Parkinson's wi } \\
\text { syndrome vs without }\end{array}$ & psychiat & & Age ( & ears) & & Disease dur & & UP & & & gnition & & Dep & ression & \\
\hline Study & Task & Group & $\mathrm{n}$ & Mean & SD & $\begin{array}{c}\% \\
\text { female }\end{array}$ & Mean & SD & Mean & SD & $\begin{array}{l}\text { Cognitive } \\
\text { measure }\end{array}$ & Mean & SD & $\begin{array}{c}\text { Depression } \\
\text { measure }\end{array}$ & Mean & SD \\
\hline Haagensen, & Game of dice task & $\mathrm{PD}$ & 13 & 61.4 & 9.7 & 46.15 & 4.5 & 2 & 22.8 & 6.9 & MoCA & 28.7 & 1.3 & $\mathrm{BDI}$ & 7 & 5 \\
\hline 2020 & & $\mathrm{ICD}$ & 13 & 59.4 & 10.9 & 38.46 & 6.5 & 3.6 & 22.6 & 6 & & 28.7 & 0.9 & & 7.4 & 6.5 \\
\hline Kobayashi, & Eronomic choire tack & $\mathrm{PD}$ & 15 & 63 & 6.58 & 40 & 6.93 & 6.2 & 39 & 14.6 & MMSE & 28.3 & 1.76 & . & . & \\
\hline 2019 & 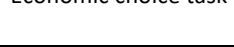 & $I C D$ & 10 & 63.1 & 9.68 & 40 & 8.4 & 2.99 & 49.9 & 26.3 & & 28.3 & 1.77 & $\cdot$ & . & $\cdot$ \\
\hline Le Heron, & Effort based decision & $\mathrm{PD}$ & 18 & 68.2 & 6.5 & 44.44 & . & . & 27.1 & 13.2 & ACE & 93.5 & 5 & $\mathrm{BDI}$ & 11 & 7 \\
\hline 2018 & & Apathy & 21 & 67.5 & 8.5 & 19.05 & & & 29.5 & 9.9 & & 89.4 & 9.4 & & 17.1 & 7.4 \\
\hline
\end{tabular}


\begin{tabular}{|l|l|c|c|c|c|c|}
\multirow{3}{*}{ Voon, 2011 } & \multirow{3}{*}{ Gambling task } & PD & 14 & 54.5 & 12.5 & 71.4 \\
\cline { 2 - 7 } & & ICD & 14 & 51.5 & 8.3 & 71.4 \\
\hline
\end{tabular}

$P D=$ Parkinson's disease, $H C=$ healthy controls, ICD = Impulse control disorder, $S D=$ standard deviation, IQR = interquartile range, ' $. '=$ not reported, ' - ' = not applicable, UPDRS = Unified Parkinson's Disease Rating Scale, MMSE = Mini-mental state examination, MoCA = Montreal cognitive assessment, ACE = Addenbrooke's cognitive examination, BDI $=$ Beck depression inventory, DASS $=$ Depression, Anxiety and Stress Scale, GDS = Geriatric depression scale, MADRS = Montgomery-Asberg Depression Rating Scale

Supplement table 2. Reinforcement Learning study characteristics and participant demographics.

\begin{tabular}{|c|c|c|c|c|c|c|c|c|c|c|c|c|c|c|c|c|}
\hline \multicolumn{4}{|c|}{ Reinforcement learning in Parkinson's vs Healthy Controls } & \multicolumn{2}{|c|}{ Age (years) } & \multirow[b]{2}{*}{$\begin{array}{c}\% \\
\text { female }\end{array}$} & \multicolumn{2}{|c|}{ Disease duration (years) } & \multicolumn{2}{|c|}{ UPDRS } & \multicolumn{3}{|c|}{ Cognition } & \multicolumn{3}{|c|}{ Depression } \\
\hline Study & Task & Group & $\mathrm{n}$ & Mean & $\begin{array}{l}\text { SD or } \\
\text { (IQR) }\end{array}$ & & Mean & $\begin{array}{l}\text { SD or } \\
\text { (IQR) }\end{array}$ & Mean & $\begin{array}{l}\text { SD or } \\
\text { (IQR) }\end{array}$ & Measure & Mean & $\begin{array}{l}\text { SD or } \\
\text { (IQR) }\end{array}$ & Measure & Mean & $\begin{array}{l}\text { SD or } \\
\text { (IQR) }\end{array}$ \\
\hline \multirow{2}{*}{ Castrioto, 2015} & \multirow{2}{*}{ IGT } & PD & 20 & 53.2 & 6.6 & 45 & 10.3 & 3.8 & 12.3 & 6.1 & MDRS & 137.6 & 4.2 & . & . & . \\
\hline & & $\mathrm{HC}$ & 24 & 54.9 & 7.6 & 62.5 & - & - & - & - & & 140.7 & 2.4 & & . & . \\
\hline \multirow{2}{*}{$\begin{array}{l}\text { Balusubramani, } \\
\quad 2015\end{array}$} & \multirow{2}{*}{$\begin{array}{c}\text { Probabilistic } \\
\text { rewarded } \\
\text { categorisation } \\
\text { learning task }\end{array}$} & PD & 30 & . & . & 18.75 & 9.5 & . & . & . & MMSE & . & . & $\mathrm{BDI}$ & . & . \\
\hline & & $\mathrm{HC}$ & 20 & & . & 13.04 & - & - & - & - & & & & & 5 & 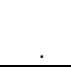 \\
\hline \multirow{2}{*}{ Bodi, 2009} & \multirow{2}{*}{$\begin{array}{c}\text { Probabilistic } \\
\text { classification task }\end{array}$} & PD & 22 & 44.8 & 5.2 & 30.77 & 0.266 & 0.166 & 27.5 & 6.1 & . & . & . & HAM-D & 4.2 & 1.4 \\
\hline & & $\mathrm{HC}$ & 20 & 45.3 & 8.5 & 25 & - & - & - & - & & . & . & & . & . \\
\hline \multirow{2}{*}{ Buelow, 2014} & \multirow{2}{*}{ IGT } & $\mathrm{PD}$ & 24 & 68.04 & 7.86 & 45.8 & . & . & 29.89 & 13.14 & MMSE & 28.33 & 1.63 & GDS & 2.5 & 1.91 \\
\hline & & $\mathrm{HC}$ & 14 & 69.62 & 6.36 & 53.85 & - & - & - & - & & 29.31 & 1.11 & & 1.15 & 1.57 \\
\hline \multirow{2}{*}{$\begin{array}{c}\text { Cavanagh, } \\
2017\end{array}$} & \multirow{2}{*}{ Cost of conflict task } & $\mathrm{PD}$ & 28 & 69.75 & 8.59 & 39.28 & 5.54 & 4.18 & & & MMSE & 28.64 & 1.06 & $\mathrm{BDI}$ & 7.64 & 5.23 \\
\hline & & $\mathrm{HC}$ & 28 & 69.21 & 9.23 & 39.28 & - & - & - & - & & 28.82 & 1.02 & & 4.93 & 4.69 \\
\hline \multirow{2}{*}{$\begin{array}{l}\text { Czernecki, } \\
2002\end{array}$} & \multirow{2}{*}{ IGT } & PD & 23 & 57.6 & 2.1 & 60.87 & 14.9 & 1.2 & 12.4 & 2 & MDRS & 139.1 & 0.8 & MADRS & 8.3 & 1.4 \\
\hline & & $\mathrm{HC}$ & 28 & 58.1 & 1.7 & 35.71 & - & - & - & - & & 141.1 & 0.3 & & 6.2 & 0.8 \\
\hline \multirow{2}{*}{ Delazer, 2009} & \multirow{2}{*}{ IGT } & $\mathrm{PD}$ & 20 & 68.5 & 5.9 & 25 & 5.25 & 6.38 & 17.6 & 8.7 & MMSE & 27.8 & 1.9 & HADS & 6.6 & 3.2 \\
\hline & & $\mathrm{HC}$ & 20 & 71.3 & 3.5 & 85 & - & - & - & - & & 29.8 & 0.4 & & . & . \\
\hline \multirow{2}{*}{$\begin{array}{l}\text { Euteneuer, } \\
2009\end{array}$} & \multirow{2}{*}{ IGT } & PD & 21 & 67.6 & 7.31 & 67 & 7.14 & 6.06 & 17.7 & 9.2 & MMSE & 29 & 1.1 & $\mathrm{BDI}$ & 3.9 & 2.12 \\
\hline & & $\mathrm{HC}$ & 23 & 64.4 & 8.56 & 48 & & & & & & 29.65 & 0.65 & & 0.83 & 1.3 \\
\hline \multirow{2}{*}{ Evens, 2015} & \multirow{2}{*}{ IGT } & PD & 32 & 65.12 & 8.25 & 25 & 7.38 & 4.33 & 16.58 & 7.36 & MMSE & 29.25 & 0.98 & MADRS & 4.33 & 3.68 \\
\hline & & $\mathrm{HC}$ & 32 & 65.53 & 5.94 & 31 & - & - & - & - & & 29.34 & 0.83 & & 1.59 & 2.19 \\
\hline \multirow{2}{*}{ Garofalo, 2017} & \multirow{2}{*}{$\begin{array}{c}\text { Instrumental } \\
\text { conditioning task }\end{array}$} & PD & 17 & 63.29 & 9.94 & 50 & 16.42 & 28.77 & . & . & MMSE & 28.41 & 1.37 & $\mathrm{BDI}$ & 12.66 & 7.83 \\
\hline & & $\mathrm{HC}$ & 24 & 61.91 & 5.83 & 44 & - & - & - & - & & 28.94 & 1.54 & & 8.88 & 4.94 \\
\hline \multirow{2}{*}{$\begin{array}{l}\text { Gescheidt, } \\
2012\end{array}$} & IGT & PD & 19 (early onset) & 50.32 & 8.74 & 25 & 11.32 & 6.42 & 14.6 & 8.7 & MMSE & 29.37 & 0.96 & MADRS & . & . \\
\hline & & $\mathrm{HC}$ & 20 & 49.95 & 9.03 & 26.3 & - & - & - & - & & 29.7 & 0.47 & & . & . \\
\hline Graef, 2010 & & PD & 15 & 65.27 & 8.14 & 40 & 4.3 & 4 & 19.64 & 7.7 & MMSE & 29 & 1 & $\mathrm{BDI}$ & 6.93 & 5.55 \\
\hline
\end{tabular}




\begin{tabular}{|c|c|c|c|c|c|c|c|c|c|c|c|c|c|c|c|c|}
\hline & $\begin{array}{c}\text { Probabilistic } \\
\text { reversal learning } \\
\text { task }\end{array}$ & $\mathrm{HC}$ & 16 & 67.75 & 4.55 & 37.5 & - & - & - & - & & 28.64 & 1.15 & & 5.31 & 2.98 \\
\hline \multirow{2}{*}{ Herzallah, 2017} & \multirow{2}{*}{$\begin{array}{l}\text { Rewarded } \\
\text { categorisation task }\end{array}$} & PD & 17 & 59.4 & 12.6 & 11.76 & 5.24 & 4 & 28.6 & 14.6 & MMSE & 28.5 & 1.1 & $\mathrm{BDI}$ & 8.3 & 5.2 \\
\hline & & $\mathrm{HC}$ & 15 & 54.3 & 12.3 & 33.33 & - & - & - & - & & 29.3 & 0.7 & & 6.7 & 5.3 \\
\hline \multirow{2}{*}{ Housden, 2010} & \multirow{2}{*}{$\begin{array}{l}\text { Rewarded salience } \\
\text { attribution test }\end{array}$} & PD & 18 & 67.7 & 5.5 & 38.89 & 12.9 & 8.3 & 20 & 6.6 & MMSE & 28.6 & 2.1 & $\mathrm{BDI}$ & 12.9 & 9.9 \\
\hline & & $\mathrm{HC}$ & 20 & 65.5 & 6 & 33.33 & - & - & - & - & & 29.4 & 0.8 & & 11.3 & 6.9 \\
\hline \multirow{2}{*}{$\begin{array}{l}\text { Ibarretxe- } \\
\text { Bilbao, } 2009\end{array}$} & \multirow{2}{*}{ IGT } & PD & 24 & 56.13 & 8.5 & 33 & 3.06 & 1.6 & 14.67 & 3.5 & MMSE & 29.63 & 0.5 & $\mathrm{BDI}$ & $\cdot$ & . \\
\hline & & $\mathrm{HC}$ & 24 & 57.58 & 8.9 & 33 & - & - & - & - & & 29.83 & 0.4 & & & \\
\hline \multirow{2}{*}{$\begin{array}{l}\text { Kobayakawa, } \\
2008\end{array}$} & \multirow{2}{*}{ IGT } & $\mathrm{PD}$ & 34 & 69.9 & 8.9 & 64.7 & 6.4 & 3.4 & . & . & MMSE & 28 & 2.2 & SDS & 36.1 & 8.9 \\
\hline & & $\mathrm{HC}$ & 22 & 67.6 & 6.9 & 40.9 & - & - & - & - & & 28.8 & 1.6 & & 29.1 & 6 \\
\hline \multirow{2}{*}{$\begin{array}{l}\text { Kobayakawa, } \\
2010\end{array}$} & \multirow{2}{*}{ IGT } & $\mathrm{PD}$ & 14 & 68.9 & 8 & 50 & 5.6 & 2.7 & . & . & MMSE & 28.2 & 1.9 & SDS & 32.9 & 7.8 \\
\hline & & $\mathrm{HC}$ & 22 & 67.6 & 6.9 & 69.2 & - & - & - & - & & 28.8 & 1.6 & & 29.1 & 6 \\
\hline \multirow{2}{*}{ Mapelli, 2014} & \multirow{2}{*}{ IGT } & $\mathrm{PD}$ & 15 & 61.4 & 9.6 & 25 & 4.8 & 3.4 & 8.9 & 4 & MMSE & 28.3 & 1.2 & $\mathrm{BDI}$ & \multicolumn{2}{|c|}{ Excluded if $>14$} \\
\hline & & $\mathrm{HC}$ & 15 & 60.7 & 9.8 & 26.7 & - & - & - & - & & 27.86 & 1.5 & & . & . \\
\hline \multirow{2}{*}{ Mimura, 2006} & \multirow{2}{*}{ IGT } & $\mathrm{PD}$ & 18 & 68.9 & 7 & 72.2 & . & . & . & . & MMSE & 27.8 & 1.9 & ZSRD & 39.4 & 6.9 \\
\hline & & $\mathrm{HC}$ & 20 & . & . & 70 & - & - & - & - & & 29.1 & 1.5 & & 30.5 & 6 \\
\hline \multirow{2}{*}{$\begin{array}{c}\text { Pagonabarraga, } \\
2007\end{array}$} & \multirow{2}{*}{ IGT } & PD & 35 & 67.2 & 8 & 37.2 & 8.4 & 5 & 21.2 & 8 & MDRS & 133 & 6 & & . & . \\
\hline & & $\mathrm{HC}$ & 31 & 70.2 & 10 & 47.7 & - & - & - & - & & 136 & 5 & & . & . \\
\hline \multirow{2}{*}{$\begin{array}{l}\text { Paz-Alonso, } \\
2019\end{array}$} & \multirow{2}{*}{ IGT } & $\mathrm{PD}$ & 17 & 61 & 8.7 & 11.8 & 7 & $(4-10)$ & 25.9 & 8.2 & . & & & HADS-D & 2.2 & 2.6 \\
\hline & & $\mathrm{HC}$ & 18 & 63 & 9.7 & 16.7 & - & - & - & - & & & & & 1.9 & 1.3 \\
\hline \multirow{3}{*}{ Perretta, 2005} & \multirow{3}{*}{ IGT } & PD (late stage) & 16 & 77.7 & 6 & 50 & . & . & 27.2 & 1.3 & MMSE & Excluded if $<27$ & . & $\mathrm{BDI}$ & 12.7 & 1.5 \\
\hline & & PD (early stage) & 16 & 72.4 & 2.3 & 43.75 & . & & 11.3 & 1.1 & & & & & 6.9 & 0.7 \\
\hline & & $\mathrm{HC}$ & 19 & 72.6 & 8.28 & 42.1 & - & - & - & - & & & & & 5.2 & 0.8 \\
\hline \multirow{2}{*}{ Pignatti, 2012} & \multirow{2}{*}{ IGT } & $\mathrm{PD}$ & 15 & 64.27 & 10.5 & . & . & . & . & . & MMSE & 28.31 & 1.11 & . & . & . \\
\hline & & $\mathrm{HC}$ & 16 & 41.56 & 13.9 & . & - & - & - & - & & & & & . & . \\
\hline \multirow{2}{*}{ Piray, 2014} & \multirow{2}{*}{$\begin{array}{c}\text { Rewarded } \\
\text { categorisation task }\end{array}$} & $\mathrm{PD}$ & 40 & 63.33 & 3.98 & 23.07 & 9.72 & 2.64 & 19.6 & 6.42 & MMSE & 27 & 0.93 & $\mathrm{BDI}$ & 8 & 1.69 \\
\hline & & $\mathrm{HC}$ & 20 & 66.45 & 4.7 & 35 & - & - & - & - & & 27.65 & 1.18 & & 7.75 & 1.97 \\
\hline Poletti, 2010 & IGT & PD (off) & 24 & 64.9 & 5.8 & 27 & 0 (de novo) & & 14.3 & 8.1 & MMSE & 28.8 & 2 & GDS & 4.7 & 3.3 \\
\hline & & $\mathrm{HC}$ & 25 & 65.4 & 2.2 & 44 & & & & & & 28.5 & 1.8 & & 2.8 & 1.4 \\
\hline Thiel 2003 & JGT & $\mathrm{PD}$ & 5 & 62.6 & 12.5 & 40 & 8 & 2.55 & 20.6 & 6.47 & MMSE & .28 .4 & 2.07 & & . & . \\
\hline & 然 & $\mathrm{HC}$ & 5 & 44.2 & 21.3 & 20 & - & - & - & - & & & & & & \\
\hline $\mathrm{Xi}, 2015$ & IGT & PD & 15 & 60.73 & 11.7 & 53 & 4.33 & 5.05 & 15.87 & 8.96 & MMSE & 27.6 & 4.63 & HAMD & 4.67 & 1.29 \\
\hline
\end{tabular}




\begin{tabular}{|c|c|c|c|c|c|c|c|c|c|c|c|c|c|c|c|c|}
\hline & & $\mathrm{HC}$ & 15 & 56.33 & 14.5 & 60 & - & - & - & - & & 29.4 & 1.12 & & 3.8 & 1.37 \\
\hline \multirow{2}{*}{ Yildirim, 2020} & \multirow{2}{*}{ IGT } & PD & 39 & 65.9 & 7.3 & 21 & 7.8 & 4.3 & 11.6 & 6.1 & MMSE & . & . & . & . & . \\
\hline & & $\mathrm{HC}$ & 37 & 64.4 & 8 & 24 & - & - & - & - & & & . & & . & . \\
\hline \multicolumn{4}{|c|}{$\begin{array}{l}\text { Reinforcement learning in Parkinson's with psychiatric syndrome vs } \\
\text { without }\end{array}$} & \multicolumn{2}{|c|}{ Age } & & \multicolumn{2}{|c|}{ Disease duration (years) } & \multicolumn{2}{|c|}{ UPDRS } & \multicolumn{3}{|c|}{ Cognition } & & \multicolumn{2}{|c|}{ Depression } \\
\hline Study & Task & Group & $\mathrm{n}$ & Mean & $\begin{array}{l}\text { SD or } \\
\text { (IQR) }\end{array}$ & $\begin{array}{c}\% \\
\text { female }\end{array}$ & Mean & $\begin{array}{l}\text { SD or } \\
\text { (IQR) }\end{array}$ & Mean & $\begin{array}{l}\text { SD or } \\
\text { (IQR) }\end{array}$ & Measure & Mean & $\begin{array}{l}\text { SD or } \\
\text { (IQR) }\end{array}$ & $\begin{array}{c}\text { Depression } \\
\text { measure }\end{array}$ & Mean & $\begin{array}{l}\text { SD or } \\
\text { (IQR) }\end{array}$ \\
\hline \multirow{2}{*}{ Balconi, 2018} & \multirow{2}{*}{ IGT } & PD & 20 & 63.9 & 7.1 & 15 & & & 13 & 9.3 & & & . & $\mathrm{BDI}$ & 11.3 & 6 \\
\hline & & PG & 17 & 60.7 & 6.1 & 17.65 & . & & 17 & 7.8 & & . & . & & 15.9 & 9.1 \\
\hline \multirow{2}{*}{$\begin{array}{l}\text { Balusubramani, } \\
\quad 2015\end{array}$} & \multirow{2}{*}{$\begin{array}{l}\text { Rewarded } \\
\text { categorisation task }\end{array}$} & PD & 14 & . & . & 21.4 & 8.35 & & . & . & MMSE & . & . & $\mathrm{BDI}$ & . & . \\
\hline & & $\mathrm{ICD}$ & 16 & 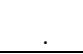 & & 12.5 & 9.56 & & & & & & & & & \\
\hline \multirow{2}{*}{$\begin{array}{l}\text { Bentivoglio, } \\
2012\end{array}$} & \multirow{2}{*}{ IGT } & PD & 17 & 63.94 & 9.2 & 35.29 & 7.3 & 4.4 & 22.5 & 6.9 & MMSE & 28.6 & 1.4 & HAM-D & 5.6 & 4.1 \\
\hline & & ICD & 17 & 62 & 10.1 & 17.65 & 6.9 & 3.8 & 23.8 & 11 & & 28.4 & 1.6 & & 7.5 & 6.3 \\
\hline \multirow{2}{*}{ Biars, 2019} & \multirow{2}{*}{ IGT } & $\mathrm{PD}$ & 24 & 60.5 & 7.8 & 12.5 & 11.9 & 7.1 & 37.2 & 14.8 & DRS & 136.5 & 5.9 & $\mathrm{BDI}$ & 8.5 & 5.3 \\
\hline & & $\mathrm{ICD}$ & 24 & 61.2 & 8.3 & 12.5 & 13.2 & 7.1 & 21.6 & 9.9 & & 138 & 3.5 & & 11.2 & 6.8 \\
\hline \multirow{2}{*}{ Buelow, 2014} & \multirow{2}{*}{ IGT } & PD & 14 & 69 & 6.19 & 57.14 & . & & 29.62 & 6.85 & MMSE & 28.71 & 1.38 & GDS & 2.14 & 1.41 \\
\hline & & Apathy & 10 & 66.7 & 9.96 & 30 & . & & 29.89 & 13.14 & & 27.8 & 1.87 & & 3 & 2.45 \\
\hline \multirow{2}{*}{ Garofalo, 2017} & \multirow{2}{*}{$\begin{array}{l}\text { Instrumental } \\
\text { conditioning task }\end{array}$} & PD & 17 & 63.29 & 9.94 & 44 & 10.94 & 10.38 & . & . & MMSE & 28.94 & 1.54 & $\mathrm{BDI}$ & 8.88 & 4.94 \\
\hline & & Psychosis & 12 & 60.83 & 6.6 & 50 & 16.42 & 28.77 & . & . & & 28.41 & 1.37 & & 12.66 & 7.83 \\
\hline \multirow{2}{*}{ Herzallah, 2017} & \multirow{2}{*}{$\begin{array}{c}\text { Rewarded } \\
\text { categorisation task }\end{array}$} & PD & 17 & 59.4 & 12.6 & 11.76 & 5.24 & 4 & . & . & MMSE & 28.5 & 1.1 & $\mathrm{BDI}$ & 8.3 & 5.2 \\
\hline & & $M D D$ & 13 & 55.2 & 11.9 & 69.2 & 4.84 & 3.7 & 28.6 & 14.6 & & 27.5 & 1.8 & & 26.9 & 7.7 \\
\hline \multirow{2}{*}{ Housden, 2010} & \multirow{2}{*}{$\begin{array}{c}\text { Rewarded salience } \\
\text { attribution test }\end{array}$} & PD & 18 & 67.7 & 5.5 & 33.33 & 12.9 & 8.3 & 21.3 & 0.4 & MMSE & 29.4 & 0.8 & $\mathrm{BDI}$ & 11.3 & 6.9 \\
\hline & & $I C D$ & 18 & 62.3 & 7.6 & 38.89 & 13.9 & 9 & 20 & 6.6 & & 28.6 & 2.1 & & 12.9 & 9.9 \\
\hline \multirow{2}{*}{$\begin{array}{l}\text { Martinez- } \\
\text { Horta, } 2013\end{array}$} & ITT & PD & 17 & 65.06 & 4.85 & . & 5.38 & 4.25 & 18 & 4.61 & MMSE & 28.94 & 1.24 & HADS & 3.88 & 3.6 \\
\hline & | & Apathy & 17 & 68.25 & 6.06 & . & 6.75 & 4.93 & 18.65 & 5.8 & & 28.55 & 1.23 & & 5.65 & 2.92 \\
\hline Paz-Alonso, & IGT & PD & 17 & 61 & 8.7 & 11.8 & 7 & $(4-10)$ & 25.9 & 8.2 & . & . & . & HADS & 2.2 & 2.6 \\
\hline 2019 & & $\mathrm{ICD}$ & 18 & 62.3 & 7.6 & 11.1 & 8 & $(5.1-10)$ & 22.31 & 6.6 & & . & . & & 3.1 & 2.4 \\
\hline Pineau 2016 & IGT (adanted) & PD & 20 & 55 & $(40-62)$ & 35 & 5.5 & $(4-12)$ & 8.5 & $(0-34)$ & MDRS & 139 & (131-143) & . & . & \\
\hline & & ICD & 17 & 55 & $(37-65)$ & 17.64 & 7 & $(2-10)$ & 7 & $(0-23)$ & & 140 & $(133-144)$ & & . & \\
\hline Piray 2014 & Rewarded & $\mathrm{PD}$ & 40 & 63.33 & 3.98 & 25.9 & 8.87 & 3.14 & 19.6 & 6.42 & MMSE & 27 & 0.93 & $\mathrm{BDI}$ & 8 & 1.69 \\
\hline & categorisation task & $I C D$ & 16 & 64.38 & 3.32 & 11.1 & 9.63 & 2.45 & 19 & 5.32 & & 27.19 & 1.11 & & 6.75 & 1.69 \\
\hline Dolotti 201 & GT & PD & 12 & 63.92 & 7.17 & 16.66 & $\cdot$ & & . & . & MMSE & 28.72 & 2.41 & GDS-15 & 3.33 & 2.34 \\
\hline & & Alexithymia & 12 & 66.17 & 5.2 & 41.66 & . & & . & . & & 28.49 & 1.94 & & 6.33 & 3.89 \\
\hline Rossi, 2010 & IGT & PD & 13 & 65.1 & 3.8 & 23 & & & 14.7 & 6.7 & MMSE & & . & MADRS & 14.1 & 7.9 \\
\hline
\end{tabular}




\begin{tabular}{|c|c|c|c|c|c|c|c|c|c|c|c|c|c|c|c|c|}
\hline & & PG & 7 & 61.4 & 6.9 & 14 & & . & 17 & 9.1 & & Excluded if $<24$ & . & & 17.1 & 6.5 \\
\hline \multirow{2}{*}{$\begin{array}{l}\text { Sáez-Francàs, } \\
2014\end{array}$} & \multirow{2}{*}{ IGT } & PD & 56 & 62.64 & 9.06 & 32.14 & 4.31 & 3.65 & 18.41 & 5.82 & MMSE & . & . & HAM-D & 3.64 & 3.37 \\
\hline & & Fatigue & 33 & 61.73 & 9.85 & 39.4 & 4.94 & 3.44 & 20.85 & 6.31 & & Excluded if $<26$ & . & & 8.64 & 7.06 \\
\hline \multirow{2}{*}{ Timmer, 2017} & \multirow{2}{*}{$\begin{array}{l}\text { Rewarded task- } \\
\text { switching paradigm }\end{array}$} & $\mathrm{PD}$ & 22 & 61.1 & 7.6 & 36.4 & & & 21.9 & 6.8 & MMSE & 28.6 & 1.2 & $\mathrm{BDI}$ & 4.3 & 2.3 \\
\hline & & MDD & 19 & 58.4 & 5.3 & 36.8 & & & & & & 28.5 & 1.3 & & 8.7 & 5 \\
\hline
\end{tabular}

PD = Parkinson's disease, HC = healthy controls, IGT = lowa Gambling Task, ICD = Impulse control disorder, MDD = Major depressive disorder, SD = standard deviation, IQR = interquartile range, ' '.' = not reported, '-' = not applicable, UPDRS = Unified Parkinson's Disease Rating Scale, MMSE = Mini-mental state examination, MoCA = Montreal cognitive assessment, MDRS = Mattis dementia rating scale, BDI = Beck depression inventory, HAM-D = Hamilton

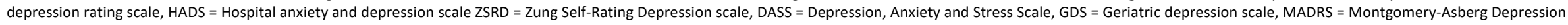
Rating Scale, SDS = Self-rated depression scale

Supplement table 3. Response Vigor and Reward Bias study characteristics and participant demographics.

\begin{tabular}{|c|c|c|c|c|c|c|c|c|c|c|c|c|c|c|c|c|}
\hline \multicolumn{4}{|c|}{ Response vigor in Parkinson's vs Healthy Controls } & \multicolumn{2}{|c|}{ Age } & \multirow[b]{2}{*}{$\begin{array}{c}\% \\
\text { female }\end{array}$} & \multicolumn{2}{|c|}{ Disease duration } & \multicolumn{2}{|c|}{ UPDRS } & \multicolumn{3}{|c|}{ Cognition } & \multicolumn{3}{|c|}{ Depression } \\
\hline Study & Task & Group & $\mathrm{n}$ & Mean & SD & & Mean & SD & Mean & SD & $\begin{array}{l}\text { Cognitive } \\
\text { measure }\end{array}$ & Mean & SD & $\begin{array}{c}\text { Depression } \\
\text { measure }\end{array}$ & Mean & SD \\
\hline \multirow{2}{*}{ Muhammed, 2016} & \multirow{2}{*}{$\begin{array}{l}\text { Speed of gaze } \\
\text { shifting task }\end{array}$} & PD & 16 & 67.3 & 6.4 & & 3.2 & 2.2 & 19.4 & 9.8 & MoCA & 27.8 & 2.3 & BDI & 11.7 & 5.5 \\
\hline & & $\mathrm{HC}$ & 31 & 65.9 & 5.6 & & - & - & - & - & & 28.2 & 1.6 & & 15.2 & 7.8 \\
\hline \multirow{2}{*}{ Renfroe, 2016} & \multirow{2}{*}{$\begin{array}{l}\text { Rewarded speed of } \\
\text { response to specific } \\
\text { visual stimuli }\end{array}$} & PD & 18 & 66 & 7.99 & 22.2 & 8.39 & 4.57 & 26 & 9.55 & & 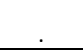 & . & $\mathrm{BDI}$ & 7.82 & 4.85 \\
\hline & & $\mathrm{HC}$ & 15 & 70 & 6.94 & 40 & - & - & - & - & & & & & 1.8 & 2.44 \\
\hline \multirow{2}{*}{ Timmer, 2018} & \multirow{2}{*}{$\begin{array}{l}\text { Stroop-like } \\
\text { incentive task } \\
\text { switching }\end{array}$} & PD & 23 & 61 & 7.4 & 39.13 & 4.5 & 2.2 & 21.8 & 6.7 & MMSE & 28.5 & 1.3 & $\mathrm{BDI}$ & 4.1 & 2.3 \\
\hline & & $\mathrm{HC}$ & 23 & 60.9 & 5.9 & 39.13 & - & - & - & - & & 28.8 & 1.2 & & 3.1 & 2.1 \\
\hline \multicolumn{4}{|c|}{ Response vigor in Parkinson's with psychiatric syndrome vs without } & \multicolumn{2}{|c|}{ Age } & & \multicolumn{2}{|c|}{ Disease duration } & \multicolumn{2}{|c|}{ UPDRS } & \multicolumn{3}{|c|}{ Cognition } & \multicolumn{3}{|c|}{ Depression } \\
\hline Study & Task & Group & $\mathrm{n}$ & Mean & SD & $\begin{array}{c}\% \\
\text { female }\end{array}$ & Mean & SD & Mean & SD & $\begin{array}{l}\text { Cognitive } \\
\text { measure }\end{array}$ & Mean & SD & $\begin{array}{c}\text { Depression } \\
\text { measure }\end{array}$ & Mean & SD \\
\hline \multirow{2}{*}{ Drew, 2020} & \multirow{2}{*}{$\begin{array}{l}\text { Speed of gaze } \\
\text { shifting task }\end{array}$} & PD & 26 & 67.19 & 5.92 & 26.9 & 4.87 & 4.09 & 18.62 & 9.38 & MoCA & 27.77 & 1.95 & $\mathrm{BDI}$ & 13 & 7.1 \\
\hline & & $I C D$ & 23 & 63.7 & 7.56 & 47.82 & 8.71 & 4.25 & 24.22 & 16.94 & & 27.39 & 2.55 & & 12.26 & 5.84 \\
\hline \multirow{2}{*}{ Evans, 2010} & \multirow{2}{*}{$\begin{array}{c}\text { Card arranging } \\
\text { reward responsivity } \\
\text { test }\end{array}$} & $\mathrm{PD}$ & 20 & 59.5 & 7.9 & & 13.6 & 8 & 19.2 & 2.5 & MMSE & 29.1 & $(27-30)$ & GDS & 9 & 4.9 \\
\hline & & DDS & 20 & 55.4 & 7.6 & & 14 & 5.7 & 22.7 & 2.5 & & 29 & $(24-30)$ & & 17.6 & 6.4 \\
\hline \multirow{2}{*}{ Lawrence, 2011} & \multirow{2}{*}{ Spatial search task } & PD & 10 & 59.6 & 6.4 & & & . & 25.6 & 11 & MMPD & 25.6 & 11 & GDS & 4.7 & 2 \\
\hline & & Apathy & 10 & 61.7 & 6.1 & & & . & 28.2 & 11.4 & & 29.3 & 2.8 & & 8.2 & 3.5 \\
\hline \multirow{2}{*}{ Muhammed, 2016} & \multirow{2}{*}{$\begin{array}{l}\text { Speed of gaze } \\
\text { shifting task }\end{array}$} & $\mathrm{PD}$ & 16 & 65.9 & 5.6 & 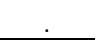 & 6.8 & 4.6 & 18.4 & 2.6 & MoCA & 28.2 & 1.6 & $\mathrm{BDI}$ & 11.7 & 5.5 \\
\hline & & Apathy & 14 & 67.3 & 6.4 & & 3.2 & 2.2 & 20.4 & 2.4 & & 27.8 & 2.3 & & 15.2 & 7.8 \\
\hline \multirow{2}{*}{ Timmer, 2018} & \multirow{2}{*}{$\begin{array}{l}\text { Stroop-like } \\
\text { incentive task } \\
\text { switching }\end{array}$} & $\mathrm{PD}$ & 23 & 61 & 7.4 & 39.13 & 4.5 & 2.2 & 21.8 & 6.7 & MMSE & 28.5 & 1.3 & $\mathrm{BDI}$ & 4.1 & 2.3 \\
\hline & & Depression & 22 & 58.4 & 5.7 & 36.36 & 5 & 3.5 & 23.1 & 9.6 & & 28.4 & 1.4 & & 9.6 & 6.1 \\
\hline
\end{tabular}

mental state examination, MoCA = Montreal cognitive assessment, MMPD $=$ Mini-mental Parkinson's examination BDI = Beck depression inventory, GDS $=$ Geriatric depression scale 
Supplement table 4. Study summary statistics, tasks and measures included in the Meta-Analysis of Parkinson's versus healthy controls.

\begin{tabular}{|c|c|c|c|c|c|c|c|c|c|c|c|c|c|c|}
\hline \multicolumn{13}{|c|}{ Reward processing summary statistics for Parkinson's disease patients versus healthy controls } & \multicolumn{2}{|c|}{ Tasks and Measures Included in the Meta-Analysis. } \\
\hline Author, Year & $\begin{array}{l}\text { Categ } \\
\text { ory }\end{array}$ & OFF/ON & $\begin{array}{l}\mathrm{N} \\
(\mathrm{HC}) \\
\end{array}$ & $\begin{array}{ll}N \\
(\mathrm{PD}) \\
\end{array}$ & $\mathrm{t}$ & $\mathrm{F}$ & $\begin{array}{l}\mathrm{M} \\
(\mathrm{HC}) \\
\end{array}$ & $\begin{array}{l}\mathrm{SD} \\
(\mathrm{HC})\end{array}$ & $\begin{array}{l}\mathrm{M} \\
(\mathrm{PD}) \\
\end{array}$ & \begin{tabular}{|l|}
$\mathrm{SD}$ \\
$(\mathrm{PD})$ \\
\end{tabular} & $d$ & Vard & Task & Measure \\
\hline Bayard et al, 2016 (ON) & ov & ON & 96 & 78 & & & 6.90 & 7.65 & 2.77 & 8.41 & 0.52 & 0.02 & Game of dice task & Net score \\
\hline Chong et al, 2015 (ON) & ov & ON & 26 & 26 & & & & & & & 0.35 & 0.08 & $\begin{array}{l}\text { Effort based decision making task } \\
\text { (apple gathering) }\end{array}$ & $\begin{array}{l}\text { Effort indifference point (mean effect size across } \\
\text { stake levels, fig 4) }\end{array}$ \\
\hline Chong et al, 2015 (OFF) & ov & OFF & 26 & 26 & & $\begin{array}{r}2.7 \\
0\end{array}$ & & & & & 0.46 & 0.08 & $\begin{array}{l}\text { Effort based decision making task } \\
\text { (apple gathering) }\end{array}$ & Effort indifference point \\
\hline Brandt et al, 2015 (ON) & ov & ON & 15 & 15 & & & $\begin{array}{r}14.9 \\
3 \\
\end{array}$ & $\begin{array}{r}15.7 \\
2\end{array}$ & $\begin{array}{r}- \\
8.67 \\
\end{array}$ & $\begin{array}{r}17.6 \\
7 \\
\end{array}$ & 1.41 & 0.17 & Game of dice task & Net score (fig 1) \\
\hline $\begin{array}{l}\text { Kobayashi et al, } 2019 \\
\text { (ON) }\end{array}$ & ov & ON & 21 & 15 & & & 0.21 & 0.78 & 0.30 & 0.66 & 0.12 & 0.11 & Economic choice task & $\begin{array}{l}\text { Relative risk aversion coefficient (fig 2C, first } \\
\text { session) }\end{array}$ \\
\hline $\begin{array}{l}\text { Kobayashi et al, } 2019 \\
\text { (OFF) }\end{array}$ & ov & OFF & 21 & 15 & & & 0.21 & 0.78 & 0.97 & 0.70 & 1.02 & 0.13 & Economic choice task & $\begin{array}{l}\text { Relative risk aversion coefficient (fig 2C, first } \\
\text { session) }\end{array}$ \\
\hline $\begin{array}{l}\text { Le Heron et al, } 2018 \\
\text { (ON) }\end{array}$ & ov & ON & 32 & 18 & $\begin{array}{r}1.3 \\
0\end{array}$ & & & & & & 0.38 & 0.09 & $\begin{array}{l}\text { Effort based decision making task } \\
\text { (apple gathering) }\end{array}$ & Mean difference in proportion of offers accepted \\
\hline $\begin{array}{l}\text { Mcguigan et al, } 2018 \\
\text { (ON) }\end{array}$ & ov & ON & 20 & 20 & $\begin{array}{r}- \\
0.3 \\
2\end{array}$ & & & & & & 0.10 & 0.10 & Cognitive effort task & Mean difference in k-value \\
\hline $\begin{array}{l}\text { Mcguigan et al, } 2018 \\
\text { (OFF) }\end{array}$ & ov & OFF & 20 & 20 & $\begin{array}{r}2.7 \\
9\end{array}$ & & & & & & 0.88 & 0.11 & Cognitive effort task & Mean difference in k-value \\
\hline Sharp et al, 2013 (ON) & ov & ON & 18 & 18 & $\begin{array}{l}0.4 \\
3\end{array}$ & & & & & & 0.14 & 0.07 & Vancouver gambling task & Gain phase adjust y intercept \\
\hline Sharp et al, 2013 (OFF) & ov & OFF & 18 & 18 & $\begin{array}{l}1.7 \\
9 \\
\end{array}$ & & & & & & 0.60 & 0.07 & Vancouver gambling task & Gain phase adjust y intercept \\
\hline Torta et al, 2009 (ON) & ov & ON & 13 & 15 & & & & & & & 0.24 & 0.14 & Cambridge gamble task & Average bet (across risk levels) \\
\hline Torta et al, 2009 (OFF) & ov & OFF & 13 & 15 & & & & & & & 0.23 & 0.14 & Cambridge gamble task & Average bet (across risk levels) \\
\hline Cools et al, 2003 (ON) & ov & ON & 12 & 12 & & & $\begin{array}{l}54.9 \\
1\end{array}$ & $\begin{array}{l}12.0 \\
9\end{array}$ & $\begin{array}{l}59.6 \\
5 \\
\end{array}$ & $\begin{array}{ll}16.1 \\
1\end{array}$ & 0.33 & 0.17 & Incentivised decision making task & $\begin{array}{l}\text { Mean \% bets across ascending \& descending } \\
\text { conditions }\end{array}$ \\
\hline Cools et al, 2003 (OFF) & ov & OFF & 12 & 12 & & & $\begin{array}{l}54.9 \\
1\end{array}$ & $\begin{array}{l}12.0 \\
9\end{array}$ & $\begin{array}{ll}58.9 \\
5\end{array}$ & $\begin{array}{l}15.7 \\
3\end{array}$ & 0.29 & 0.17 & Incentivised decision making task & $\begin{array}{l}\text { Mean \% bets across ascending \& descending } \\
\text { conditions }\end{array}$ \\
\hline Le Bouc et al, 2016 (ON) & ov & ON & 25 & 24 & & & $\begin{array}{r}80.8 \\
9 \\
\end{array}$ & 7.75 & $\begin{array}{r}78.5 \\
6 \\
\end{array}$ & $\begin{array}{r}12.4 \\
9 \\
\end{array}$ & 0.23 & 0.08 & $\begin{array}{l}\text { Effort based decision making } \\
\text { task: Binary choice task }\end{array}$ & $\begin{array}{l}\begin{array}{l}\text { Choice of effort level at the highest stake level. } \\
\text { (fig 4D) }\end{array} \\
\end{array}$ \\
\hline $\begin{array}{l}\text { Le Bouc et al, } 2016 \\
\text { (OFF) }\end{array}$ & ov & OFF & 25 & 24 & $\begin{array}{r}3.5 \\
1 \\
\end{array}$ & & & & & & 1.00 & 0.09 & $\begin{array}{l}\text { Effort based decision making } \\
\text { task: Binary choice task }\end{array}$ & $\begin{array}{l}\text { Choice of effort level at the highest stake level. } \\
\text { (fig 4D) }\end{array}$ \\
\hline $\begin{array}{l}\text { Castrioto et al, } 2015 \\
\text { (ON) }\end{array}$ & RL & ON & 24 & 20 & & & 4.38 & $\begin{array}{r}40.2 \\
2 \\
\end{array}$ & $\begin{array}{r}- \\
1.79 \\
\end{array}$ & $\begin{array}{r}44.7 \\
2 \\
\end{array}$ & 0.15 & 0.09 & lowa Gambling Task & Baseline final round mean score. (fig $1 \mathrm{~A})$ \\
\hline $\begin{array}{l}\text { lastrioto et al, } 2015 \\
\text { (OFF) }\end{array}$ & RL & OFF & 24 & 20 & & & 4.38 & $\begin{array}{r}40.2 \\
2 \\
\end{array}$ & 0.98 & $\begin{array}{r}40.3 \\
4 \\
\end{array}$ & 0.08 & 0.09 & lowa Gambling Task & Baseline final round mean score. (fig $1 \mathrm{~A})$ \\
\hline $\begin{array}{l}\text { Balusubramani et al, } \\
2015 \text { (ON) }\end{array}$ & $\mathrm{RL}$ & ON & 20 & 14 & & & $\begin{array}{r}64.1 \\
0\end{array}$ & $\begin{array}{r}84.8 \\
4\end{array}$ & $\begin{array}{r}61.5 \\
4\end{array}$ & $\begin{array}{r}78.6 \\
9\end{array}$ & 0.03 & 0.12 & $\begin{array}{l}\text { Probabilistic rewarded } \\
\text { categorisation learning task }\end{array}$ & \% Optimality during expected reward (fig 3A) \\
\hline $\begin{array}{l}\text { Balusubramani et al, } \\
2015 \text { (OFF) }\end{array}$ & RL & OFF & 20 & 26 & & & $\begin{array}{r}64.1 \\
0\end{array}$ & $\begin{array}{r}84.8 \\
4\end{array}$ & $\begin{array}{r}43.0 \\
7 \\
\end{array}$ & $\begin{array}{r}99.3 \\
8 \\
\end{array}$ & 0.23 & 0.09 & $\begin{array}{l}\text { Probabilistic rewarded } \\
\text { categorisation learning task }\end{array}$ & \% Optimality during expected reward (fig 3A) \\
\hline Bodi et al, 2009 (ON) & RL & Medicated & 20 & 22 & & & $\begin{array}{r}81.1 \\
8\end{array}$ & $\begin{array}{r}18.4 \\
3 \\
\end{array}$ & $\begin{array}{r}89.1 \\
8 \\
\end{array}$ & $\begin{array}{r}17.1 \\
2 \\
\end{array}$ & $\begin{array}{r}- \\
0.45 \\
\end{array}$ & 0.10 & $\begin{array}{l}\text { Feedback-based probabilistic } \\
\text { classification task }\end{array}$ & Final block \% optimal decisions (fig 2) \\
\hline $\begin{array}{l}\text { Bodi et al, } 2009 \text { (Never } \\
\text { medicated) }\end{array}$ & $\mathrm{RL}$ & $\begin{array}{l}\text { Never } \\
\text { medicated }\end{array}$ & 20 & 26 & & & $\begin{array}{r}81.1 \\
8 \\
\end{array}$ & $\begin{array}{r}18.4 \\
3 \\
\end{array}$ & $\begin{array}{r}59.5 \\
3 \\
\end{array}$ & $\begin{array}{r}18.6 \\
1 \\
\end{array}$ & 1.17 & 0.10 & $\begin{array}{l}\text { Feedback-based probabilistic } \\
\text { classification task }\end{array}$ & Final block \% optimal decisions (fig 2) \\
\hline Buelow et al, 2014 (ON) & RL & ON & 14 & 24 & & & & & & & 1.38 & 0.14 & lowa Gambling Task & Final block score (authors provided) \\
\hline
\end{tabular}




\begin{tabular}{|c|c|c|c|c|c|c|c|c|c|c|c|c|c|c|}
\hline Piray et al, 2014 (ON) & $\mathrm{RL}$ & ON & 20 & 15 & & & 0.12 & 0.16 & 0.14 & 0.20 & $0.10^{-}$ & 0.12 & Probabilistic learning task & Actor's learning rate (fig 6C) \\
\hline Piray et al, 2014 (OFF) & $\mathrm{RL}$ & OFF & 20 & 25 & & & 0.12 & 0.16 & 0.08 & 0.13 & 0.29 & 0.09 & Probabilistic learning task & Actor's learning rate (fig 6C) \\
\hline Yildirim et al, 2020 (ON) & $\mathrm{RL}$ & ON & 37 & 39 & & & 0.65 & 5.72 & 0.11 & 5.77 & 0.13 & 0.05 & lowa Gambling Task & Final block mean score (author provided) \\
\hline $\begin{array}{l}\text { Gescheidt et al, } 2012 \\
\text { (ON) }\end{array}$ & RL & ON & 20 & 19 & & & $\begin{array}{r}10.3 \\
0\end{array}$ & $\begin{array}{r}29.4 \\
2\end{array}$ & 6.00 & $\begin{array}{r}25.2 \\
6 \\
\end{array}$ & 0.59 & 0.11 & lowa Gambling Task & Mean total score \\
\hline $\begin{array}{l}\text { Herzallah et al, } 2017 \\
\text { (ON) }\end{array}$ & $\mathrm{RL}$ & ON & 15 & 17 & & & $\begin{array}{r}76.4 \\
9\end{array}$ & $\begin{array}{r}17.3 \\
6\end{array}$ & $\begin{array}{r}72.1 \\
0\end{array}$ & $\begin{array}{r}16.6 \\
2\end{array}$ & 0.26 & 0.13 & $\begin{array}{l}\text { Feedback-based probabilistic } \\
\text { classification task }\end{array}$ & $\begin{array}{l}\text { Mean \% optimal responses for positive feedback } \\
\text { (fig 5A) }\end{array}$ \\
\hline $\begin{array}{l}\text { lousden et al, } 2010 \\
\text { (ON) }\end{array}$ & $\mathrm{RL}$ & ON & 20 & 18 & & & $\begin{array}{r}56.5 \\
0 \\
\end{array}$ & $\begin{array}{r}22.4 \\
0 \\
\end{array}$ & $\begin{array}{r}27.0 \\
0 \\
\end{array}$ & $\begin{array}{r}19.1 \\
0 \\
\end{array}$ & 1.41 & 0.13 & Salience Attribution Test & $\begin{array}{l}\text { Visual analogue scale rating for high probability } \\
\text { stimuli }\end{array}$ \\
\hline Mapelli et al, 2014 (ON) & RL & ON & 15 & 15 & & & 9.81 & $\begin{array}{r}10.1 \\
9\end{array}$ & 4.74 & 5.11 & 0.63 & 0.14 & lowa Gambling Task & Final block mean score (fig 1 ) \\
\hline Mimura et al, 2006 (ON) & $\mathrm{RL}$ & ON & 20 & 18 & & & 3.70 & 7.35 & 0.33 & 6.59 & 0.58 & 0.11 & lowa Gambling Task & Final 50 cards mean score \\
\hline $\begin{array}{l}\text { Pagonabarraga et al, } \\
2007 \text { (ON) }\end{array}$ & $\mathrm{RL}$ & ON & 31 & 35 & & & 6.10 & $\begin{array}{r}17.0 \\
0\end{array}$ & $\begin{array}{r}10 . \overline{8} \\
0\end{array}$ & $\begin{array}{r}19.0 \\
0\end{array}$ & 0.93 & 0.07 & lowa Gambling Task & Mean total score \\
\hline $\begin{array}{l}\text { Paz-Alonso et al, } 2019 \\
\text { (ON) }\end{array}$ & RL & ON & 18 & 17 & & & $\begin{array}{r}40.6 \\
0\end{array}$ & $\begin{array}{r}18.8 \\
2 \\
\end{array}$ & $\begin{array}{r}41.8 \\
0\end{array}$ & $\begin{array}{r}15.3 \\
8 \\
\end{array}$ & 0.07 & 0.11 & lowa Gambling Task & $\begin{array}{l}\text { Mean difference \% optimal choice, final block. } \\
\text { (supplement fig 1) }\end{array}$ \\
\hline $\begin{array}{l}\text { Garofalo et al, } 2017 \\
\text { (ON) }\end{array}$ & $\mathrm{RL}$ & ON & 24 & 17 & & & $\begin{array}{r}21.5 \\
0\end{array}$ & 5.26 & $\begin{array}{r}15.1 \\
5\end{array}$ & 6.68 & 1.08 & 0.11 & Instrumental conditioning task & Reward learning index (fig 2B) \\
\hline $\begin{array}{l}\text { Czernecki et al, } 2002 \\
\text { (ON) }\end{array}$ & RL & ON & 28 & 23 & & & 8.32 & $\begin{array}{r}11.0 \\
6\end{array}$ & 2.86 & $\begin{array}{r}16.5 \\
9 \\
\end{array}$ & 0.40 & 0.08 & lowa Gambling Task & Final block mean score (fig 1 , second session) \\
\hline $\begin{array}{l}\text { Czernecki et al, } 2002 \\
\text { (OFF) }\end{array}$ & RL & OFF & 28 & 23 & & & 8.32 & $\begin{array}{r}11.0 \\
6 \\
\end{array}$ & 5.36 & $\begin{array}{r}10.8 \\
9 \\
\end{array}$ & 0.27 & 0.08 & Iowa Gambling Task & Final block mean score (fig 1 , second session) \\
\hline Graef et al, 2010 (ON) & RL & ON & 15 & 14 & $\begin{array}{r}1.8 \\
0\end{array}$ & & $\begin{array}{r}68.4 \\
9\end{array}$ & $\begin{array}{r}14.0 \\
7\end{array}$ & $\begin{array}{r}60.0 \\
7 \\
\end{array}$ & $\begin{array}{r}11.0 \\
8 \\
\end{array}$ & 0.67 & 0.15 & Instrumental learning task & $\begin{array}{l}\text { \% correct choices with constant reward } \\
\text { contingencies }\end{array}$ \\
\hline Graef et al, 2010 (OFF) & RL & OFF & 15 & 14 & $\begin{array}{r}2.1 \\
9 \\
\end{array}$ & & $\begin{array}{r}68.4 \\
9 \\
\end{array}$ & $\begin{array}{r}14.0 \\
7 \\
\end{array}$ & $\begin{array}{r}59.0 \\
5 \\
\end{array}$ & 8.86 & 0.81 & 0.15 & Instrumental learning task & $\begin{array}{l}\text { \% correct choices with constant reward } \\
\text { contingencies }\end{array}$ \\
\hline Thiel et al, 2003 (OFF) & $\mathrm{RL}$ & OFF & 5 & 5 & & & 53.6 & 15.8 & 61.2 & 17.1 & 0.46 & 0.41 & lowa Gambling Task & Mean number of advantageous cards selected. \\
\hline $\begin{array}{l}\text { Perretta et al, } 2005 \\
\text { (late PD) }\end{array}$ & RL & ON late PD & 19 & 16 & & & 7.80 & 1.31 & 6.80 & 1.60 & 0.69 & 0.12 & lowa Gambling Task & Mean total score \\
\hline $\begin{array}{l}\text { Perretta et al, } 2005 \\
\text { (early PD) }\end{array}$ & $\mathrm{RL}$ & ON early PD & 19 & 16 & & & 7.80 & 1.31 & 7.60 & 1.60 & 0.14 & 0.12 & lowa Gambling Task & Mean total score \\
\hline $\begin{array}{l}\text { Kobayakawa et al, } 2008 \\
\text { (ON) }\end{array}$ & $\mathrm{RL}$ & ON & 22 & 34 & & $\begin{array}{r}4.8 \\
0\end{array}$ & 4.90 & $\begin{array}{r}12.2 \\
0\end{array}$ & $\begin{array}{r}16.0 \\
0\end{array}$ & $\begin{array}{r}21.5 \\
7\end{array}$ & 0.60 & 0.08 & lowa Gambling Task & $\begin{array}{l}\text { Group difference in choice patterns } \\
\text { (advantageous - disadvantageous) }\end{array}$ \\
\hline $\begin{array}{l}\text { Kobayakawa et al, } 2010 \\
\text { (ON) }\end{array}$ & RL & ON & 22 & 14 & & $\begin{array}{r}2.9 \\
6 \\
\end{array}$ & & & & & 0.59 & 0.12 & lowa Gambling Task & $\begin{array}{l}\text { Group difference in choice patterns } \\
\text { (advantageous - disadvantageous) }\end{array}$ \\
\hline Poletti et al, 2010 (OFF) & $\mathrm{RL}$ & $\begin{array}{l}\text { OFF (de } \\
\text { novo) }\end{array}$ & 25 & 30 & & & 6.00 & 6.82 & 3.07 & $\begin{array}{r}12.0 \\
7\end{array}$ & 0.29 & 0.07 & lowa Gambling Task & Mean total score \\
\hline Delazer et al, 2009 (ON) & $\mathrm{RL}$ & ON & 20 & 20 & & $\begin{array}{r}8.8 \\
1\end{array}$ & & & & & 0.94 & 0.11 & lowa Gambling Task & $\begin{array}{l}\text { Group difference in choice patterns } \\
\text { (advantageous - disadvantageous) }\end{array}$ \\
\hline $\begin{array}{l}\text { Euteneuer et al, } 2009 \\
\text { (ON) }\end{array}$ & RL & ON & 23 & 21 & & $\begin{array}{r}0.6 \\
5 \\
\end{array}$ & & & & & 0.24 & 0.09 & lowa Gambling Task & $\begin{array}{l}\text { Group difference in choice patterns } \\
\text { (advantageous - disadvantageous) }\end{array}$ \\
\hline $\begin{array}{l}\text { Ibarretxe-Bilbao et al, } \\
2009(\mathrm{ON})\end{array}$ & $\mathrm{RL}$ & ON & 24 & 24 & & $\begin{array}{r}14 . \\
56 \\
\end{array}$ & & & & & 1.10 & 0.10 & Iowa Gambling Task & $\begin{array}{l}\text { Group difference in choice patterns } \\
\text { (advantageous - disadvantageous) }\end{array}$ \\
\hline Pignatti et al, 2012 (ON) & RL & ON & 16 & 15 & & & $\begin{array}{r}12.5 \\
0 \\
\end{array}$ & $\begin{array}{r}14.8 \\
7 \\
\end{array}$ & 7.47 & $\begin{array}{r}16.0 \\
6 \\
\end{array}$ & 0.33 & 0.13 & lowa Gambling Task & Total score over the last 50 choices \\
\hline Evens et al, 2015 (ON) & $\mathrm{RL}$ & ON & 32 & 32 & & & 7.31 & $\begin{array}{r}20.7 \\
8\end{array}$ & 2.09 & $\begin{array}{r}16.2 \\
7\end{array}$ & 0.28 & 0.06 & lowa Gambling Task & Mean total score \\
\hline
\end{tabular}




\begin{tabular}{|c|c|c|c|c|c|c|c|c|c|c|c|c|c|c|}
\hline Xi et al, 2015 (ON) & RL & ON & 15 & 15 & & \begin{tabular}{r|}
10. \\
67 \\
\end{tabular} & & & & & 1.19 & 0.16 & lowa Gambling Task & $\begin{array}{l}\text { Group difference in choice patterns } \\
\text { (advantageous - disadvantageous) }\end{array}$ \\
\hline $\begin{array}{l}\text { Cavanagh et al, } 2017 \\
\text { (ON) }\end{array}$ & RL & ON & 28 & 28 & $\begin{array}{r}1.6 \\
4 \\
\end{array}$ & & & & & & 0.44 & 0.07 & Cost of conflict task & $\begin{array}{l}\% \text { selection of most (A) vs least }(D) \text { rewarding } \\
\text { stimuli }\end{array}$ \\
\hline $\begin{array}{l}\text { Cavanagh et al, } 2017 \\
\text { (OFF) }\end{array}$ & RL & OFF & 28 & 28 & $\begin{array}{r}1.4 \\
4\end{array}$ & & & & & & 0.38 & 0.07 & Cost of conflict task & $\begin{array}{l}\text { \% selection of most (A) vs least (D) rewarding } \\
\text { stimuli }\end{array}$ \\
\hline $\begin{array}{l}\text { Muhammed et al, } 2016 \\
\text { (ON) }\end{array}$ & RV & ON & 31 & 30 & $\begin{array}{r}5.5 \\
0 \\
\end{array}$ & & & & & & 0.60 & 0.07 & $\begin{array}{l}\text { Rewarded speed of gaze shifting } \\
\text { task }\end{array}$ & Reward sensitivity in peak velocity \\
\hline Timmer et al, 2018 (ON) & RV & ON & 23 & 23 & & & 5.00 & 4.50 & $\begin{array}{l}14.4 \\
0\end{array}$ & 4.40 & 2.11 & 0.14 & $\begin{array}{l}\text { Rewarded task switching } \\
\text { paradigm }\end{array}$ & Reward related speeding 'repeat' condition \\
\hline $\begin{array}{l}\text { Timmer et al, } 2018 \\
\text { (OFF) }\end{array}$ & RV & OFF & 23 & 23 & & & 5.00 & 4.50 & 3.80 & 4.70 & 0.26 & 0.09 & $\begin{array}{l}\text { Rewarded task switching } \\
\text { paradigm }\end{array}$ & Reward related speeding 'repeat' condition \\
\hline
\end{tabular}

Supplement table 5. Study summary statistics, tasks and measures included in the Meta-Analysis of Parkinson's with \& without psychiatric syndrome

\begin{tabular}{|c|c|c|c|c|c|c|c|c|c|c|c|c|c|c|}
\hline \multicolumn{13}{|c|}{ Reward processing summary statistics for Parkinson's disease versus Parkinson's disease plus psychiatric syndrome } & \multicolumn{2}{|c|}{ Tasks and Measures Included in the Meta-Analysis. } \\
\hline Author, Year & Category & $\begin{array}{l}\text { Psychiatric } \\
\text { syndrome }\end{array}$ & $N(P D)$ & $\begin{array}{l}\mathrm{N} \\
(\mathrm{PD}+\mathrm{PSY} \\
\mathrm{CH})\end{array}$ & $\mathrm{t}$ & $\mathrm{F}$ & $\begin{array}{l}M \\
(P D)\end{array}$ & $\begin{array}{l}\text { SD } \\
(P D)\end{array}$ & $\begin{array}{l}M(P D \\
+ \text { Psych) }\end{array}$ & $\begin{array}{l}\text { SD } \\
\text { (PD+Psyc } \\
\text { h) }\end{array}$ & d & Vard & Task & Measure \\
\hline $\begin{array}{l}\text { Balusubramani } \\
\text { et al, } 2015 \text { (ON) }\end{array}$ & RL & ICD & 14 & 16 & & & $\begin{array}{r}61.5 \\
4 \\
\end{array}$ & $\begin{array}{r}78.6 \\
9\end{array}$ & 78.97 & 61.56 & -0.25 & 0.13 & $\begin{array}{l}\text { Probabilistic reward and } \\
\text { punishment learning task }\end{array}$ & $\begin{array}{l}\text { \% Optimality during expected reward (fig } \\
3 A \text { ) }\end{array}$ \\
\hline $\begin{array}{l}\text { Piray et al, } \\
2014 \text { (ON) } \\
\end{array}$ & RL & ICD & 15 & 16 & & & $\begin{array}{r}0.13 \\
5 \\
\end{array}$ & $\begin{array}{r}0.19 \\
8 \\
\end{array}$ & 0.067 & 0.06 & 0.47 & 0.13 & Probabilistic learning task & Actor's learning rate (fig 6C) \\
\hline $\begin{array}{l}\text { Housden et al, } \\
2010 \text { (ON) }\end{array}$ & $\mathrm{RL}$ & ICD & 18 & 18 & & & 27 & 19.1 & 42.7 & 19.7 & -0.81 & 0.12 & Salience Attribution Test & $\begin{array}{l}\text { Visual analogue scale rating for high } \\
\text { probability stimuli }\end{array}$ \\
\hline $\begin{array}{l}\text { Biars et al, } 2019 \\
\text { (ON) }\end{array}$ & RL & ICD & 24 & 24 & & & 3.5 & 9.7 & 2.75 & 8.2 & 0.08 & 0.08 & lowa Gambling Task & Final block score (authors provided) \\
\hline $\begin{array}{l}\text { Balconi et al, } \\
2018 \text { (ON) }\end{array}$ & $\mathrm{RL}$ & PG & 20 & 17 & & $\begin{array}{r}7 . \\
60 \\
\end{array}$ & & & & & 0.91 & 0.12 & lowa Gambling Task & $\begin{array}{l}\text { Group difference in choice patterns } \\
\text { (advantageous - disadvantageous) }\end{array}$ \\
\hline $\begin{array}{l}\text { Paz-Alonso et } \\
\text { al, } 2019 \text { (ON) }\end{array}$ & RL & ICD & 17 & 18 & & & $\begin{array}{r}41.8 \\
0\end{array}$ & $\begin{array}{r}15.3 \\
8\end{array}$ & 27.00 & 13.94 & 1.01 & 0.13 & lowa Gambling Task & $\begin{array}{l}\text { Mean difference \% optimal choice, final } \\
\text { block. (supplement fig 1) }\end{array}$ \\
\hline $\begin{array}{l}\text { Pineau et al, } \\
2016 \text { (ON) }\end{array}$ & $\mathrm{RL}$ & ICD & 20 & 17 & & & $\begin{array}{r}15.0 \\
0\end{array}$ & $\begin{array}{r}15.5 \\
6\end{array}$ & 14.00 & 9.63 & 0.08 & 0.11 & lowa Gambling Task & $\begin{array}{l}\text { Mean number of cards selected from } \\
\text { winning deck }\end{array}$ \\
\hline $\begin{array}{l}\text { Bentivoglio et } \\
\text { al, } 2012 \text { (ON) }\end{array}$ & $\mathrm{RL}$ & ICD & 17 & 17 & & & 8.40 & $\begin{array}{r}22.1 \\
0 \\
\end{array}$ & -4.60 & 33.10 & 0.46 & 0.12 & Iowa Gambling Task & Mean total score \\
\hline $\begin{array}{l}\text { Rossi et al, } 2010 \\
\text { (ON) }\end{array}$ & $\mathrm{RL}$ & PG & 13 & 7 & $\begin{array}{r}2 . \\
60 \\
\end{array}$ & & & & & & 1.22 & 0.26 & Iowa Gambling Task & Final block mean net score \\
\hline $\begin{array}{l}\text { Garofalo et al, } \\
2017 \text { (ON) }\end{array}$ & $\mathrm{RL}$ & Psychosis & 17 & 12 & & & $\begin{array}{r}15.1 \\
5 \\
\end{array}$ & 6.68 & 8.67 & 8.34 & 0.88 & 0.16 & Instrumental conditioning task & Reward learning index (fig 2B) \\
\hline $\begin{array}{l}\text { Sáez-Francàs et } \\
\text { al, } 2014 \text { (ON) }\end{array}$ & RL & Fatigue & 56 & 33 & & & 1.79 & $\begin{array}{r}14.8 \\
0 \\
\end{array}$ & -4.18 & 11.13 & 0.44 & 0.05 & Iowa Gambling Task & Final three blocks mean net score \\
\hline $\begin{array}{l}\text { Martinez-Horta } \\
\text { et al, } 2013 \text { (ON) }\end{array}$ & $\mathrm{RL}$ & Apathy & 17 & 20 & & & 1.82 & 5.80 & 4.10 & 4.80 & -1.12 & 0.13 & lowa Gambling Task & Final block score \\
\hline $\begin{array}{l}\text { Buelow et al, } \\
2014 \text { (ON) }\end{array}$ & $\mathrm{RL}$ & Apathy & 14 & 10 & & & $\begin{array}{r}22.1 \\
2 \\
\end{array}$ & $\begin{array}{r}16.8 \\
0 \\
\end{array}$ & -24.00 & 14.87 & 2.88 & 0.34 & lowa Gambling Task & Final block score (authors provided) \\
\hline $\begin{array}{l}\text { Herzallah et al, } \\
2017 \text { (ON) }\end{array}$ & $\mathrm{RL}$ & MDD & 17 & 13 & & & $\begin{array}{r}72.1 \\
0\end{array}$ & $\begin{array}{r}16.6 \\
2\end{array}$ & 49.30 & 22.14 & 1.19 & 0.16 & $\begin{array}{l}\text { Feedback-based probabilistic } \\
\text { classification task }\end{array}$ & $\begin{array}{l}\text { Mean \% optimal responses for positive } \\
\text { feedback (fig } 5 \mathrm{~A} \text { ) }\end{array}$ \\
\hline $\begin{array}{l}\text { Timmer et al, } \\
2017 \text { (ON) }\end{array}$ & $\mathrm{RL}$ & MDD & 22 & 19 & & & 0.10 & 0.06 & 0.07 & 0.06 & -0.50 & 0.10 & $\begin{array}{l}\text { Deterministic reversal learning } \\
\text { paradigm }\end{array}$ & Error rate for expected reward \\
\hline
\end{tabular}




\begin{tabular}{|c|c|c|c|c|c|c|c|c|c|c|c|c|c|c|}
\hline $\begin{array}{l}\text { Poletti et al, } \\
2011 \text { (OFF) }\end{array}$ & RL & Alexithymia & 12 & 12 & & & 4.17 & 8.37 & 3.00 & 6.35 & 0.16 & 0.17 & lowa Gambling Task & Final block score \\
\hline $\begin{array}{l}\text { Kobayashi et al, } \\
2019 \text { (ON) }\end{array}$ & ov & ICD & 15 & 10 & & & 0.30 & 0.66 & -0.19 & 0.17 & -0.93 & 0.18 & Economic choice task & $\begin{array}{l}\text { Relative risk aversion coefficient (fig } 2 \mathrm{C} \text {, } \\
\text { first session) }\end{array}$ \\
\hline $\begin{array}{l}\text { Le Heron et al, } \\
2018 \text { (ON) }\end{array}$ & ov & Apathy & 18 & 21 & $\begin{array}{r}2 . \\
33\end{array}$ & & & & & & 0.75 & 0.11 & $\begin{array}{l}\text { Effort based decision making } \\
\text { task (apple gathering) }\end{array}$ & $\begin{array}{l}\text { Mean difference in proportion of offers } \\
\text { accepted }\end{array}$ \\
\hline $\begin{array}{l}\text { Voon et al, } \\
2011 \text { (ON) }\end{array}$ & ov & ICD & 14 & 14 & & & & & & & -0.68 & 0.14 & Gambling task & $\begin{array}{l}\text { Proportion of risky choices in gain phase } \\
\text { (across risk levels, fig 2A) }\end{array}$ \\
\hline $\begin{array}{l}\text { Timmer et al, } \\
2018 \text { (ON) }\end{array}$ & RV & MDD & 23 & 22 & & & $\begin{array}{r}14.4 \\
0\end{array}$ & 4.40 & 17.70 & 3.90 & -0.79 & 0.10 & $\begin{array}{l}\text { Rewarded task-switching } \\
\text { paradigm }\end{array}$ & Reaction time reward benefit (repeat) \\
\hline $\begin{array}{l}\text { Evans et al, } \\
2010 \text { (ON) }\end{array}$ & RV & DDS & 20 & 20 & & $\begin{array}{r}12 \\
.7\end{array}$ & & & & & -1.13 & 0.12 & $\begin{array}{l}\text { Card arranging reward } \\
\text { responsivity objective test }\end{array}$ & Reward responsivity \\
\hline $\begin{array}{l}\text { Drew et al, } \\
2020 \text { (ON) }\end{array}$ & RV & ICD & 26 & 23 & & $\begin{array}{r}2 . \\
78\end{array}$ & & & & & -0.48 & 0.08 & $\begin{array}{l}\text { Rewarded speed of gaze } \\
\text { shifting task }\end{array}$ & Residual velocity reward sensitivity \\
\hline $\begin{array}{l}\text { Lawrence et al, } \\
2011 \text { (ON) }\end{array}$ & RV & Apathy & 10 & 10 & & $\begin{array}{c}0 . \\
38\end{array}$ & & & & & 0.20 & 0.28 & Rewarded spatial search task & Reward related speeding \\
\hline
\end{tabular}

\section{Supplement table 6. Study summary statistics, tasks and measures included in the Meta-Analysis of Parkinson's on and off dopaminergic} medication

\begin{tabular}{|c|c|c|c|c|c|c|c|c|}
\hline \multicolumn{7}{|c|}{$\begin{array}{l}\text { Reward processing summary statistics for Parkinson's disease patients ON vs OFF } \\
\text { dopaminergic medication }\end{array}$} & \multicolumn{2}{|c|}{ Tasks and Measures Included in the Meta-Analysis. } \\
\hline Author, Year & Category & $\mathrm{N}$ & $\mathrm{t}$ & $\mathrm{F}$ & d & Vard & Task & Measure \\
\hline Czernecki et al, 2002 & $\mathrm{RL}$ & 22 & & 0.05 & 0.048 & 0.046 & lowa Gambling Task & Number of advantageous minus disadvantageous choices \\
\hline Graef et al, 2010 & RL & 14 & -0.374 & & -0.100 & 0.072 & Instrumental learning task & $\%$ correct choices with constant reward contingencies \\
\hline Cavanagh et al, 2017 & RL & 28 & & 2.87 & 0.320 & 0.0375 & Value of volition task & $\begin{array}{l}\text { \% selection of A ( } 90 \% \text { reward) vs C ( } 70 \% \text { reward) - B ( } 10 \% \text { reward) } \\
\text { vs D ( } 30 \% \text { reward) stimuli }\end{array}$ \\
\hline Bodi et al, 2009 & RL & 26 & & 13.47 & 0.720 & 0.0385 & Feedback-based probabilistic classification task & Pattern of optimal decision selection over time \\
\hline Chong et al, 2015 & ov & 26 & & \multirow{3}{*}{$\begin{array}{l}25.9 \\
7.48\end{array}$} & 0.998 & 0.058 & $\begin{array}{l}\text { Effort based decision making task (apple } \\
\text { gathering) }\end{array}$ & Effort indifference point \\
\hline Kobayashi et al, 2019 & ov & 24 & & & 0.558 & 0.048 & Economic choice task & Relative risk aversion coefficient \\
\hline Le Heron et al, 2018 & ov & 39 & 2.45 & & 0.392 & 0.028 & $\begin{array}{l}\text { Effort based decision making task (apple } \\
\text { gathering) }\end{array}$ & Mean difference in proportion of offers accepted \\
\hline Mcguigan et al, 2018 & ov & 20 & 3.05 & & 0.682 & 0.062 & Cogntiive effort task & Mean difference in k-value \\
\hline Cools et al, 2003 & ov & 12 & & 5.9 & 0.701 & 0.104 & Incentivised decision making task & $\begin{array}{l}\text { Difference score of bets places in ascending and descending } \\
\text { conditions. }\end{array}$ \\
\hline Le Bouc et al, 2016 & ov & 20 & 2.76 & & 0.617 & 0.060 & $\begin{array}{l}\text { Effort based decision making task: Binary } \\
\text { choice task }\end{array}$ & Choice of effort level at the highest stake level. \\
\hline Sharp et al, 2013 & ov & 18 & 1.17 & & 0.276 & 0.058 & Vancouver gambling task & Adjusted y intercept \\
\hline Timmer et al, 2018 & RV & 23 & & 3.031 & 0.363 & 0.046 & Rewarded task switching paradigm & Reward related speeding 'repeat' condition \\
\hline Muhammed et al, 2016 & RV & 30 & & 10.8 & 0.600 & 0.039 & Rewarded speed of gaze shifting task & Reward sensitivity in peak velocity \\
\hline Evans et al, 2010 & RV & 38 & & 0.4 & 0.103 & 0.026 & $\begin{array}{l}\text { Card arranging reward responsivity objective } \\
\text { test }\end{array}$ & Reward responsivity \\
\hline Drew, 2020 & RV & 26 & & 5.178 & 0.446 & 0.042 & Rewarded speed of gaze shifting task & Residual velocity reward sensitivity \\
\hline
\end{tabular}




\section{Modified version of the Newcastle-Ottawa Scale for Assessing the Quality of Nonrandomized Studies in Meta-Analyses, used to assess} potential sources of bias.

Potential sources of bias were assessed using a modified version of the Newcastle-Ottawa Scale for Assessing the Quality of Nonrandomized Studies in Meta-Analyses. The studies are scored on:
1. PD Definition: Is the case definition adequate?
A) Cases were defined as PD according to a validated assessment tool/criteria or by an experienced clinician
B) Cases were defined as PD according to a validated assessment tool/criteria but the method for assessing PD status was not stated.
C) Cases were described as 'clinically' but no further description was given.

\section{PD Generality: Was a General sample of cases tested? \\ A) A General sample of PD was tested. \\ B) Recruitment of PD cases was restricted to a specific sub-sample (specific age range, hospitalised only etc.)}

\section{HC Selection: Selection of Controls}

A) Controls were selected from the same population as cases

B) Controls were not selected from the same population as cases

C) No description

\section{HC Definition: Definition of Controls}

A) $\mathrm{HC}$ were defined clearly defined as having no current or past psychopathology

B) Controls were not clearly defined as having no current or past psychopathology.

\section{Comparability (Comparability of cases and controls on the basis of the design or analysis)}

1. Does the study control for Age: Yes/No/Unclear

2. Does the study control for Gender: Yes/No/Unclear

3. Does the study control for IQ: Yes/No/Unclear

4. Does the study control for Socioeconomic status: Yes/No/Unclear

5. Does the study control for PD severity: Yes/No/Unclear

6. Does the study control for medication status: Yes/No/Unclear

Key: A, B, C

$\mathrm{Y}=$ yes, $\mathrm{N}=$ no, $\mathrm{N} / \mathrm{A}=$ Not applicable (such as if no healthy control group in study) 


\section{Supplement table 7. Quality rating of included studies}

\begin{tabular}{|c|c|c|c|c|c|c|c|c|c|c|c|c|}
\hline \multicolumn{3}{|c|}{ Study quality ratings } & \multicolumn{10}{|c|}{ Modified version of the Newcastle-Ottawa Scale } \\
\hline Study & Year & Category & PD Definition & PD Generality & HC Selection & HC Definition & Age & Gender & IQ & Socioeconomic status & PD severity & PD medication \\
\hline Bayard $^{1}$ & 2016 & OV & A & A & B & A & Y & $\mathrm{Y}$ & $\mathrm{N}$ & $\mathrm{N}$ & Y & $\mathrm{Y}$ \\
\hline Brandt $^{2}$ & 2015 & OV & A & A & $\mathrm{C}$ & B & $\mathrm{Y}$ & $\mathrm{Y}$ & $\mathrm{Y}$ & $\mathrm{N}$ & $\mathrm{Y}$ & $\mathrm{Y}$ \\
\hline Chong $^{3}$ & 2015 & OV & B & A & A & A & $\mathrm{Y}$ & $\mathrm{Y}$ & $\mathrm{N}$ & $\mathrm{N}$ & $\mathrm{Y}$ & $\mathrm{Y}$ \\
\hline Cools $^{4}$ & 2003 & OV & A & A & C & B & $\mathrm{Y}$ & $\mathrm{N}$ & $\mathrm{Y}$ & $\mathrm{N}$ & $\mathrm{Y}$ & $\mathrm{Y}$ \\
\hline Haagensen $^{5}$ & 2020 & OV & $\mathrm{C}$ & A & A & $B$ & $\mathrm{Y}$ & $Y$ & $\mathrm{~N}$ & $\mathrm{~N}$ & $\mathrm{Y}$ & $\mathrm{Y}$ \\
\hline Kobayashi $^{6}$ & 2019 & OV & $A$ & A & C & $B$ & $\mathrm{Y}$ & $Y$ & $\mathrm{~N}$ & $\mathrm{~N}$ & $\mathrm{Y}$ & $Y$ \\
\hline Le Bouc ${ }^{7}$ & 2016 & OV & C & $B$ & A & B & $\mathrm{Y}$ & $Y$ & $\mathrm{~N}$ & $\mathrm{~N}$ & $\mathrm{Y}$ & $\mathrm{Y}$ \\
\hline Le Heron ${ }^{8}$ & 2018 & OV & A & A & A & A & $Y$ & $Y$ & $\mathrm{~N}$ & $\mathrm{~N}$ & $\mathrm{Y}$ & $Y$ \\
\hline Mcguigan $^{9}$ & 2018 & OV & A & A & A & A & $Y$ & $Y$ & $\mathrm{~N}$ & $\mathrm{~N}$ & $Y$ & $Y$ \\
\hline Torta $^{10}$ & 2009 & OV & A & B & c & B & $Y$ & $Y$ & $\mathrm{~N}$ & $\mathrm{~N}$ & $Y$ & $Y$ \\
\hline Sharp ${ }^{11}$ & 2013 & OV & A & A & C & B & $Y$ & $\mathrm{~N}$ & $\mathrm{~N}$ & $\mathrm{~N}$ & $\mathrm{Y}$ & $\mathrm{Y}$ \\
\hline Voon $^{12}$ & 2011 & OV & A & $\mathrm{A}$ & $n / a$ & $\mathrm{n} / \mathrm{a}$ & $\mathrm{Y}$ & $Y$ & $\mathrm{~N}$ & $\mathrm{~N}$ & $\mathrm{Y}$ & $Y$ \\
\hline Balconi ${ }^{13}$ & 2018 & $\mathrm{RL}$ & A & A & $\mathrm{n} / \mathrm{a}$ & $\mathrm{n} / \mathrm{a}$ & $Y$ & $\mathrm{~N}$ & $\mathrm{~N}$ & $\mathrm{~N}$ & $\mathrm{Y}$ & $Y$ \\
\hline Balusubramani $i^{14}$ & 2015 & $\mathrm{RL}$ & C & A & c & A & $\mathrm{N}$ & $\mathrm{N}$ & $\mathrm{N}$ & $\mathrm{N}$ & $Y$ & $Y$ \\
\hline Bentivoglio ${ }^{15}$ & 2012 & $\mathrm{RL}$ & A & $A$ & $n / a$ & $n / a$ & $\mathrm{Y}$ & $Y$ & $\mathrm{Y}$ & $\mathrm{N}$ & $\mathrm{Y}$ & $\mathrm{Y}$ \\
\hline Biars' & 2019 & $\mathrm{RL}$ & $\mathrm{C}$ & $B$ & $\mathrm{n} / \mathrm{a}$ & $\mathrm{n} / \mathrm{a}$ & $Y$ & $Y$ & $\mathrm{Y}$ & $\mathrm{N}$ & $\mathrm{N}$ & $Y$ \\
\hline Bodi ${ }^{17}$ & 2009 & $\mathrm{RL}$ & C & B & c & A & $Y$ & $\mathrm{~N}$ & $Y$ & $Y$ & $Y$ & $Y$ \\
\hline Buelow $^{18}$ & 2014 & $\mathrm{RL}$ & A & A & A & A & $Y$ & $Y$ & $Y$ & $\mathrm{~N}$ & $Y$ & $Y$ \\
\hline Castrioto $^{19}$ & 2015 & $\mathrm{RL}$ & $\mathrm{C}$ & $B$ & $\mathrm{C}$ & B & $Y$ & $Y$ & $\mathrm{~N}$ & $\mathrm{~N}$ & $\mathrm{Y}$ & $\mathrm{Y}$ \\
\hline Cavanagh $^{20}$ & 2017 & $\mathrm{RL}$ & C & A & c & B & $Y$ & $Y$ & $Y$ & $\mathrm{~N}$ & $Y$ & $Y$ \\
\hline Czernecki $^{21}$ & 2002 & $\mathrm{RL}$ & A & B & B & A & $Y$ & $Y$ & $\mathrm{~N}$ & $\mathrm{~N}$ & $Y$ & $Y$ \\
\hline Delazer $^{22}$ & 2009 & $\mathrm{RL}$ & A & A & $\mathrm{C}$ & $A$ & $Y$ & $Y$ & $\mathrm{~N}$ & $\mathrm{~N}$ & $Y$ & $\mathrm{Y}$ \\
\hline Euteneuer $^{23}$ & 2009 & $\mathrm{RL}$ & $\mathrm{C}$ & A & C & $B$ & $\mathrm{Y}$ & $\mathrm{Y}$ & $\mathrm{N}$ & $\mathrm{N}$ & $\mathrm{Y}$ & $\mathrm{Y}$ \\
\hline Evens $^{24}$ & 2015 & $\mathrm{RL}$ & A & A & A & A & $Y$ & $Y$ & $\mathrm{~N}$ & $\mathrm{~N}$ & $Y$ & $Y$ \\
\hline Garofalo $^{25}$ & 2017 & $\mathrm{RL}$ & A & A & C & B & $Y$ & $Y$ & $Y$ & $\mathrm{~N}$ & $Y$ & $Y$ \\
\hline Gescheidt ${ }^{26}$ & 2012 & $\mathrm{RL}$ & A & A & $\mathrm{c}$ & $A$ & $Y$ & $Y$ & $\mathrm{~N}$ & $\mathrm{~N}$ & $Y$ & $Y$ \\
\hline Graef $^{27}$ & 2010 & $\mathrm{RL}$ & A & B & B & A & $Y$ & $Y$ & $\mathrm{~N}$ & $\mathrm{~N}$ & $Y$ & $Y$ \\
\hline Herzallah ${ }^{28}$ & 2017 & RL \& RV & B & A & A & A & $Y$ & $\mathrm{~N}$ & $\mathrm{~N}$ & $\mathrm{~N}$ & $Y$ & $\mathrm{~N}$ \\
\hline Housden $^{29}$ & 2010 & $\mathrm{RL}$ & $\mathrm{C}$ & A & A & $A$ & $Y$ & $Y$ & $\mathrm{Y}$ & $\mathrm{N}$ & $Y$ & $\mathrm{Y}$ \\
\hline Ibarretxe-Bilbao ${ }^{30}$ & 2009 & $\mathrm{RL}$ & A & A & A & B & $Y$ & $Y$ & $\mathrm{~N}$ & $\mathrm{~N}$ & $Y$ & $\mathrm{Y}$ \\
\hline Kobayakawa ${ }^{31}$ & 2008 & RL & $\mathrm{C}$ & A & A & A & $\mathrm{Y}$ & $\mathrm{Y}$ & $\mathrm{N}$ & $\mathrm{N}$ & $\mathrm{Y}$ & $\mathrm{Y}$ \\
\hline Kobayakawa ${ }^{32}$ & 2010 & $\mathrm{RL}$ & $\mathrm{C}$ & A & A & $A$ & $Y$ & $Y$ & $\mathrm{~N}$ & $\mathrm{~N}$ & $Y$ & $\mathrm{Y}$ \\
\hline Mapelli33 & 2014 & $\mathrm{RL}$ & A & A & A & A & $Y$ & $\mathrm{Y}$ & $\mathrm{N}$ & $\mathrm{N}$ & $Y$ & $\mathrm{Y}$ \\
\hline Martinez-Horta ${ }^{34}$ & 2013 & $\mathrm{RL}$ & A & A & $n / a$ & $n / a$ & $Y$ & $\mathrm{~N}$ & $\mathrm{~N}$ & $\mathrm{~N}$ & $Y$ & $Y$ \\
\hline Mimura 35 & 2006 & $\mathrm{RL}$ & $\mathrm{C}$ & A & $B$ & A & $\mathrm{Y}$ & $\mathrm{Y}$ & $\mathrm{N}$ & $\mathrm{N}$ & $\mathrm{Y}$ & $\mathrm{Y}$ \\
\hline Pagonabarraga ${ }^{36}$ & 2007 & $\mathrm{RL}$ & A & A & A & A & $\mathrm{Y}$ & $Y$ & $\mathrm{~N}$ & $\mathrm{~N}$ & $\mathrm{Y}$ & $\mathrm{Y}$ \\
\hline Paz-Alonso ${ }^{37}$ & 2019 & $\mathrm{RL}$ & A & A & A & B & $\mathrm{Y}$ & $Y$ & $\mathrm{Y}$ & $\mathrm{N}$ & $\mathrm{Y}$ & $\mathrm{Y}$ \\
\hline Perretta $^{38}$ & 2005 & $\mathrm{RL}$ & A & A & A & $A$ & $Y$ & $Y$ & $\mathrm{~N}$ & $\mathrm{~N}$ & $Y$ & $\mathrm{Y}$ \\
\hline Pignatt $\mathrm{B}^{39}$ & 2012 & $\mathrm{RL}$ & $A$ & $B$ & $\mathrm{C}$ & $B$ & $\mathrm{~N}$ & $\mathrm{~N}$ & $\mathrm{~N}$ & $\mathrm{~N}$ & $\mathrm{~N}$ & $\mathrm{~N}$ \\
\hline Pineau $^{40}$ & 2016 & $\mathrm{RL}$ & A & A & $n / a$ & $n / a$ & $\mathrm{Y}$ & $\mathrm{Y}$ & $\mathrm{N}$ & $\mathrm{N}$ & $Y$ & $\mathrm{Y}$ \\
\hline Piray 41 & 2014 & $\mathrm{RL}$ & $\mathrm{C}$ & $B$ & C & A & $Y$ & $\mathrm{~N}$ & $\mathrm{Y}$ & $\mathrm{N}$ & $Y$ & $\mathrm{~N}$ \\
\hline Poletti ${ }^{42}$ & 2010 & $\mathrm{RL}$ & A & A & C & $B$ & $\mathrm{Y}$ & $\mathrm{Y}$ & $\mathrm{N}$ & $\mathrm{N}$ & $Y$ & $Y$ \\
\hline Poletti ${ }^{43}$ & 2011 & $\mathrm{RL}$ & A & $B$ & $n / a$ & $n / a$ & $Y$ & $Y$ & $\mathrm{~N}$ & $\mathrm{~N}$ & $\mathrm{~N}$ & $\mathrm{Y}$ \\
\hline Rossi $^{44}$ & 2010 & $\mathrm{RL}$ & A & A & $n / a$ & $n / a$ & $Y$ & $Y$ & $\mathrm{~N}$ & $\mathrm{~N}$ & $Y$ & $\mathrm{Y}$ \\
\hline Sáez-Francàs ${ }^{45}$ & 2014 & $\mathrm{RL}$ & $\mathrm{C}$ & $A$ & $n / a$ & $n / a$ & $Y$ & $Y$ & $\mathrm{~N}$ & $\mathrm{~N}$ & $\mathrm{Y}$ & $\mathrm{N}$ \\
\hline Thiel $^{46}$ & 2003 & $\mathrm{RL}$ & C & A & C & A & $Y$ & $\mathrm{~N}$ & $\mathrm{~N}$ & $\mathrm{~N}$ & $Y$ & $\mathrm{Y}$ \\
\hline Timmer $^{47}$ & 2017 & $\mathrm{RL}$ & A & A & A & $A$ & $Y$ & $Y$ & $Y$ & $\mathrm{~N}$ & $Y$ & $Y$ \\
\hline $\mathrm{Xi}^{48}$ & 2015 & $\mathrm{RL}$ & A & B & $\mathrm{C}$ & B & $Y$ & $Y$ & $\mathrm{~N}$ & $\mathrm{~N}$ & $Y$ & $Y$ \\
\hline
\end{tabular}




\begin{tabular}{|l|l|l|l|l|l|l|l|l|l|l|l|l|} 
Yildirim $^{49}$ & 2020 & RL & A & A & B & A & Y & Y & N & N & Y \\
\hline Drew $^{50}$ & 2020 & RV & A & B & C & A & Y & Y & N & N & Y \\
\hline Evans $^{51}$ & 2010 & RV & A & A & B & B & Y & Y & N & N & Y & Y \\
\hline Lawrence $^{52}$ & 2011 & RV & A & A & n/a & n/a & Y & N & N & N & Y \\
\hline Muhammed $^{53}$ & 2016 & RV & C & A & B & B & Y & Y & Y & N & Y \\
\hline Renfroe $^{54}$ & 2016 & RV & B & B & A & A & Y & Y & N & N & Y \\
\hline Timmer $^{55}$ & 2018 & RV & A & A & A & B & Y & N & Y & N & N \\
\hline
\end{tabular}

\section{References}

1. Bayard, S. et al. A multidimensional approach to impulsivity in Parkinson's disease: measurement and structural invariance of the UPPS Impulsive Behaviour Scale. Psychol. Med. 46, 2931-2941 (2016).

2. J., B. et al. Betting on DBS: Effects of subthalamic nucleus deep brain stimulation on risk taking and decision making in patients with Parkinson's disease. Neuropsychology 29, 622-631 (2015).

3. Chong, T. T. J. et al. Dopamine enhances willingness to exert effort for reward in Parkinson's disease. Cortex 69, 40-46 (2015).

4. Cools, R., Barker, R. A., Sahakian, B. J. \& Robbins, T. W. L-Dopa medication remediates cognitive inflexibility, but increases impulsivity in patients with Parkinson's disease. Neuropsychologia 41, 1431-1441 (2003).

5. B.N., H. et al. Linking brain activity during sequential gambling to impulse control in Parkinson's disease. NeuroImage. Clin. 27, 102330 (2020).

6. Kobayashi, S. et al. Dopaminergic influences on risk preferences of Parkinson's disease patients. Cogn. Affect. Behav. Neurosci. 19, 88-97 (2019).

7. Le Bouc, R. et al. Computational Dissection of Dopamine Motor and Motivational Functions in Humans. J. Neurosci. 36, 6623-6633 (2016).

8. Le Heron, C. et al. Distinct effects of apathy and dopamine on effort-based decision-making in Parkinson's disease. Brain 141, 1455-1469 (2018).

9. S., M. et al. Dopamine restores cognitive motivation in Parkinson's disease. Brain 142, 719-732 (2019).

10. Torta, D. M. E. et al. On the role of dopamine replacement therapy in decision-making, working memory, and reward in Parkinson's disease: does the therapy-dose matter?. Brain Cogn. 71, 84-91 (2009).

11. Sharp, M. E. et al. Decisions under risk in Parkinson's disease: preserved evaluation of probability and magnitude. Neuropsychologia 51, 2679-2689 (2013).

12. Voon, V. et al. Dopamine agonists and risk: Impulse control disorders in Parkinson's; Disease. Brain (2011). doi:10.1093/brain/awr080

13. M., B. et al. Gambling behavior in Parkinson's Disease: Impulsivity, reward mechanism and cortical brain oscillations. Psychiatry Res. 270, 974-980 (2018).

14. Balasubramani, P. P. et al. Identifying the basal ganglia network model markers for medication-induced impulsivity in Parkinson's disease patients. PLoS One 10, e0127542 (2015).

15. Bentivoglio, A. R., Baldonero, E., Ricciardi, L., De Nigris, F. \& Daniele, A. Neuropsychological features of patients with Parkinson's disease and impulse control disorders. Neurol. Sci. (2013). doi:10.1007/s10072-012-1224-5

16. J.W., B. et al. Iowa Gambling Task Performance in Parkinson Disease Patients with Impulse Control Disorders. Arch. Clin. Neuropsychol. 34, 310-318 (2019). 
17. Bodi, N. et al. Reward-learning and the novelty-seeking personality: a between- and within-subjects study of the effects of dopamine agonists on young Parkinson's patients. Brain 132, 2385-2395 (2009).

18. Buelow, M. T. et al. The contribution of apathy and increased learning trials to risky decision-making in Parkinson's disease. Arch. Clin. Neuropsychol. 29, 100-109 (2014).

19. A., C. et al. Iowa gambling task impairment in Parkinson's disease can be normalised by reduction of dopaminergic medication after subthalamic stimulation. J. Neurol. Neurosurg. Psychiatry 86, 186-190 (2015).

20. Cavanagh, J. F. et al. Cognitive states influence dopamine-driven aberrant learning in Parkinson's disease. Cortex (2017). doi:10.1016/j.cortex.2017.02.021

21. V., C. et al. Motivation, reward, and Parkinson's disease: Influence of dopatherapy. Neuropsychologia 40, $2257-2267$ (2002).

22. Delazer, M. et al. Decision making under risk and under ambiguity in Parkinson's disease. Neuropsychologia (2009). doi:10.1016/j.neuropsychologia.2009.02.034

23. Euteneuer, F. et al. Dissociation of decision-making under ambiguity and decision-making under risk in patients with Parkinson's disease: A neuropsychological and psychophysiological study. Neuropsychologia (2009). doi:10.1016/j.neuropsychologia.2009.06.014

24. Evens, R. et al. The impact of Parkinson's disease and subthalamic deep brain stimulation on reward processing. Neuropsychologia (2015). doi:10.1016/j.neuropsychologia.2015.05.005

25. S., G. et al. Cortical and striatal reward processing in Parkinson's disease psychosis. Front. Neurol. 8, 156 (2017).

26. Gescheidt, T. et al. Iowa Gambling Task in patients with early-onset Parkinson's disease: strategy analysis. Neurol. Sci. 33, 1329-1335 (2012).

27. Graef, S. et al. Differential influence of levodopa on reward-based learning in Parkinson's disease. Front. Hum. Neurosci. 4, 169 (2010).

28. Herzallah, M. M. et al. Depression Reduces Accuracy While Parkinsonism Slows Response Time for Processing Positive Feedback in Patients with Parkinson's Disease with Comorbid Major Depressive Disorder Tested on a Probabilistic Category-Learning Task. Front. psychiatry 8, 84 (2017).

29. Housden, C. R. et al. Intact reward learning but elevated delay discounting in Parkinson's disease patients with impulsive-compulsive spectrum behaviors. Neuropsychopharmacology 35, 2155-2164 (2010).

30. Ibarretxe-Bilbao, N. et al. Neuroanatomical correlates of impaired decision-making and facial emotion recognition in early Parkinson's disease. Eur. J. Neurosci. (2009). doi:10.1111/j.1460-9568.2009.06892.x

31. Kobayakawa, M., Koyama, S., Mimura, M. \& Kawamura, M. Decision making in Parkinson's disease: Analysis of behavioral and physiological patterns in the Iowa Gambling task. Mov. Disord. (2008). doi:10.1002/mds.21865

32. Kobayakawa, M., Tsuruya, N. \& Kawamura, M. Sensitivity to reward and punishment in Parkinson's disease: An analysis of behavioral patterns using a modified version of the Iowa gambling task. Park. Relat. Disord. (2010). doi:10.1016/j.parkreldis.2010.04.011

33. Mapelli, D., Di Rosa, E., Cavalletti, M., Schiff, S. \& Tamburin, S. Decision and dopaminergic system: an ERPs study of Iowa gambling task in Parkinson's disease. Front. Psychol. 5, 684 (2014).

34. Martínez-Horta, S., Pagonabarraga, J., Fernández De Bobadilla, R., García-Sanchez, C. \& Kulisevsky, J. Apathy in Parkinson's disease: More than just executive dysfunction. J. Int. Neuropsychol. Soc. (2013). doi:10.1017/S1355617713000131

35. Mimura, M. et al. Impaired decision-making in Parkinson's disease. Parkinsonism Relat. Disord. 12, 169-175 (2006).

36. Pagonabarraga, J. et al. Controlled study of decision-making and cognitive impairment in Parkinson's disease. Mov. Disord. 22, 1430-1435 (2007).

37. Paz-Alonso, P. M. et al. Functional inhibitory control dynamics in impulse control disorders in Parkinson's disease. Mov. Disord. 35, 316-325 (2020). 
38. Perretta, J. G., Pari, G. \& Beninger, R. J. Effects of Parkinson Disease on Two Putative Nondeclarative Learning Tasks. Cogn. Behav. Neurol. (2005). doi:10.1097/01.wnn.0000187939.81541.1d

39. Pignatti, R. et al. Selective IGT decision-making impairment in a patient with juvenile Parkinson's disease and pathological gambling: A role for dopaminergic therapy? Neurocase (2012). doi:10.1080/13554794.2011.633529

40. Pineau, F. et al. Executive functioning and risk-taking behavior in Parkinson's disease patients with impulse control disorders. J. Neural Transm. 123, 573-581 (2016).

41. D., H. et al. Impulse control disorders in Parkinson's disease are associated with dysfunction in stimulus valuation but not action valuation. $J$. Neurosci. 34, 7814-7824 (2014).

42. Poletti, M. et al. Decision making in de novo Parkinson's disease. Mov. Disord. (2010). doi:10.1002/mds.23098

43. Poletti, M. et al. Alexithymia may modulate decision making in patients with de novo Parkinson's disease. Funct. Neurol. 26, 127-131 (2011).

44. Rossi, M. et al. Decision-making in Parkinson's disease patients with and without pathological gambling. Eur. J. Neurol. 17, 97-102 (2010).

45. Saez-Francas, N. et al. Relationship between poor decision-making process and fatigue perception in Parkinson's disease patients. J. Neurol. Sci. 337, 167-172 (2014).

46. Thiel, A. et al. Activation of basal ganglia loops in idiopathic Parkinson's disease: A PET study. J. Neural Transm. (2003). doi:10.1007/s00702-003-0041-7

47. Timmer, M. H. M., Sescousse, G., Van Der Schaaf, M. E., Esselink, R. A. J. \& Cools, R. Reward learning deficits in Parkinson's disease depend on depression. Psychol. Med. (2017). doi:10.1017/S0033291717000769

48. Xi, C. et al. Theory of mind and decision-making processes are impaired in Parkinson's disease. Behav. Brain Res. (2015). doi:10.1016/j.bbr.2014.11.035

49. E., Y. \& S., A. Decision-making and impulse-control disorders in parkinson's disease: Influence of dopaminergic treatment. Neurol. Sci. Neurophysiol. 37, 11-17 (2020).

50. Drew, D. S. et al. Dopamine and reward hypersensitivity in Parkinson's disease with impulse control disorder. Brain 143, $2502-2518$ (2020).

51. A.H., E. et al. Compulsive use of dopaminergic drug therapy in Parkinson's disease: reward and anti-reward. Mov. Disord. 25, 867-876 (2010).

52. Lawrence, A. D., Goerendt, I. K., Brooks, D. J., A.D., L. \& I.K., G. Apathy blunts neural response to money in Parkinson's disease. Soc. Neurosci. 6, 653-662 (2011).

53. Muhammed, K. et al. Reward sensitivity deficits modulated by dopamine are associated with apathy in Parkinson's disease. Brain 139, 27062721 (2016).

54. J.B., R. et al. Motivational engagement in Parkinson's disease: Preparation for motivated action. Int. J. Psychophysiol. 99, 24-32 (2016).

55. M.H.M., T. et al. Enhanced motivation of cognitive control in Parkinson's disease. Eur. J. Neurosci. 48, $2374-2384$ (2018). 
Supplementary references for Table 1.

s1. den Brok, M. G. H. E. et al. Apathy in Parkinson's disease: A systematic review and meta-analysis. Mov. Disord. (2015). doi:10.1002/mds.26208

s2. Lemke, M. R. Anhedonia, Depression, and Motor Functioning in Parkinson's Disease During Treatment With Pramipexole. J. Neuropsychiatr. (2005). doi:10.1176/appi.neuropsych.17.2.214

s3. Husain, M. \& Roiser, J. P. Neuroscience of apathy and anhedonia: A transdiagnostic approach. Nature Reviews Neuroscience (2018). doi:10.1038/s41583-018-0029-9

s4. Reijnders, J. S. A. M., Ehrt, U., Weber, W. E. J., Aarsland, D. \& Leentjens, A. F. G. A systematic review of prevalence studies of depression in Parkinson's disease. Movement Disorders (2008). doi:10.1002/mds.21803

s5. Schrag, A., Ben-Shlomo, Y. \& Quinn, N. How common are complications of Parkinson's disease? J. Neurol. 249, 419-423 (2002).

s6. Halahakoon, D. C. et al. Reward-Processing Behavior in Depressed Participants Relative to Healthy Volunteers: A Systematic Review and Meta-analysis. JAMA Psychiatry (2020). doi:10.1001/jamapsychiatry.2020.2139

s7. Broen, M. P. G., Narayen, N. E., Kuijf, M. L., Dissanayaka, N. N. W. \& Leentjens, A. F. G. Prevalence of anxiety in Parkinson's disease: A systematic review and meta-analysis. Mov. Disord. 31, 1125-1133 (2016).

s8. $\quad$ Charpentier, C. J., Aylward, J., Roiser, J. P. \& Robinson, O. J. Enhanced Risk Aversion, But Not Loss Aversion, in Unmedicated Pathological Anxiety. Biol. Psychiatry 81, 1014-1022 (2017).

s9. Drew, D. S. et al. Dopamine and reward hypersensitivity in Parkinson's disease with I mpulse control disorder. Brain 143, 2502-2518 (2020).

s10.. Pezzella, F. R. et al. Prevalence and clinical features of hedonistic homeostatic dysregulation in Parkinson's disease. Mov. Disord. 20, 77-81 (2005).

s11. Luijten, M., Schellekens, A. F., Kühn, S., Machielse, M. W. J. \& Sescousse, G. Disruption of Reward Processing in Addiction: An Image-Based Meta-analysis of Functional Magnetic Resonance Imaging Studies. JAMA Psychiatry 74, 387-398 (2017).

s12. Fénelon, G. \& Alves, G. Epidemiology of psychosis in Parkinson's disease. J. Neurol. Sci. 289, 12-17 (2010).

S13. Radua, J. et al. Ventral Striatal Activation During Reward Processing in Psychosis: A Neurofunctional Meta-Analysis. JAMA Psychiatry 72, 1243-1251 (2015). 


\section{Supplement figure 1. Contour-Enhanced Funnel Plot of all studies Parkinson's versus healthy controls}

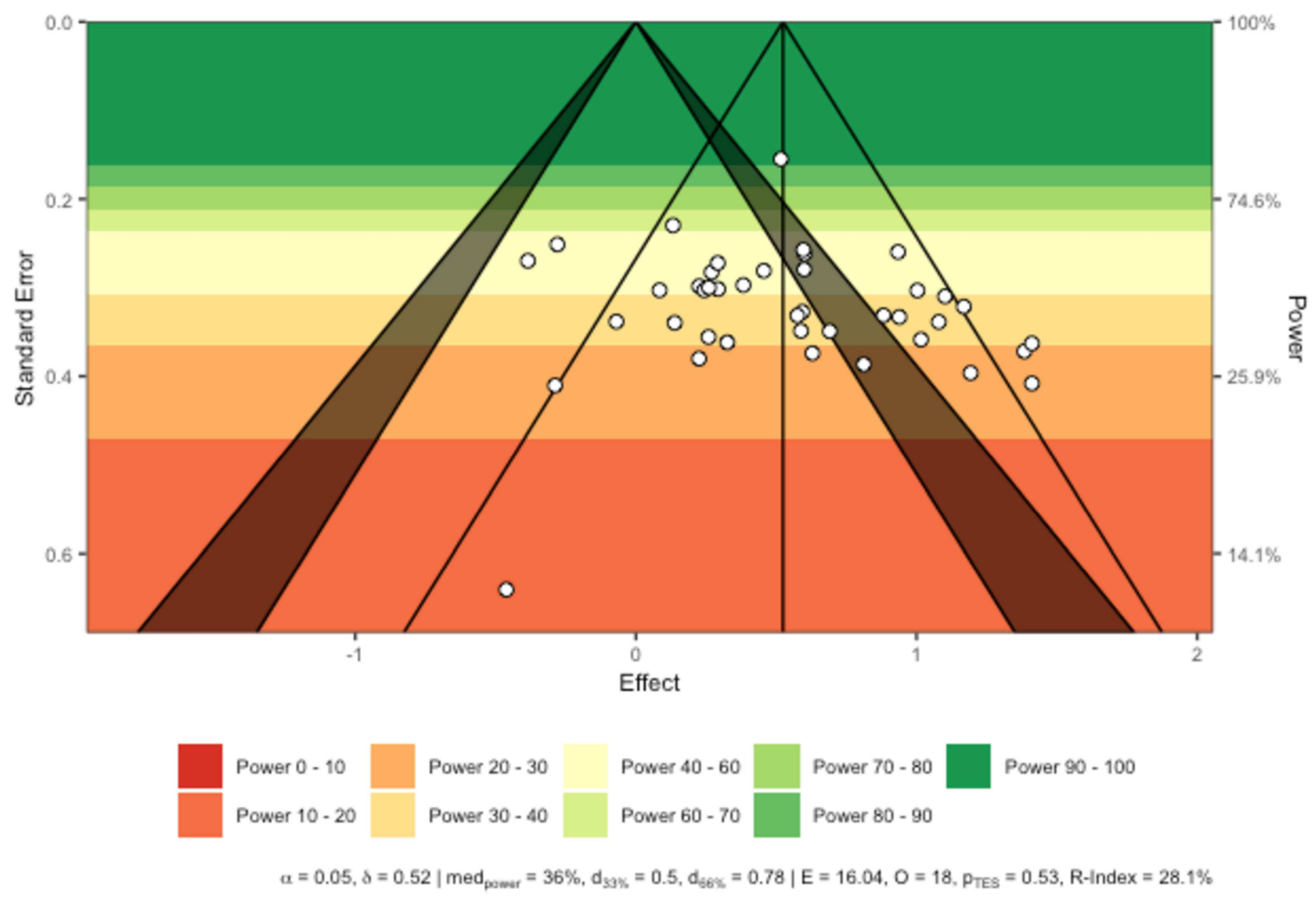

Supplement figure 2. Forest plot of reinforcement learning (RL) in Parkinson's patients with and without Impulse Control Disorder (ICD)

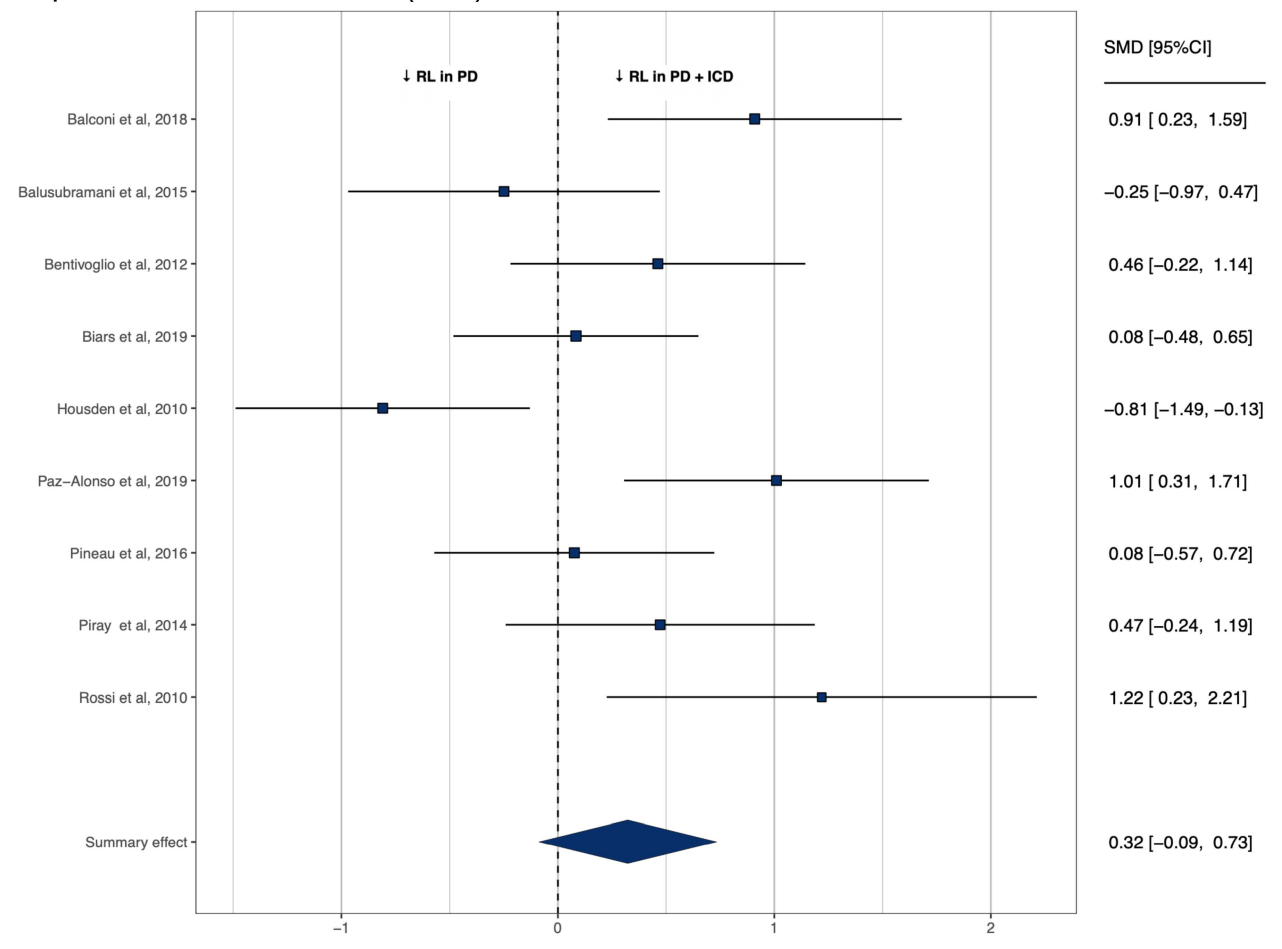




\section{Formulae used to convert study measures into Cohen's ds and associated variances.}

The following formulae were used to convert study measures into Cohen's ds and associated variances between subjects:

$$
d=\frac{M_{1}-M_{2}}{S D_{\text {pooled }}}
$$

Equation 1. Cohen's d from Means and Standard Deviations of 2 samples. $d$ is Cohen's d, M1 is the mean of one sample M2 is the mean of the other sample, SDpooled is the pooled standard deviation of the two samples (please see below.)

$$
S D_{\text {pooled }}=\sqrt{\left(N_{1}-1\right) S D_{1}^{2}+\left(N_{2}-1\right) S D_{2}^{2}}
$$

Equation 2. Pooled standard deviation of 2 samples. SDpooled is the pooled standard deviation of the two samples, N1 is the size of one sample N2 is the size of the other sample, SD1 is the standard deviation of one sample, SD2 is the standard deviation of the other sample.

$$
d=\frac{t}{\sqrt{\frac{1}{N_{1}}+\frac{1}{N_{2}}}}
$$

Equation 3. Cohen's d from t-statistic. $d$ is Cohen's d, N1 is the size of one sample N2 is the size of the other sample, $t$ is the tstatistic

$$
d=\frac{F}{\sqrt{\frac{1}{N_{1}}+\frac{1}{N_{2}}}}
$$

Equation 4. Cohen's d from F-statistic. $d$ is Cohen's d, N1 is the size of one sample N2 is the size of the other sample, F is the Fstatistic.

$$
\operatorname{Var}_{d}=\frac{N_{1}+N_{2}}{N_{1} \times N_{2}}+\frac{d^{2}}{2\left(N_{1}+N_{2}\right)}
$$

Equation 5. Variance on Cohen's d for between subjects. Vard is the Variance on Cohen's d, N1 is the size of one sample N2 is the size of the other sample, $d$ is Cohen's $d$.

The following formulae were used to convert study measures into Cohen's ds and associated variances within subjects:

$$
d=\frac{M_{d}}{S D_{d}}
$$

Equation 6. Cohen's d from Means and Standard Deviations from within subjects sample. Where $M_{d}$ is the mean change and $S D_{d}$ is the $\mathrm{SD}$ of the change scores

(equal to $S D_{d}=\sqrt{S D_{1}^{2}+S D_{2}^{2}-2 \times r \times S D_{1} S D_{2}}$ ).

$$
d=\frac{t}{\sqrt{N}}
$$

Equation 7. Cohen's d from t-statistic for within subjects results. $\mathrm{d}$ is Cohen's $\mathrm{d}, \mathrm{N}$ is the sample size, $\mathrm{t}$ is the t-statistic

$$
d=\sqrt{\frac{F}{N}}
$$

Equation 8. Cohen's d from F-statistic. $d$ is Cohen's d, $N$ is the sample size, $\mathrm{F}$ is the F-statistic.

$$
\operatorname{Var}[d]=\frac{1}{n}+\frac{d^{2}}{2 n}
$$

Equation 9. Variance on Cohen's d for within subjects, $\mathrm{n}$ is the size of the sample. 
Supplement table 1. Option Valuation study characteristics and participant demographics.

\begin{tabular}{|c|c|c|c|c|c|c|c|c|c|c|c|c|c|c|c|c|}
\hline \multicolumn{4}{|c|}{ Option valuation in Parkinson's vs Healthy Controls } & \multicolumn{2}{|c|}{ Age (years) } & \multirow[b]{2}{*}{$\begin{array}{c}\% \\
\text { female }\end{array}$} & \multicolumn{2}{|c|}{ Disease duration (years) } & \multicolumn{2}{|c|}{ UPDRS } & \multicolumn{3}{|c|}{ Cognition } & \multicolumn{3}{|c|}{ Depression } \\
\hline Study & Task & Group & $\mathbf{n}$ & Mean & SD & & Mean & SD & Mean & SD & $\begin{array}{l}\text { Cognitive } \\
\text { measure }\end{array}$ & Mean & SD & $\begin{array}{c}\text { Depression } \\
\text { measure }\end{array}$ & Mean & SD \\
\hline \multirow{2}{*}{$\begin{array}{l}\text { Bayard, } \\
2016\end{array}$} & \multirow{2}{*}{ Game of dice task } & PD & 78 & 67.49 & 8.16 & 35 & 7 & . & 24 & $(5-80)$ & MMSE & 28.1 & 1.93 & $\mathrm{BDI}$ & 12.5 & 7.38 \\
\hline & & $\mathrm{HC}$ & 96 & 67.95 & 6.75 & 32 & - & - & - & - & & 28.46 & 1.38 & & 10.04 & 11.76 \\
\hline \multirow{2}{*}{$\begin{array}{l}\text { Brandt, } \\
2015\end{array}$} & \multirow{2}{*}{ Game of dice task } & PD & 15 & 64.78 & 8.09 & 46.66 & . & . & 14.43 & 10.14 & MoCA & 26.87 & 1.69 & GDS-15 & 3.33 & 3.54 \\
\hline & & $\mathrm{HC}$ & 15 & 62.39 & 10.04 & 40 & - & - & - & - & & 27.6 & 1.43 & & 1.33 & 1.18 \\
\hline \multirow{2}{*}{$\begin{array}{l}\text { Chong, } \\
2015\end{array}$} & \multirow{2}{*}{$\begin{array}{l}\text { Effort based decision } \\
\text { making task }\end{array}$} & PD & 26 & 66.6 & 6.8 & 34.6 & . & . & 21.6 & 11.7 & MoCA & 28.2 & 1.3 & DASS & 2 & 2.23 \\
\hline & & $\mathrm{HC}$ & 26 & 66.2 & 6.4 & 42.3 & - & - & - & - & & 28.2 & 1.7 & & 1.5 & 1.84 \\
\hline \multirow{2}{*}{ Cools, 2003} & \multirow{2}{*}{$\begin{array}{l}\text { Decision making } \\
\text { gambling task }\end{array}$} & PD & 12 & 64.6 & 1.5 & 58.33 & 6.5 & 1.4 & 30.9 & 6.8 & MMSE & 29.5 & 0.15 & $\mathrm{BDI}$ & 7.7 & 1.1 \\
\hline & & $\mathrm{HC}$ & 12 & & . & & - & - & - & - & & & & & . & \\
\hline \multirow{2}{*}{$\begin{array}{l}\text { Kobayashi, } \\
2019\end{array}$} & \multirow{2}{*}{ Economic choice task } & $\mathrm{PD}$ & 15 & 63 & 6.58 & 40 & 6.93 & 6.2 & 39 & 14.6 & MMSE & 28.3 & 1.76 & . & . & . \\
\hline & & $\mathrm{HC}$ & 21 & 60 & 6.69 & 57.1 & - & - & - & - & & 28.7 & 1.99 & & . & . \\
\hline \multirow{2}{*}{$\begin{array}{l}\text { Le Bouc, } \\
2016\end{array}$} & \multirow{2}{*}{$\begin{array}{l}\text { Incentivised grip } \\
\text { force choice task }\end{array}$} & $\mathrm{PD}$ & 24 & 60.2 & 1.6 & 29.17 & 11.4 & 1.3 & 11.7 & 1.7 & MMSE & 27.3 & 1.3 & MADRS & 5.9 & 1 \\
\hline & & $\mathrm{HC}$ & 25 & 57 & 2.1 & 52 & - & - & - & - & & . & . & & 2.9 & 0.7 \\
\hline \multirow{2}{*}{$\begin{array}{l}\text { Le Heron, } \\
2018\end{array}$} & \multirow{2}{*}{$\begin{array}{l}\text { Effort based decision } \\
\text { making task }\end{array}$} & $\mathrm{PD}$ & 39 & 67.8 & 7.6 & 19.04 & . & . & 29.5 & 9.9 & ACE & 91.1 & 7.9 & $\mathrm{BDI}$ & 14.3 & 7.7 \\
\hline & & $\mathrm{HC}$ & 32 & 68.9 & 6.9 & 40.6 & - & - & - & - & & 95.6 & 3.8 & & 3.8 & 3.7 \\
\hline \multirow{2}{*}{$\begin{array}{l}\text { Mcguigan, } \\
2018\end{array}$} & \multirow{2}{*}{ Cognitive effort task } & PD & 20 & 67.1 & 9.1 & 40 & 5.4 & 4.9 & 27.3 & 18.1 & MoCA & 27.7 & 1.92 & $\mathrm{BDI}$ & 9.5 & 4.98 \\
\hline & & $\mathrm{HC}$ & 20 & 61.1 & 13.6 & 40 & - & - & - & - & & 28.1 & 1.37 & & 3.55 & 4.13 \\
\hline \multirow{2}{*}{ Torta, 2009} & \multirow{2}{*}{$\begin{array}{l}\text { Cambridge gamble } \\
\text { task }\end{array}$} & PD & 15 & 58.4 & 6.9 & 13.33 & 13.2 & 3.2 & 16.52 & 7.5 & MMSE & 28.2 & 1.5 & $\mathrm{BDI}$ & 10 & 6.6 \\
\hline & & $\mathrm{HC}$ & 13 & 58.2 & 5.7 & 15.38 & - & - & - & - & & & . & & . & . \\
\hline \multirow{2}{*}{$\begin{array}{l}\text { Sharp, } \\
2013\end{array}$} & Vancouver gambling & $\mathrm{PD}$ & 18 & 65.47 & 9.17 & 27.78 & 5.59 & 4.04 & 20.65 & 6.91 & MOCA & 27.94 & 1.14 & $\mathrm{BDI}$ & 6.06 & 3.78 \\
\hline & task (modified) & $\mathrm{HC}$ & 18 & 66.76 & 5.83 & 50 & - & - & - & & & 28.85 & 1.32 & & 5.18 & 3.57 \\
\hline Option va & $\begin{array}{l}\text { ation in Parkinson's wi } \\
\text { syndrome vs without }\end{array}$ & psychiat & & Age ( & ears) & & Disease dur & & UP & & & gnition & & Dep & ression & \\
\hline Study & Task & Group & $\mathrm{n}$ & Mean & SD & $\begin{array}{c}\% \\
\text { female }\end{array}$ & Mean & SD & Mean & SD & $\begin{array}{l}\text { Cognitive } \\
\text { measure }\end{array}$ & Mean & SD & $\begin{array}{c}\text { Depression } \\
\text { measure }\end{array}$ & Mean & SD \\
\hline Haagensen, & Game of dice task & $\mathrm{PD}$ & 13 & 61.4 & 9.7 & 46.15 & 4.5 & 2 & 22.8 & 6.9 & MoCA & 28.7 & 1.3 & $\mathrm{BDI}$ & 7 & 5 \\
\hline 2020 & & $\mathrm{ICD}$ & 13 & 59.4 & 10.9 & 38.46 & 6.5 & 3.6 & 22.6 & 6 & & 28.7 & 0.9 & & 7.4 & 6.5 \\
\hline Kobayashi, & Eronomic choire tack & $\mathrm{PD}$ & 15 & 63 & 6.58 & 40 & 6.93 & 6.2 & 39 & 14.6 & MMSE & 28.3 & 1.76 & . & . & \\
\hline 2019 & 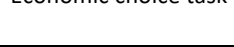 & $I C D$ & 10 & 63.1 & 9.68 & 40 & 8.4 & 2.99 & 49.9 & 26.3 & & 28.3 & 1.77 & $\cdot$ & . & $\cdot$ \\
\hline Le Heron, & Effort based decision & $\mathrm{PD}$ & 18 & 68.2 & 6.5 & 44.44 & . & . & 27.1 & 13.2 & ACE & 93.5 & 5 & $\mathrm{BDI}$ & 11 & 7 \\
\hline 2018 & & Apathy & 21 & 67.5 & 8.5 & 19.05 & & & 29.5 & 9.9 & & 89.4 & 9.4 & & 17.1 & 7.4 \\
\hline
\end{tabular}


\begin{tabular}{|l|l|c|c|c|c|c|}
\multirow{3}{*}{ Voon, 2011 } & \multirow{3}{*}{ Gambling task } & PD & 14 & 54.5 & 12.5 & 71.4 \\
\cline { 2 - 7 } & & ICD & 14 & 51.5 & 8.3 & 71.4 \\
\hline
\end{tabular}

$P D=$ Parkinson's disease, $H C=$ healthy controls, ICD = Impulse control disorder, $S D=$ standard deviation, IQR = interquartile range, ' $. '=$ not reported, ' - ' = not applicable, UPDRS = Unified Parkinson's Disease Rating Scale, MMSE = Mini-mental state examination, MoCA = Montreal cognitive assessment, ACE = Addenbrooke's cognitive examination, BDI $=$ Beck depression inventory, DASS $=$ Depression, Anxiety and Stress Scale, GDS = Geriatric depression scale, MADRS = Montgomery-Asberg Depression Rating Scale

Supplement table 2. Reinforcement Learning study characteristics and participant demographics.

\begin{tabular}{|c|c|c|c|c|c|c|c|c|c|c|c|c|c|c|c|c|}
\hline \multicolumn{4}{|c|}{ Reinforcement learning in Parkinson's vs Healthy Controls } & \multicolumn{2}{|c|}{ Age (years) } & \multirow[b]{2}{*}{$\begin{array}{c}\% \\
\text { female }\end{array}$} & \multicolumn{2}{|c|}{ Disease duration (years) } & \multicolumn{2}{|c|}{ UPDRS } & \multicolumn{3}{|c|}{ Cognition } & \multicolumn{3}{|c|}{ Depression } \\
\hline Study & Task & Group & $\mathrm{n}$ & Mean & $\begin{array}{l}\text { SD or } \\
\text { (IQR) }\end{array}$ & & Mean & $\begin{array}{l}\text { SD or } \\
\text { (IQR) }\end{array}$ & Mean & $\begin{array}{l}\text { SD or } \\
\text { (IQR) }\end{array}$ & Measure & Mean & $\begin{array}{l}\text { SD or } \\
\text { (IQR) }\end{array}$ & Measure & Mean & $\begin{array}{l}\text { SD or } \\
\text { (IQR) }\end{array}$ \\
\hline \multirow{2}{*}{ Castrioto, 2015} & \multirow{2}{*}{ IGT } & PD & 20 & 53.2 & 6.6 & 45 & 10.3 & 3.8 & 12.3 & 6.1 & MDRS & 137.6 & 4.2 & . & . & . \\
\hline & & $\mathrm{HC}$ & 24 & 54.9 & 7.6 & 62.5 & - & - & - & - & & 140.7 & 2.4 & & . & . \\
\hline \multirow{2}{*}{$\begin{array}{l}\text { Balusubramani, } \\
\quad 2015\end{array}$} & \multirow{2}{*}{$\begin{array}{c}\text { Probabilistic } \\
\text { rewarded } \\
\text { categorisation } \\
\text { learning task }\end{array}$} & PD & 30 & . & . & 18.75 & 9.5 & . & . & . & MMSE & . & . & $\mathrm{BDI}$ & . & . \\
\hline & & $\mathrm{HC}$ & 20 & & . & 13.04 & - & - & - & - & & & & & 5 & 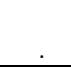 \\
\hline \multirow{2}{*}{ Bodi, 2009} & \multirow{2}{*}{$\begin{array}{c}\text { Probabilistic } \\
\text { classification task }\end{array}$} & PD & 22 & 44.8 & 5.2 & 30.77 & 0.266 & 0.166 & 27.5 & 6.1 & . & . & . & HAM-D & 4.2 & 1.4 \\
\hline & & $\mathrm{HC}$ & 20 & 45.3 & 8.5 & 25 & - & - & - & - & & . & . & & . & . \\
\hline \multirow{2}{*}{ Buelow, 2014} & \multirow{2}{*}{ IGT } & $\mathrm{PD}$ & 24 & 68.04 & 7.86 & 45.8 & . & . & 29.89 & 13.14 & MMSE & 28.33 & 1.63 & GDS & 2.5 & 1.91 \\
\hline & & $\mathrm{HC}$ & 14 & 69.62 & 6.36 & 53.85 & - & - & - & - & & 29.31 & 1.11 & & 1.15 & 1.57 \\
\hline \multirow{2}{*}{$\begin{array}{c}\text { Cavanagh, } \\
2017\end{array}$} & \multirow{2}{*}{ Cost of conflict task } & $\mathrm{PD}$ & 28 & 69.75 & 8.59 & 39.28 & 5.54 & 4.18 & & & MMSE & 28.64 & 1.06 & $\mathrm{BDI}$ & 7.64 & 5.23 \\
\hline & & $\mathrm{HC}$ & 28 & 69.21 & 9.23 & 39.28 & - & - & - & - & & 28.82 & 1.02 & & 4.93 & 4.69 \\
\hline \multirow{2}{*}{$\begin{array}{l}\text { Czernecki, } \\
2002\end{array}$} & \multirow{2}{*}{ IGT } & PD & 23 & 57.6 & 2.1 & 60.87 & 14.9 & 1.2 & 12.4 & 2 & MDRS & 139.1 & 0.8 & MADRS & 8.3 & 1.4 \\
\hline & & $\mathrm{HC}$ & 28 & 58.1 & 1.7 & 35.71 & - & - & - & - & & 141.1 & 0.3 & & 6.2 & 0.8 \\
\hline \multirow{2}{*}{ Delazer, 2009} & \multirow{2}{*}{ IGT } & $\mathrm{PD}$ & 20 & 68.5 & 5.9 & 25 & 5.25 & 6.38 & 17.6 & 8.7 & MMSE & 27.8 & 1.9 & HADS & 6.6 & 3.2 \\
\hline & & $\mathrm{HC}$ & 20 & 71.3 & 3.5 & 85 & - & - & - & - & & 29.8 & 0.4 & & . & . \\
\hline \multirow{2}{*}{$\begin{array}{l}\text { Euteneuer, } \\
2009\end{array}$} & \multirow{2}{*}{ IGT } & PD & 21 & 67.6 & 7.31 & 67 & 7.14 & 6.06 & 17.7 & 9.2 & MMSE & 29 & 1.1 & $\mathrm{BDI}$ & 3.9 & 2.12 \\
\hline & & $\mathrm{HC}$ & 23 & 64.4 & 8.56 & 48 & & & & & & 29.65 & 0.65 & & 0.83 & 1.3 \\
\hline \multirow{2}{*}{ Evens, 2015} & \multirow{2}{*}{ IGT } & PD & 32 & 65.12 & 8.25 & 25 & 7.38 & 4.33 & 16.58 & 7.36 & MMSE & 29.25 & 0.98 & MADRS & 4.33 & 3.68 \\
\hline & & $\mathrm{HC}$ & 32 & 65.53 & 5.94 & 31 & - & - & - & - & & 29.34 & 0.83 & & 1.59 & 2.19 \\
\hline \multirow{2}{*}{ Garofalo, 2017} & \multirow{2}{*}{$\begin{array}{c}\text { Instrumental } \\
\text { conditioning task }\end{array}$} & PD & 17 & 63.29 & 9.94 & 50 & 16.42 & 28.77 & . & . & MMSE & 28.41 & 1.37 & $\mathrm{BDI}$ & 12.66 & 7.83 \\
\hline & & $\mathrm{HC}$ & 24 & 61.91 & 5.83 & 44 & - & - & - & - & & 28.94 & 1.54 & & 8.88 & 4.94 \\
\hline \multirow{2}{*}{$\begin{array}{l}\text { Gescheidt, } \\
2012\end{array}$} & IGT & PD & 19 (early onset) & 50.32 & 8.74 & 25 & 11.32 & 6.42 & 14.6 & 8.7 & MMSE & 29.37 & 0.96 & MADRS & . & . \\
\hline & & $\mathrm{HC}$ & 20 & 49.95 & 9.03 & 26.3 & - & - & - & - & & 29.7 & 0.47 & & . & . \\
\hline Graef, 2010 & & PD & 15 & 65.27 & 8.14 & 40 & 4.3 & 4 & 19.64 & 7.7 & MMSE & 29 & 1 & $\mathrm{BDI}$ & 6.93 & 5.55 \\
\hline
\end{tabular}




\begin{tabular}{|c|c|c|c|c|c|c|c|c|c|c|c|c|c|c|c|c|}
\hline & $\begin{array}{c}\text { Probabilistic } \\
\text { reversal learning } \\
\text { task }\end{array}$ & $\mathrm{HC}$ & 16 & 67.75 & 4.55 & 37.5 & - & - & - & - & & 28.64 & 1.15 & & 5.31 & 2.98 \\
\hline \multirow{2}{*}{ Herzallah, 2017} & \multirow{2}{*}{$\begin{array}{l}\text { Rewarded } \\
\text { categorisation task }\end{array}$} & PD & 17 & 59.4 & 12.6 & 11.76 & 5.24 & 4 & 28.6 & 14.6 & MMSE & 28.5 & 1.1 & $\mathrm{BDI}$ & 8.3 & 5.2 \\
\hline & & $\mathrm{HC}$ & 15 & 54.3 & 12.3 & 33.33 & - & - & - & - & & 29.3 & 0.7 & & 6.7 & 5.3 \\
\hline \multirow{2}{*}{ Housden, 2010} & \multirow{2}{*}{$\begin{array}{l}\text { Rewarded salience } \\
\text { attribution test }\end{array}$} & PD & 18 & 67.7 & 5.5 & 38.89 & 12.9 & 8.3 & 20 & 6.6 & MMSE & 28.6 & 2.1 & $\mathrm{BDI}$ & 12.9 & 9.9 \\
\hline & & $\mathrm{HC}$ & 20 & 65.5 & 6 & 33.33 & - & - & - & - & & 29.4 & 0.8 & & 11.3 & 6.9 \\
\hline \multirow{2}{*}{$\begin{array}{l}\text { Ibarretxe- } \\
\text { Bilbao, } 2009\end{array}$} & \multirow{2}{*}{ IGT } & PD & 24 & 56.13 & 8.5 & 33 & 3.06 & 1.6 & 14.67 & 3.5 & MMSE & 29.63 & 0.5 & $\mathrm{BDI}$ & $\cdot$ & . \\
\hline & & $\mathrm{HC}$ & 24 & 57.58 & 8.9 & 33 & - & - & - & - & & 29.83 & 0.4 & & & \\
\hline \multirow{2}{*}{$\begin{array}{l}\text { Kobayakawa, } \\
2008\end{array}$} & \multirow{2}{*}{ IGT } & $\mathrm{PD}$ & 34 & 69.9 & 8.9 & 64.7 & 6.4 & 3.4 & . & . & MMSE & 28 & 2.2 & SDS & 36.1 & 8.9 \\
\hline & & $\mathrm{HC}$ & 22 & 67.6 & 6.9 & 40.9 & - & - & - & - & & 28.8 & 1.6 & & 29.1 & 6 \\
\hline \multirow{2}{*}{$\begin{array}{l}\text { Kobayakawa, } \\
2010\end{array}$} & \multirow{2}{*}{ IGT } & $\mathrm{PD}$ & 14 & 68.9 & 8 & 50 & 5.6 & 2.7 & . & . & MMSE & 28.2 & 1.9 & SDS & 32.9 & 7.8 \\
\hline & & $\mathrm{HC}$ & 22 & 67.6 & 6.9 & 69.2 & - & - & - & - & & 28.8 & 1.6 & & 29.1 & 6 \\
\hline \multirow{2}{*}{ Mapelli, 2014} & \multirow{2}{*}{ IGT } & $\mathrm{PD}$ & 15 & 61.4 & 9.6 & 25 & 4.8 & 3.4 & 8.9 & 4 & MMSE & 28.3 & 1.2 & $\mathrm{BDI}$ & \multicolumn{2}{|c|}{ Excluded if $>14$} \\
\hline & & $\mathrm{HC}$ & 15 & 60.7 & 9.8 & 26.7 & - & - & - & - & & 27.86 & 1.5 & & . & . \\
\hline \multirow{2}{*}{ Mimura, 2006} & \multirow{2}{*}{ IGT } & $\mathrm{PD}$ & 18 & 68.9 & 7 & 72.2 & . & . & . & . & MMSE & 27.8 & 1.9 & ZSRD & 39.4 & 6.9 \\
\hline & & $\mathrm{HC}$ & 20 & . & . & 70 & - & - & - & - & & 29.1 & 1.5 & & 30.5 & 6 \\
\hline \multirow{2}{*}{$\begin{array}{c}\text { Pagonabarraga, } \\
2007\end{array}$} & \multirow{2}{*}{ IGT } & PD & 35 & 67.2 & 8 & 37.2 & 8.4 & 5 & 21.2 & 8 & MDRS & 133 & 6 & & . & . \\
\hline & & $\mathrm{HC}$ & 31 & 70.2 & 10 & 47.7 & - & - & - & - & & 136 & 5 & & . & . \\
\hline \multirow{2}{*}{$\begin{array}{l}\text { Paz-Alonso, } \\
2019\end{array}$} & \multirow{2}{*}{ IGT } & $\mathrm{PD}$ & 17 & 61 & 8.7 & 11.8 & 7 & $(4-10)$ & 25.9 & 8.2 & . & & & HADS-D & 2.2 & 2.6 \\
\hline & & $\mathrm{HC}$ & 18 & 63 & 9.7 & 16.7 & - & - & - & - & & & & & 1.9 & 1.3 \\
\hline \multirow{3}{*}{ Perretta, 2005} & \multirow{3}{*}{ IGT } & PD (late stage) & 16 & 77.7 & 6 & 50 & . & . & 27.2 & 1.3 & MMSE & Excluded if $<27$ & . & $\mathrm{BDI}$ & 12.7 & 1.5 \\
\hline & & PD (early stage) & 16 & 72.4 & 2.3 & 43.75 & . & & 11.3 & 1.1 & & & & & 6.9 & 0.7 \\
\hline & & $\mathrm{HC}$ & 19 & 72.6 & 8.28 & 42.1 & - & - & - & - & & & & & 5.2 & 0.8 \\
\hline \multirow{2}{*}{ Pignatti, 2012} & \multirow{2}{*}{ IGT } & $\mathrm{PD}$ & 15 & 64.27 & 10.5 & . & . & . & . & . & MMSE & 28.31 & 1.11 & . & . & . \\
\hline & & $\mathrm{HC}$ & 16 & 41.56 & 13.9 & . & - & - & - & - & & & & & . & . \\
\hline \multirow{2}{*}{ Piray, 2014} & \multirow{2}{*}{$\begin{array}{c}\text { Rewarded } \\
\text { categorisation task }\end{array}$} & $\mathrm{PD}$ & 40 & 63.33 & 3.98 & 23.07 & 9.72 & 2.64 & 19.6 & 6.42 & MMSE & 27 & 0.93 & $\mathrm{BDI}$ & 8 & 1.69 \\
\hline & & $\mathrm{HC}$ & 20 & 66.45 & 4.7 & 35 & - & - & - & - & & 27.65 & 1.18 & & 7.75 & 1.97 \\
\hline Poletti, 2010 & IGT & PD (off) & 24 & 64.9 & 5.8 & 27 & 0 (de novo) & & 14.3 & 8.1 & MMSE & 28.8 & 2 & GDS & 4.7 & 3.3 \\
\hline & & $\mathrm{HC}$ & 25 & 65.4 & 2.2 & 44 & & & & & & 28.5 & 1.8 & & 2.8 & 1.4 \\
\hline Thiel 2003 & JGT & $\mathrm{PD}$ & 5 & 62.6 & 12.5 & 40 & 8 & 2.55 & 20.6 & 6.47 & MMSE & .28 .4 & 2.07 & & . & . \\
\hline & 然 & $\mathrm{HC}$ & 5 & 44.2 & 21.3 & 20 & - & - & - & - & & & & & & \\
\hline $\mathrm{Xi}, 2015$ & IGT & PD & 15 & 60.73 & 11.7 & 53 & 4.33 & 5.05 & 15.87 & 8.96 & MMSE & 27.6 & 4.63 & HAMD & 4.67 & 1.29 \\
\hline
\end{tabular}




\begin{tabular}{|c|c|c|c|c|c|c|c|c|c|c|c|c|c|c|c|c|}
\hline & & $\mathrm{HC}$ & 15 & 56.33 & 14.5 & 60 & - & - & - & - & & 29.4 & 1.12 & & 3.8 & 1.37 \\
\hline \multirow{2}{*}{ Yildirim, 2020} & \multirow{2}{*}{ IGT } & PD & 39 & 65.9 & 7.3 & 21 & 7.8 & 4.3 & 11.6 & 6.1 & MMSE & . & . & . & . & . \\
\hline & & $\mathrm{HC}$ & 37 & 64.4 & 8 & 24 & - & - & - & - & & & . & & . & . \\
\hline \multicolumn{4}{|c|}{$\begin{array}{l}\text { Reinforcement learning in Parkinson's with psychiatric syndrome vs } \\
\text { without }\end{array}$} & \multicolumn{2}{|c|}{ Age } & & \multicolumn{2}{|c|}{ Disease duration (years) } & \multicolumn{2}{|c|}{ UPDRS } & \multicolumn{3}{|c|}{ Cognition } & & \multicolumn{2}{|c|}{ Depression } \\
\hline Study & Task & Group & $\mathrm{n}$ & Mean & $\begin{array}{l}\text { SD or } \\
\text { (IQR) }\end{array}$ & $\begin{array}{c}\% \\
\text { female }\end{array}$ & Mean & $\begin{array}{l}\text { SD or } \\
\text { (IQR) }\end{array}$ & Mean & $\begin{array}{l}\text { SD or } \\
\text { (IQR) }\end{array}$ & Measure & Mean & $\begin{array}{l}\text { SD or } \\
\text { (IQR) }\end{array}$ & $\begin{array}{c}\text { Depression } \\
\text { measure }\end{array}$ & Mean & $\begin{array}{l}\text { SD or } \\
\text { (IQR) }\end{array}$ \\
\hline \multirow{2}{*}{ Balconi, 2018} & \multirow{2}{*}{ IGT } & PD & 20 & 63.9 & 7.1 & 15 & & & 13 & 9.3 & & & . & $\mathrm{BDI}$ & 11.3 & 6 \\
\hline & & PG & 17 & 60.7 & 6.1 & 17.65 & . & & 17 & 7.8 & & . & . & & 15.9 & 9.1 \\
\hline \multirow{2}{*}{$\begin{array}{l}\text { Balusubramani, } \\
\quad 2015\end{array}$} & \multirow{2}{*}{$\begin{array}{l}\text { Rewarded } \\
\text { categorisation task }\end{array}$} & PD & 14 & . & . & 21.4 & 8.35 & & . & . & MMSE & . & . & $\mathrm{BDI}$ & . & . \\
\hline & & $\mathrm{ICD}$ & 16 & 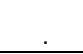 & & 12.5 & 9.56 & & & & & & & & & \\
\hline \multirow{2}{*}{$\begin{array}{l}\text { Bentivoglio, } \\
2012\end{array}$} & \multirow{2}{*}{ IGT } & PD & 17 & 63.94 & 9.2 & 35.29 & 7.3 & 4.4 & 22.5 & 6.9 & MMSE & 28.6 & 1.4 & HAM-D & 5.6 & 4.1 \\
\hline & & ICD & 17 & 62 & 10.1 & 17.65 & 6.9 & 3.8 & 23.8 & 11 & & 28.4 & 1.6 & & 7.5 & 6.3 \\
\hline \multirow{2}{*}{ Biars, 2019} & \multirow{2}{*}{ IGT } & $\mathrm{PD}$ & 24 & 60.5 & 7.8 & 12.5 & 11.9 & 7.1 & 37.2 & 14.8 & DRS & 136.5 & 5.9 & $\mathrm{BDI}$ & 8.5 & 5.3 \\
\hline & & $\mathrm{ICD}$ & 24 & 61.2 & 8.3 & 12.5 & 13.2 & 7.1 & 21.6 & 9.9 & & 138 & 3.5 & & 11.2 & 6.8 \\
\hline \multirow{2}{*}{ Buelow, 2014} & \multirow{2}{*}{ IGT } & PD & 14 & 69 & 6.19 & 57.14 & . & & 29.62 & 6.85 & MMSE & 28.71 & 1.38 & GDS & 2.14 & 1.41 \\
\hline & & Apathy & 10 & 66.7 & 9.96 & 30 & . & & 29.89 & 13.14 & & 27.8 & 1.87 & & 3 & 2.45 \\
\hline \multirow{2}{*}{ Garofalo, 2017} & \multirow{2}{*}{$\begin{array}{l}\text { Instrumental } \\
\text { conditioning task }\end{array}$} & PD & 17 & 63.29 & 9.94 & 44 & 10.94 & 10.38 & . & . & MMSE & 28.94 & 1.54 & $\mathrm{BDI}$ & 8.88 & 4.94 \\
\hline & & Psychosis & 12 & 60.83 & 6.6 & 50 & 16.42 & 28.77 & . & . & & 28.41 & 1.37 & & 12.66 & 7.83 \\
\hline \multirow{2}{*}{ Herzallah, 2017} & \multirow{2}{*}{$\begin{array}{c}\text { Rewarded } \\
\text { categorisation task }\end{array}$} & PD & 17 & 59.4 & 12.6 & 11.76 & 5.24 & 4 & . & . & MMSE & 28.5 & 1.1 & $\mathrm{BDI}$ & 8.3 & 5.2 \\
\hline & & $M D D$ & 13 & 55.2 & 11.9 & 69.2 & 4.84 & 3.7 & 28.6 & 14.6 & & 27.5 & 1.8 & & 26.9 & 7.7 \\
\hline \multirow{2}{*}{ Housden, 2010} & \multirow{2}{*}{$\begin{array}{c}\text { Rewarded salience } \\
\text { attribution test }\end{array}$} & PD & 18 & 67.7 & 5.5 & 33.33 & 12.9 & 8.3 & 21.3 & 0.4 & MMSE & 29.4 & 0.8 & $\mathrm{BDI}$ & 11.3 & 6.9 \\
\hline & & $I C D$ & 18 & 62.3 & 7.6 & 38.89 & 13.9 & 9 & 20 & 6.6 & & 28.6 & 2.1 & & 12.9 & 9.9 \\
\hline \multirow{2}{*}{$\begin{array}{l}\text { Martinez- } \\
\text { Horta, } 2013\end{array}$} & ITT & PD & 17 & 65.06 & 4.85 & . & 5.38 & 4.25 & 18 & 4.61 & MMSE & 28.94 & 1.24 & HADS & 3.88 & 3.6 \\
\hline & | & Apathy & 17 & 68.25 & 6.06 & . & 6.75 & 4.93 & 18.65 & 5.8 & & 28.55 & 1.23 & & 5.65 & 2.92 \\
\hline Paz-Alonso, & IGT & PD & 17 & 61 & 8.7 & 11.8 & 7 & $(4-10)$ & 25.9 & 8.2 & . & . & . & HADS & 2.2 & 2.6 \\
\hline 2019 & & $\mathrm{ICD}$ & 18 & 62.3 & 7.6 & 11.1 & 8 & $(5.1-10)$ & 22.31 & 6.6 & & . & . & & 3.1 & 2.4 \\
\hline Pineau 2016 & IGT (adanted) & PD & 20 & 55 & $(40-62)$ & 35 & 5.5 & $(4-12)$ & 8.5 & $(0-34)$ & MDRS & 139 & (131-143) & . & . & \\
\hline & & ICD & 17 & 55 & $(37-65)$ & 17.64 & 7 & $(2-10)$ & 7 & $(0-23)$ & & 140 & $(133-144)$ & & . & \\
\hline Piray 2014 & Rewarded & $\mathrm{PD}$ & 40 & 63.33 & 3.98 & 25.9 & 8.87 & 3.14 & 19.6 & 6.42 & MMSE & 27 & 0.93 & $\mathrm{BDI}$ & 8 & 1.69 \\
\hline & categorisation task & $I C D$ & 16 & 64.38 & 3.32 & 11.1 & 9.63 & 2.45 & 19 & 5.32 & & 27.19 & 1.11 & & 6.75 & 1.69 \\
\hline Dolotti 201 & GT & PD & 12 & 63.92 & 7.17 & 16.66 & $\cdot$ & & . & . & MMSE & 28.72 & 2.41 & GDS-15 & 3.33 & 2.34 \\
\hline & & Alexithymia & 12 & 66.17 & 5.2 & 41.66 & . & & . & . & & 28.49 & 1.94 & & 6.33 & 3.89 \\
\hline Rossi, 2010 & IGT & PD & 13 & 65.1 & 3.8 & 23 & & & 14.7 & 6.7 & MMSE & & . & MADRS & 14.1 & 7.9 \\
\hline
\end{tabular}




\begin{tabular}{|c|c|c|c|c|c|c|c|c|c|c|c|c|c|c|c|c|}
\hline & & PG & 7 & 61.4 & 6.9 & 14 & & . & 17 & 9.1 & & Excluded if $<24$ & . & & 17.1 & 6.5 \\
\hline \multirow{2}{*}{$\begin{array}{l}\text { Sáez-Francàs, } \\
2014\end{array}$} & \multirow{2}{*}{ IGT } & PD & 56 & 62.64 & 9.06 & 32.14 & 4.31 & 3.65 & 18.41 & 5.82 & MMSE & . & . & HAM-D & 3.64 & 3.37 \\
\hline & & Fatigue & 33 & 61.73 & 9.85 & 39.4 & 4.94 & 3.44 & 20.85 & 6.31 & & Excluded if $<26$ & . & & 8.64 & 7.06 \\
\hline \multirow{2}{*}{ Timmer, 2017} & \multirow{2}{*}{$\begin{array}{l}\text { Rewarded task- } \\
\text { switching paradigm }\end{array}$} & $\mathrm{PD}$ & 22 & 61.1 & 7.6 & 36.4 & & & 21.9 & 6.8 & MMSE & 28.6 & 1.2 & $\mathrm{BDI}$ & 4.3 & 2.3 \\
\hline & & MDD & 19 & 58.4 & 5.3 & 36.8 & & & & & & 28.5 & 1.3 & & 8.7 & 5 \\
\hline
\end{tabular}

PD = Parkinson's disease, HC = healthy controls, IGT = lowa Gambling Task, ICD = Impulse control disorder, MDD = Major depressive disorder, SD = standard deviation, IQR = interquartile range, ' '.' = not reported, '-' = not applicable, UPDRS = Unified Parkinson's Disease Rating Scale, MMSE = Mini-mental state examination, MoCA = Montreal cognitive assessment, MDRS = Mattis dementia rating scale, BDI = Beck depression inventory, HAM-D = Hamilton

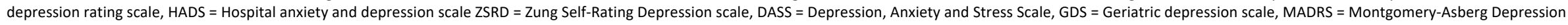
Rating Scale, SDS = Self-rated depression scale

Supplement table 3. Response Vigor and Reward Bias study characteristics and participant demographics.

\begin{tabular}{|c|c|c|c|c|c|c|c|c|c|c|c|c|c|c|c|c|}
\hline \multicolumn{4}{|c|}{ Response vigor in Parkinson's vs Healthy Controls } & \multicolumn{2}{|c|}{ Age } & \multirow[b]{2}{*}{$\begin{array}{c}\% \\
\text { female }\end{array}$} & \multicolumn{2}{|c|}{ Disease duration } & \multicolumn{2}{|c|}{ UPDRS } & \multicolumn{3}{|c|}{ Cognition } & \multicolumn{3}{|c|}{ Depression } \\
\hline Study & Task & Group & $\mathrm{n}$ & Mean & SD & & Mean & SD & Mean & SD & $\begin{array}{l}\text { Cognitive } \\
\text { measure }\end{array}$ & Mean & SD & $\begin{array}{c}\text { Depression } \\
\text { measure }\end{array}$ & Mean & SD \\
\hline \multirow{2}{*}{ Muhammed, 2016} & \multirow{2}{*}{$\begin{array}{l}\text { Speed of gaze } \\
\text { shifting task }\end{array}$} & PD & 16 & 67.3 & 6.4 & & 3.2 & 2.2 & 19.4 & 9.8 & MoCA & 27.8 & 2.3 & BDI & 11.7 & 5.5 \\
\hline & & $\mathrm{HC}$ & 31 & 65.9 & 5.6 & & - & - & - & - & & 28.2 & 1.6 & & 15.2 & 7.8 \\
\hline \multirow{2}{*}{ Renfroe, 2016} & \multirow{2}{*}{$\begin{array}{l}\text { Rewarded speed of } \\
\text { response to specific } \\
\text { visual stimuli }\end{array}$} & PD & 18 & 66 & 7.99 & 22.2 & 8.39 & 4.57 & 26 & 9.55 & & 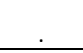 & . & $\mathrm{BDI}$ & 7.82 & 4.85 \\
\hline & & $\mathrm{HC}$ & 15 & 70 & 6.94 & 40 & - & - & - & - & & & & & 1.8 & 2.44 \\
\hline \multirow{2}{*}{ Timmer, 2018} & \multirow{2}{*}{$\begin{array}{l}\text { Stroop-like } \\
\text { incentive task } \\
\text { switching }\end{array}$} & PD & 23 & 61 & 7.4 & 39.13 & 4.5 & 2.2 & 21.8 & 6.7 & MMSE & 28.5 & 1.3 & $\mathrm{BDI}$ & 4.1 & 2.3 \\
\hline & & $\mathrm{HC}$ & 23 & 60.9 & 5.9 & 39.13 & - & - & - & - & & 28.8 & 1.2 & & 3.1 & 2.1 \\
\hline \multicolumn{4}{|c|}{ Response vigor in Parkinson's with psychiatric syndrome vs without } & \multicolumn{2}{|c|}{ Age } & & \multicolumn{2}{|c|}{ Disease duration } & \multicolumn{2}{|c|}{ UPDRS } & \multicolumn{3}{|c|}{ Cognition } & \multicolumn{3}{|c|}{ Depression } \\
\hline Study & Task & Group & $\mathrm{n}$ & Mean & SD & $\begin{array}{c}\% \\
\text { female }\end{array}$ & Mean & SD & Mean & SD & $\begin{array}{l}\text { Cognitive } \\
\text { measure }\end{array}$ & Mean & SD & $\begin{array}{c}\text { Depression } \\
\text { measure }\end{array}$ & Mean & SD \\
\hline \multirow{2}{*}{ Drew, 2020} & \multirow{2}{*}{$\begin{array}{l}\text { Speed of gaze } \\
\text { shifting task }\end{array}$} & PD & 26 & 67.19 & 5.92 & 26.9 & 4.87 & 4.09 & 18.62 & 9.38 & MoCA & 27.77 & 1.95 & $\mathrm{BDI}$ & 13 & 7.1 \\
\hline & & $I C D$ & 23 & 63.7 & 7.56 & 47.82 & 8.71 & 4.25 & 24.22 & 16.94 & & 27.39 & 2.55 & & 12.26 & 5.84 \\
\hline \multirow{2}{*}{ Evans, 2010} & \multirow{2}{*}{$\begin{array}{c}\text { Card arranging } \\
\text { reward responsivity } \\
\text { test }\end{array}$} & $\mathrm{PD}$ & 20 & 59.5 & 7.9 & & 13.6 & 8 & 19.2 & 2.5 & MMSE & 29.1 & $(27-30)$ & GDS & 9 & 4.9 \\
\hline & & DDS & 20 & 55.4 & 7.6 & & 14 & 5.7 & 22.7 & 2.5 & & 29 & $(24-30)$ & & 17.6 & 6.4 \\
\hline \multirow{2}{*}{ Lawrence, 2011} & \multirow{2}{*}{ Spatial search task } & PD & 10 & 59.6 & 6.4 & & & . & 25.6 & 11 & MMPD & 25.6 & 11 & GDS & 4.7 & 2 \\
\hline & & Apathy & 10 & 61.7 & 6.1 & & & . & 28.2 & 11.4 & & 29.3 & 2.8 & & 8.2 & 3.5 \\
\hline \multirow{2}{*}{ Muhammed, 2016} & \multirow{2}{*}{$\begin{array}{l}\text { Speed of gaze } \\
\text { shifting task }\end{array}$} & $\mathrm{PD}$ & 16 & 65.9 & 5.6 & 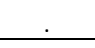 & 6.8 & 4.6 & 18.4 & 2.6 & MoCA & 28.2 & 1.6 & $\mathrm{BDI}$ & 11.7 & 5.5 \\
\hline & & Apathy & 14 & 67.3 & 6.4 & & 3.2 & 2.2 & 20.4 & 2.4 & & 27.8 & 2.3 & & 15.2 & 7.8 \\
\hline \multirow{2}{*}{ Timmer, 2018} & \multirow{2}{*}{$\begin{array}{l}\text { Stroop-like } \\
\text { incentive task } \\
\text { switching }\end{array}$} & $\mathrm{PD}$ & 23 & 61 & 7.4 & 39.13 & 4.5 & 2.2 & 21.8 & 6.7 & MMSE & 28.5 & 1.3 & $\mathrm{BDI}$ & 4.1 & 2.3 \\
\hline & & Depression & 22 & 58.4 & 5.7 & 36.36 & 5 & 3.5 & 23.1 & 9.6 & & 28.4 & 1.4 & & 9.6 & 6.1 \\
\hline
\end{tabular}

mental state examination, MoCA = Montreal cognitive assessment, MMPD $=$ Mini-mental Parkinson's examination BDI = Beck depression inventory, GDS $=$ Geriatric depression scale 
Supplement table 4. Study summary statistics, tasks and measures included in the Meta-Analysis of Parkinson's versus healthy controls.

\begin{tabular}{|c|c|c|c|c|c|c|c|c|c|c|c|c|c|c|}
\hline \multicolumn{13}{|c|}{ Reward processing summary statistics for Parkinson's disease patients versus healthy controls } & \multicolumn{2}{|c|}{ Tasks and Measures Included in the Meta-Analysis. } \\
\hline Author, Year & $\begin{array}{l}\text { Categ } \\
\text { ory }\end{array}$ & OFF/ON & $\begin{array}{l}\mathrm{N} \\
(\mathrm{HC}) \\
\end{array}$ & $\begin{array}{ll}N \\
(\mathrm{PD}) \\
\end{array}$ & $\mathrm{t}$ & $\mathrm{F}$ & $\begin{array}{l}\mathrm{M} \\
(\mathrm{HC}) \\
\end{array}$ & $\begin{array}{l}\mathrm{SD} \\
(\mathrm{HC})\end{array}$ & $\begin{array}{l}\mathrm{M} \\
(\mathrm{PD}) \\
\end{array}$ & \begin{tabular}{|l|}
$\mathrm{SD}$ \\
$(\mathrm{PD})$ \\
\end{tabular} & $d$ & Vard & Task & Measure \\
\hline Bayard et al, 2016 (ON) & ov & ON & 96 & 78 & & & 6.90 & 7.65 & 2.77 & 8.41 & 0.52 & 0.02 & Game of dice task & Net score \\
\hline Chong et al, 2015 (ON) & ov & ON & 26 & 26 & & & & & & & 0.35 & 0.08 & $\begin{array}{l}\text { Effort based decision making task } \\
\text { (apple gathering) }\end{array}$ & $\begin{array}{l}\text { Effort indifference point (mean effect size across } \\
\text { stake levels, fig 4) }\end{array}$ \\
\hline Chong et al, 2015 (OFF) & ov & OFF & 26 & 26 & & $\begin{array}{r}2.7 \\
0\end{array}$ & & & & & 0.46 & 0.08 & $\begin{array}{l}\text { Effort based decision making task } \\
\text { (apple gathering) }\end{array}$ & Effort indifference point \\
\hline Brandt et al, 2015 (ON) & ov & ON & 15 & 15 & & & $\begin{array}{r}14.9 \\
3 \\
\end{array}$ & $\begin{array}{r}15.7 \\
2\end{array}$ & $\begin{array}{r}- \\
8.67 \\
\end{array}$ & $\begin{array}{r}17.6 \\
7 \\
\end{array}$ & 1.41 & 0.17 & Game of dice task & Net score (fig 1) \\
\hline $\begin{array}{l}\text { Kobayashi et al, } 2019 \\
\text { (ON) }\end{array}$ & ov & ON & 21 & 15 & & & 0.21 & 0.78 & 0.30 & 0.66 & 0.12 & 0.11 & Economic choice task & $\begin{array}{l}\text { Relative risk aversion coefficient (fig 2C, first } \\
\text { session) }\end{array}$ \\
\hline $\begin{array}{l}\text { Kobayashi et al, } 2019 \\
\text { (OFF) }\end{array}$ & ov & OFF & 21 & 15 & & & 0.21 & 0.78 & 0.97 & 0.70 & 1.02 & 0.13 & Economic choice task & $\begin{array}{l}\text { Relative risk aversion coefficient (fig 2C, first } \\
\text { session) }\end{array}$ \\
\hline $\begin{array}{l}\text { Le Heron et al, } 2018 \\
\text { (ON) }\end{array}$ & ov & ON & 32 & 18 & $\begin{array}{r}1.3 \\
0\end{array}$ & & & & & & 0.38 & 0.09 & $\begin{array}{l}\text { Effort based decision making task } \\
\text { (apple gathering) }\end{array}$ & Mean difference in proportion of offers accepted \\
\hline $\begin{array}{l}\text { Mcguigan et al, } 2018 \\
\text { (ON) }\end{array}$ & ov & ON & 20 & 20 & $\begin{array}{r}- \\
0.3 \\
2\end{array}$ & & & & & & 0.10 & 0.10 & Cognitive effort task & Mean difference in k-value \\
\hline $\begin{array}{l}\text { Mcguigan et al, } 2018 \\
\text { (OFF) }\end{array}$ & ov & OFF & 20 & 20 & $\begin{array}{r}2.7 \\
9\end{array}$ & & & & & & 0.88 & 0.11 & Cognitive effort task & Mean difference in k-value \\
\hline Sharp et al, 2013 (ON) & ov & ON & 18 & 18 & $\begin{array}{l}0.4 \\
3\end{array}$ & & & & & & 0.14 & 0.07 & Vancouver gambling task & Gain phase adjust y intercept \\
\hline Sharp et al, 2013 (OFF) & ov & OFF & 18 & 18 & $\begin{array}{l}1.7 \\
9 \\
\end{array}$ & & & & & & 0.60 & 0.07 & Vancouver gambling task & Gain phase adjust y intercept \\
\hline Torta et al, 2009 (ON) & ov & ON & 13 & 15 & & & & & & & 0.24 & 0.14 & Cambridge gamble task & Average bet (across risk levels) \\
\hline Torta et al, 2009 (OFF) & ov & OFF & 13 & 15 & & & & & & & 0.23 & 0.14 & Cambridge gamble task & Average bet (across risk levels) \\
\hline Cools et al, 2003 (ON) & ov & ON & 12 & 12 & & & $\begin{array}{l}54.9 \\
1\end{array}$ & $\begin{array}{l}12.0 \\
9\end{array}$ & $\begin{array}{l}59.6 \\
5 \\
\end{array}$ & $\begin{array}{ll}16.1 \\
1\end{array}$ & 0.33 & 0.17 & Incentivised decision making task & $\begin{array}{l}\text { Mean \% bets across ascending \& descending } \\
\text { conditions }\end{array}$ \\
\hline Cools et al, 2003 (OFF) & ov & OFF & 12 & 12 & & & $\begin{array}{l}54.9 \\
1\end{array}$ & $\begin{array}{l}12.0 \\
9\end{array}$ & $\begin{array}{ll}58.9 \\
5\end{array}$ & $\begin{array}{l}15.7 \\
3\end{array}$ & 0.29 & 0.17 & Incentivised decision making task & $\begin{array}{l}\text { Mean \% bets across ascending \& descending } \\
\text { conditions }\end{array}$ \\
\hline Le Bouc et al, 2016 (ON) & ov & ON & 25 & 24 & & & $\begin{array}{r}80.8 \\
9 \\
\end{array}$ & 7.75 & $\begin{array}{r}78.5 \\
6 \\
\end{array}$ & $\begin{array}{r}12.4 \\
9 \\
\end{array}$ & 0.23 & 0.08 & $\begin{array}{l}\text { Effort based decision making } \\
\text { task: Binary choice task }\end{array}$ & $\begin{array}{l}\begin{array}{l}\text { Choice of effort level at the highest stake level. } \\
\text { (fig 4D) }\end{array} \\
\end{array}$ \\
\hline $\begin{array}{l}\text { Le Bouc et al, } 2016 \\
\text { (OFF) }\end{array}$ & ov & OFF & 25 & 24 & $\begin{array}{r}3.5 \\
1 \\
\end{array}$ & & & & & & 1.00 & 0.09 & $\begin{array}{l}\text { Effort based decision making } \\
\text { task: Binary choice task }\end{array}$ & $\begin{array}{l}\text { Choice of effort level at the highest stake level. } \\
\text { (fig 4D) }\end{array}$ \\
\hline $\begin{array}{l}\text { Castrioto et al, } 2015 \\
\text { (ON) }\end{array}$ & RL & ON & 24 & 20 & & & 4.38 & $\begin{array}{r}40.2 \\
2 \\
\end{array}$ & $\begin{array}{r}- \\
1.79 \\
\end{array}$ & $\begin{array}{r}44.7 \\
2 \\
\end{array}$ & 0.15 & 0.09 & lowa Gambling Task & Baseline final round mean score. (fig $1 \mathrm{~A})$ \\
\hline $\begin{array}{l}\text { lastrioto et al, } 2015 \\
\text { (OFF) }\end{array}$ & RL & OFF & 24 & 20 & & & 4.38 & $\begin{array}{r}40.2 \\
2 \\
\end{array}$ & 0.98 & $\begin{array}{r}40.3 \\
4 \\
\end{array}$ & 0.08 & 0.09 & lowa Gambling Task & Baseline final round mean score. (fig $1 \mathrm{~A})$ \\
\hline $\begin{array}{l}\text { Balusubramani et al, } \\
2015 \text { (ON) }\end{array}$ & $\mathrm{RL}$ & ON & 20 & 14 & & & $\begin{array}{r}64.1 \\
0\end{array}$ & $\begin{array}{r}84.8 \\
4\end{array}$ & $\begin{array}{r}61.5 \\
4\end{array}$ & $\begin{array}{r}78.6 \\
9\end{array}$ & 0.03 & 0.12 & $\begin{array}{l}\text { Probabilistic rewarded } \\
\text { categorisation learning task }\end{array}$ & \% Optimality during expected reward (fig 3A) \\
\hline $\begin{array}{l}\text { Balusubramani et al, } \\
2015 \text { (OFF) }\end{array}$ & RL & OFF & 20 & 26 & & & $\begin{array}{r}64.1 \\
0\end{array}$ & $\begin{array}{r}84.8 \\
4\end{array}$ & $\begin{array}{r}43.0 \\
7 \\
\end{array}$ & $\begin{array}{r}99.3 \\
8 \\
\end{array}$ & 0.23 & 0.09 & $\begin{array}{l}\text { Probabilistic rewarded } \\
\text { categorisation learning task }\end{array}$ & \% Optimality during expected reward (fig 3A) \\
\hline Bodi et al, 2009 (ON) & RL & Medicated & 20 & 22 & & & $\begin{array}{r}81.1 \\
8\end{array}$ & $\begin{array}{r}18.4 \\
3 \\
\end{array}$ & $\begin{array}{r}89.1 \\
8 \\
\end{array}$ & $\begin{array}{r}17.1 \\
2 \\
\end{array}$ & $\begin{array}{r}- \\
0.45 \\
\end{array}$ & 0.10 & $\begin{array}{l}\text { Feedback-based probabilistic } \\
\text { classification task }\end{array}$ & Final block \% optimal decisions (fig 2) \\
\hline $\begin{array}{l}\text { Bodi et al, } 2009 \text { (Never } \\
\text { medicated) }\end{array}$ & $\mathrm{RL}$ & $\begin{array}{l}\text { Never } \\
\text { medicated }\end{array}$ & 20 & 26 & & & $\begin{array}{r}81.1 \\
8 \\
\end{array}$ & $\begin{array}{r}18.4 \\
3 \\
\end{array}$ & $\begin{array}{r}59.5 \\
3 \\
\end{array}$ & $\begin{array}{r}18.6 \\
1 \\
\end{array}$ & 1.17 & 0.10 & $\begin{array}{l}\text { Feedback-based probabilistic } \\
\text { classification task }\end{array}$ & Final block \% optimal decisions (fig 2) \\
\hline Buelow et al, 2014 (ON) & RL & ON & 14 & 24 & & & & & & & 1.38 & 0.14 & lowa Gambling Task & Final block score (authors provided) \\
\hline
\end{tabular}




\begin{tabular}{|c|c|c|c|c|c|c|c|c|c|c|c|c|c|c|}
\hline Piray et al, 2014 (ON) & $\mathrm{RL}$ & ON & 20 & 15 & & & 0.12 & 0.16 & 0.14 & 0.20 & $0.10^{-}$ & 0.12 & Probabilistic learning task & Actor's learning rate (fig 6C) \\
\hline Piray et al, 2014 (OFF) & $\mathrm{RL}$ & OFF & 20 & 25 & & & 0.12 & 0.16 & 0.08 & 0.13 & 0.29 & 0.09 & Probabilistic learning task & Actor's learning rate (fig 6C) \\
\hline Yildirim et al, 2020 (ON) & $\mathrm{RL}$ & ON & 37 & 39 & & & 0.65 & 5.72 & 0.11 & 5.77 & 0.13 & 0.05 & lowa Gambling Task & Final block mean score (author provided) \\
\hline $\begin{array}{l}\text { Gescheidt et al, } 2012 \\
\text { (ON) }\end{array}$ & RL & ON & 20 & 19 & & & $\begin{array}{r}10.3 \\
0\end{array}$ & $\begin{array}{r}29.4 \\
2\end{array}$ & 6.00 & $\begin{array}{r}25.2 \\
6 \\
\end{array}$ & 0.59 & 0.11 & lowa Gambling Task & Mean total score \\
\hline $\begin{array}{l}\text { Herzallah et al, } 2017 \\
\text { (ON) }\end{array}$ & $\mathrm{RL}$ & ON & 15 & 17 & & & $\begin{array}{r}76.4 \\
9\end{array}$ & $\begin{array}{r}17.3 \\
6\end{array}$ & $\begin{array}{r}72.1 \\
0\end{array}$ & $\begin{array}{r}16.6 \\
2\end{array}$ & 0.26 & 0.13 & $\begin{array}{l}\text { Feedback-based probabilistic } \\
\text { classification task }\end{array}$ & $\begin{array}{l}\text { Mean \% optimal responses for positive feedback } \\
\text { (fig 5A) }\end{array}$ \\
\hline $\begin{array}{l}\text { lousden et al, } 2010 \\
\text { (ON) }\end{array}$ & $\mathrm{RL}$ & ON & 20 & 18 & & & $\begin{array}{r}56.5 \\
0 \\
\end{array}$ & $\begin{array}{r}22.4 \\
0 \\
\end{array}$ & $\begin{array}{r}27.0 \\
0 \\
\end{array}$ & $\begin{array}{r}19.1 \\
0 \\
\end{array}$ & 1.41 & 0.13 & Salience Attribution Test & $\begin{array}{l}\text { Visual analogue scale rating for high probability } \\
\text { stimuli }\end{array}$ \\
\hline Mapelli et al, 2014 (ON) & RL & ON & 15 & 15 & & & 9.81 & $\begin{array}{r}10.1 \\
9\end{array}$ & 4.74 & 5.11 & 0.63 & 0.14 & lowa Gambling Task & Final block mean score (fig 1 ) \\
\hline Mimura et al, 2006 (ON) & $\mathrm{RL}$ & ON & 20 & 18 & & & 3.70 & 7.35 & 0.33 & 6.59 & 0.58 & 0.11 & lowa Gambling Task & Final 50 cards mean score \\
\hline $\begin{array}{l}\text { Pagonabarraga et al, } \\
2007 \text { (ON) }\end{array}$ & $\mathrm{RL}$ & ON & 31 & 35 & & & 6.10 & $\begin{array}{r}17.0 \\
0\end{array}$ & $\begin{array}{r}10 . \overline{8} \\
0\end{array}$ & $\begin{array}{r}19.0 \\
0\end{array}$ & 0.93 & 0.07 & lowa Gambling Task & Mean total score \\
\hline $\begin{array}{l}\text { Paz-Alonso et al, } 2019 \\
\text { (ON) }\end{array}$ & RL & ON & 18 & 17 & & & $\begin{array}{r}40.6 \\
0\end{array}$ & $\begin{array}{r}18.8 \\
2 \\
\end{array}$ & $\begin{array}{r}41.8 \\
0\end{array}$ & $\begin{array}{r}15.3 \\
8 \\
\end{array}$ & 0.07 & 0.11 & lowa Gambling Task & $\begin{array}{l}\text { Mean difference \% optimal choice, final block. } \\
\text { (supplement fig 1) }\end{array}$ \\
\hline $\begin{array}{l}\text { Garofalo et al, } 2017 \\
\text { (ON) }\end{array}$ & $\mathrm{RL}$ & ON & 24 & 17 & & & $\begin{array}{r}21.5 \\
0\end{array}$ & 5.26 & $\begin{array}{r}15.1 \\
5\end{array}$ & 6.68 & 1.08 & 0.11 & Instrumental conditioning task & Reward learning index (fig 2B) \\
\hline $\begin{array}{l}\text { Czernecki et al, } 2002 \\
\text { (ON) }\end{array}$ & RL & ON & 28 & 23 & & & 8.32 & $\begin{array}{r}11.0 \\
6\end{array}$ & 2.86 & $\begin{array}{r}16.5 \\
9 \\
\end{array}$ & 0.40 & 0.08 & lowa Gambling Task & Final block mean score (fig 1 , second session) \\
\hline $\begin{array}{l}\text { Czernecki et al, } 2002 \\
\text { (OFF) }\end{array}$ & RL & OFF & 28 & 23 & & & 8.32 & $\begin{array}{r}11.0 \\
6 \\
\end{array}$ & 5.36 & $\begin{array}{r}10.8 \\
9 \\
\end{array}$ & 0.27 & 0.08 & Iowa Gambling Task & Final block mean score (fig 1 , second session) \\
\hline Graef et al, 2010 (ON) & RL & ON & 15 & 14 & $\begin{array}{r}1.8 \\
0\end{array}$ & & $\begin{array}{r}68.4 \\
9\end{array}$ & $\begin{array}{r}14.0 \\
7\end{array}$ & $\begin{array}{r}60.0 \\
7 \\
\end{array}$ & $\begin{array}{r}11.0 \\
8 \\
\end{array}$ & 0.67 & 0.15 & Instrumental learning task & $\begin{array}{l}\text { \% correct choices with constant reward } \\
\text { contingencies }\end{array}$ \\
\hline Graef et al, 2010 (OFF) & RL & OFF & 15 & 14 & $\begin{array}{r}2.1 \\
9 \\
\end{array}$ & & $\begin{array}{r}68.4 \\
9 \\
\end{array}$ & $\begin{array}{r}14.0 \\
7 \\
\end{array}$ & $\begin{array}{r}59.0 \\
5 \\
\end{array}$ & 8.86 & 0.81 & 0.15 & Instrumental learning task & $\begin{array}{l}\text { \% correct choices with constant reward } \\
\text { contingencies }\end{array}$ \\
\hline Thiel et al, 2003 (OFF) & $\mathrm{RL}$ & OFF & 5 & 5 & & & 53.6 & 15.8 & 61.2 & 17.1 & 0.46 & 0.41 & lowa Gambling Task & Mean number of advantageous cards selected. \\
\hline $\begin{array}{l}\text { Perretta et al, } 2005 \\
\text { (late PD) }\end{array}$ & RL & ON late PD & 19 & 16 & & & 7.80 & 1.31 & 6.80 & 1.60 & 0.69 & 0.12 & lowa Gambling Task & Mean total score \\
\hline $\begin{array}{l}\text { Perretta et al, } 2005 \\
\text { (early PD) }\end{array}$ & $\mathrm{RL}$ & ON early PD & 19 & 16 & & & 7.80 & 1.31 & 7.60 & 1.60 & 0.14 & 0.12 & lowa Gambling Task & Mean total score \\
\hline $\begin{array}{l}\text { Kobayakawa et al, } 2008 \\
\text { (ON) }\end{array}$ & $\mathrm{RL}$ & ON & 22 & 34 & & $\begin{array}{r}4.8 \\
0\end{array}$ & 4.90 & $\begin{array}{r}12.2 \\
0\end{array}$ & $\begin{array}{r}16.0 \\
0\end{array}$ & $\begin{array}{r}21.5 \\
7\end{array}$ & 0.60 & 0.08 & lowa Gambling Task & $\begin{array}{l}\text { Group difference in choice patterns } \\
\text { (advantageous - disadvantageous) }\end{array}$ \\
\hline $\begin{array}{l}\text { Kobayakawa et al, } 2010 \\
\text { (ON) }\end{array}$ & RL & ON & 22 & 14 & & $\begin{array}{r}2.9 \\
6 \\
\end{array}$ & & & & & 0.59 & 0.12 & lowa Gambling Task & $\begin{array}{l}\text { Group difference in choice patterns } \\
\text { (advantageous - disadvantageous) }\end{array}$ \\
\hline Poletti et al, 2010 (OFF) & $\mathrm{RL}$ & $\begin{array}{l}\text { OFF (de } \\
\text { novo) }\end{array}$ & 25 & 30 & & & 6.00 & 6.82 & 3.07 & $\begin{array}{r}12.0 \\
7\end{array}$ & 0.29 & 0.07 & lowa Gambling Task & Mean total score \\
\hline Delazer et al, 2009 (ON) & $\mathrm{RL}$ & ON & 20 & 20 & & $\begin{array}{r}8.8 \\
1\end{array}$ & & & & & 0.94 & 0.11 & lowa Gambling Task & $\begin{array}{l}\text { Group difference in choice patterns } \\
\text { (advantageous - disadvantageous) }\end{array}$ \\
\hline $\begin{array}{l}\text { Euteneuer et al, } 2009 \\
\text { (ON) }\end{array}$ & RL & ON & 23 & 21 & & $\begin{array}{r}0.6 \\
5 \\
\end{array}$ & & & & & 0.24 & 0.09 & lowa Gambling Task & $\begin{array}{l}\text { Group difference in choice patterns } \\
\text { (advantageous - disadvantageous) }\end{array}$ \\
\hline $\begin{array}{l}\text { Ibarretxe-Bilbao et al, } \\
2009(\mathrm{ON})\end{array}$ & $\mathrm{RL}$ & ON & 24 & 24 & & $\begin{array}{r}14 . \\
56 \\
\end{array}$ & & & & & 1.10 & 0.10 & Iowa Gambling Task & $\begin{array}{l}\text { Group difference in choice patterns } \\
\text { (advantageous - disadvantageous) }\end{array}$ \\
\hline Pignatti et al, 2012 (ON) & RL & ON & 16 & 15 & & & $\begin{array}{r}12.5 \\
0 \\
\end{array}$ & $\begin{array}{r}14.8 \\
7 \\
\end{array}$ & 7.47 & $\begin{array}{r}16.0 \\
6 \\
\end{array}$ & 0.33 & 0.13 & lowa Gambling Task & Total score over the last 50 choices \\
\hline Evens et al, 2015 (ON) & $\mathrm{RL}$ & ON & 32 & 32 & & & 7.31 & $\begin{array}{r}20.7 \\
8\end{array}$ & 2.09 & $\begin{array}{r}16.2 \\
7\end{array}$ & 0.28 & 0.06 & lowa Gambling Task & Mean total score \\
\hline
\end{tabular}




\begin{tabular}{|c|c|c|c|c|c|c|c|c|c|c|c|c|c|c|}
\hline Xi et al, 2015 (ON) & RL & ON & 15 & 15 & & \begin{tabular}{r|}
10. \\
67 \\
\end{tabular} & & & & & 1.19 & 0.16 & lowa Gambling Task & $\begin{array}{l}\text { Group difference in choice patterns } \\
\text { (advantageous - disadvantageous) }\end{array}$ \\
\hline $\begin{array}{l}\text { Cavanagh et al, } 2017 \\
\text { (ON) }\end{array}$ & RL & ON & 28 & 28 & $\begin{array}{r}1.6 \\
4 \\
\end{array}$ & & & & & & 0.44 & 0.07 & Cost of conflict task & $\begin{array}{l}\% \text { selection of most (A) vs least }(D) \text { rewarding } \\
\text { stimuli }\end{array}$ \\
\hline $\begin{array}{l}\text { Cavanagh et al, } 2017 \\
\text { (OFF) }\end{array}$ & RL & OFF & 28 & 28 & $\begin{array}{r}1.4 \\
4\end{array}$ & & & & & & 0.38 & 0.07 & Cost of conflict task & $\begin{array}{l}\text { \% selection of most (A) vs least (D) rewarding } \\
\text { stimuli }\end{array}$ \\
\hline $\begin{array}{l}\text { Muhammed et al, } 2016 \\
\text { (ON) }\end{array}$ & RV & ON & 31 & 30 & $\begin{array}{r}5.5 \\
0 \\
\end{array}$ & & & & & & 0.60 & 0.07 & $\begin{array}{l}\text { Rewarded speed of gaze shifting } \\
\text { task }\end{array}$ & Reward sensitivity in peak velocity \\
\hline Timmer et al, 2018 (ON) & RV & ON & 23 & 23 & & & 5.00 & 4.50 & $\begin{array}{l}14.4 \\
0\end{array}$ & 4.40 & 2.11 & 0.14 & $\begin{array}{l}\text { Rewarded task switching } \\
\text { paradigm }\end{array}$ & Reward related speeding 'repeat' condition \\
\hline $\begin{array}{l}\text { Timmer et al, } 2018 \\
\text { (OFF) }\end{array}$ & RV & OFF & 23 & 23 & & & 5.00 & 4.50 & 3.80 & 4.70 & 0.26 & 0.09 & $\begin{array}{l}\text { Rewarded task switching } \\
\text { paradigm }\end{array}$ & Reward related speeding 'repeat' condition \\
\hline
\end{tabular}

Supplement table 5. Study summary statistics, tasks and measures included in the Meta-Analysis of Parkinson's with \& without psychiatric syndrome

\begin{tabular}{|c|c|c|c|c|c|c|c|c|c|c|c|c|c|c|}
\hline \multicolumn{13}{|c|}{ Reward processing summary statistics for Parkinson's disease versus Parkinson's disease plus psychiatric syndrome } & \multicolumn{2}{|c|}{ Tasks and Measures Included in the Meta-Analysis. } \\
\hline Author, Year & Category & $\begin{array}{l}\text { Psychiatric } \\
\text { syndrome }\end{array}$ & $N(P D)$ & $\begin{array}{l}\mathrm{N} \\
(\mathrm{PD}+\mathrm{PSY} \\
\mathrm{CH})\end{array}$ & $\mathrm{t}$ & $\mathrm{F}$ & $\begin{array}{l}M \\
(P D)\end{array}$ & $\begin{array}{l}\text { SD } \\
(P D)\end{array}$ & $\begin{array}{l}M(P D \\
+ \text { Psych) }\end{array}$ & $\begin{array}{l}\text { SD } \\
\text { (PD+Psyc } \\
\text { h) }\end{array}$ & d & Vard & Task & Measure \\
\hline $\begin{array}{l}\text { Balusubramani } \\
\text { et al, } 2015 \text { (ON) }\end{array}$ & RL & ICD & 14 & 16 & & & $\begin{array}{r}61.5 \\
4 \\
\end{array}$ & $\begin{array}{r}78.6 \\
9\end{array}$ & 78.97 & 61.56 & -0.25 & 0.13 & $\begin{array}{l}\text { Probabilistic reward and } \\
\text { punishment learning task }\end{array}$ & $\begin{array}{l}\text { \% Optimality during expected reward (fig } \\
3 A \text { ) }\end{array}$ \\
\hline $\begin{array}{l}\text { Piray et al, } \\
2014 \text { (ON) } \\
\end{array}$ & RL & ICD & 15 & 16 & & & $\begin{array}{r}0.13 \\
5 \\
\end{array}$ & $\begin{array}{r}0.19 \\
8 \\
\end{array}$ & 0.067 & 0.06 & 0.47 & 0.13 & Probabilistic learning task & Actor's learning rate (fig 6C) \\
\hline $\begin{array}{l}\text { Housden et al, } \\
2010 \text { (ON) }\end{array}$ & $\mathrm{RL}$ & ICD & 18 & 18 & & & 27 & 19.1 & 42.7 & 19.7 & -0.81 & 0.12 & Salience Attribution Test & $\begin{array}{l}\text { Visual analogue scale rating for high } \\
\text { probability stimuli }\end{array}$ \\
\hline $\begin{array}{l}\text { Biars et al, } 2019 \\
\text { (ON) }\end{array}$ & RL & ICD & 24 & 24 & & & 3.5 & 9.7 & 2.75 & 8.2 & 0.08 & 0.08 & lowa Gambling Task & Final block score (authors provided) \\
\hline $\begin{array}{l}\text { Balconi et al, } \\
2018 \text { (ON) }\end{array}$ & $\mathrm{RL}$ & PG & 20 & 17 & & $\begin{array}{r}7 . \\
60 \\
\end{array}$ & & & & & 0.91 & 0.12 & lowa Gambling Task & $\begin{array}{l}\text { Group difference in choice patterns } \\
\text { (advantageous - disadvantageous) }\end{array}$ \\
\hline $\begin{array}{l}\text { Paz-Alonso et } \\
\text { al, } 2019 \text { (ON) }\end{array}$ & RL & ICD & 17 & 18 & & & $\begin{array}{r}41.8 \\
0\end{array}$ & $\begin{array}{r}15.3 \\
8\end{array}$ & 27.00 & 13.94 & 1.01 & 0.13 & lowa Gambling Task & $\begin{array}{l}\text { Mean difference \% optimal choice, final } \\
\text { block. (supplement fig 1) }\end{array}$ \\
\hline $\begin{array}{l}\text { Pineau et al, } \\
2016 \text { (ON) }\end{array}$ & $\mathrm{RL}$ & ICD & 20 & 17 & & & $\begin{array}{r}15.0 \\
0\end{array}$ & $\begin{array}{r}15.5 \\
6\end{array}$ & 14.00 & 9.63 & 0.08 & 0.11 & lowa Gambling Task & $\begin{array}{l}\text { Mean number of cards selected from } \\
\text { winning deck }\end{array}$ \\
\hline $\begin{array}{l}\text { Bentivoglio et } \\
\text { al, } 2012 \text { (ON) }\end{array}$ & $\mathrm{RL}$ & ICD & 17 & 17 & & & 8.40 & $\begin{array}{r}22.1 \\
0 \\
\end{array}$ & -4.60 & 33.10 & 0.46 & 0.12 & Iowa Gambling Task & Mean total score \\
\hline $\begin{array}{l}\text { Rossi et al, } 2010 \\
\text { (ON) }\end{array}$ & $\mathrm{RL}$ & PG & 13 & 7 & $\begin{array}{r}2 . \\
60 \\
\end{array}$ & & & & & & 1.22 & 0.26 & Iowa Gambling Task & Final block mean net score \\
\hline $\begin{array}{l}\text { Garofalo et al, } \\
2017 \text { (ON) }\end{array}$ & $\mathrm{RL}$ & Psychosis & 17 & 12 & & & $\begin{array}{r}15.1 \\
5 \\
\end{array}$ & 6.68 & 8.67 & 8.34 & 0.88 & 0.16 & Instrumental conditioning task & Reward learning index (fig 2B) \\
\hline $\begin{array}{l}\text { Sáez-Francàs et } \\
\text { al, } 2014 \text { (ON) }\end{array}$ & RL & Fatigue & 56 & 33 & & & 1.79 & $\begin{array}{r}14.8 \\
0 \\
\end{array}$ & -4.18 & 11.13 & 0.44 & 0.05 & Iowa Gambling Task & Final three blocks mean net score \\
\hline $\begin{array}{l}\text { Martinez-Horta } \\
\text { et al, } 2013 \text { (ON) }\end{array}$ & $\mathrm{RL}$ & Apathy & 17 & 20 & & & 1.82 & 5.80 & 4.10 & 4.80 & -1.12 & 0.13 & lowa Gambling Task & Final block score \\
\hline $\begin{array}{l}\text { Buelow et al, } \\
2014 \text { (ON) }\end{array}$ & $\mathrm{RL}$ & Apathy & 14 & 10 & & & $\begin{array}{r}22.1 \\
2 \\
\end{array}$ & $\begin{array}{r}16.8 \\
0 \\
\end{array}$ & -24.00 & 14.87 & 2.88 & 0.34 & lowa Gambling Task & Final block score (authors provided) \\
\hline $\begin{array}{l}\text { Herzallah et al, } \\
2017 \text { (ON) }\end{array}$ & $\mathrm{RL}$ & MDD & 17 & 13 & & & $\begin{array}{r}72.1 \\
0\end{array}$ & $\begin{array}{r}16.6 \\
2\end{array}$ & 49.30 & 22.14 & 1.19 & 0.16 & $\begin{array}{l}\text { Feedback-based probabilistic } \\
\text { classification task }\end{array}$ & $\begin{array}{l}\text { Mean \% optimal responses for positive } \\
\text { feedback (fig } 5 \mathrm{~A} \text { ) }\end{array}$ \\
\hline $\begin{array}{l}\text { Timmer et al, } \\
2017 \text { (ON) }\end{array}$ & $\mathrm{RL}$ & MDD & 22 & 19 & & & 0.10 & 0.06 & 0.07 & 0.06 & -0.50 & 0.10 & $\begin{array}{l}\text { Deterministic reversal learning } \\
\text { paradigm }\end{array}$ & Error rate for expected reward \\
\hline
\end{tabular}




\begin{tabular}{|c|c|c|c|c|c|c|c|c|c|c|c|c|c|c|}
\hline $\begin{array}{l}\text { Poletti et al, } \\
2011 \text { (OFF) }\end{array}$ & RL & Alexithymia & 12 & 12 & & & 4.17 & 8.37 & 3.00 & 6.35 & 0.16 & 0.17 & lowa Gambling Task & Final block score \\
\hline $\begin{array}{l}\text { Kobayashi et al, } \\
2019 \text { (ON) }\end{array}$ & ov & ICD & 15 & 10 & & & 0.30 & 0.66 & -0.19 & 0.17 & -0.93 & 0.18 & Economic choice task & $\begin{array}{l}\text { Relative risk aversion coefficient (fig } 2 \mathrm{C} \text {, } \\
\text { first session) }\end{array}$ \\
\hline $\begin{array}{l}\text { Le Heron et al, } \\
2018 \text { (ON) }\end{array}$ & ov & Apathy & 18 & 21 & $\begin{array}{r}2 . \\
33\end{array}$ & & & & & & 0.75 & 0.11 & $\begin{array}{l}\text { Effort based decision making } \\
\text { task (apple gathering) }\end{array}$ & $\begin{array}{l}\text { Mean difference in proportion of offers } \\
\text { accepted }\end{array}$ \\
\hline $\begin{array}{l}\text { Voon et al, } \\
2011 \text { (ON) }\end{array}$ & ov & ICD & 14 & 14 & & & & & & & -0.68 & 0.14 & Gambling task & $\begin{array}{l}\text { Proportion of risky choices in gain phase } \\
\text { (across risk levels, fig 2A) }\end{array}$ \\
\hline $\begin{array}{l}\text { Timmer et al, } \\
2018 \text { (ON) }\end{array}$ & RV & MDD & 23 & 22 & & & $\begin{array}{r}14.4 \\
0\end{array}$ & 4.40 & 17.70 & 3.90 & -0.79 & 0.10 & $\begin{array}{l}\text { Rewarded task-switching } \\
\text { paradigm }\end{array}$ & Reaction time reward benefit (repeat) \\
\hline $\begin{array}{l}\text { Evans et al, } \\
2010 \text { (ON) }\end{array}$ & RV & DDS & 20 & 20 & & $\begin{array}{r}12 \\
.7\end{array}$ & & & & & -1.13 & 0.12 & $\begin{array}{l}\text { Card arranging reward } \\
\text { responsivity objective test }\end{array}$ & Reward responsivity \\
\hline $\begin{array}{l}\text { Drew et al, } \\
2020 \text { (ON) }\end{array}$ & RV & ICD & 26 & 23 & & $\begin{array}{r}2 . \\
78\end{array}$ & & & & & -0.48 & 0.08 & $\begin{array}{l}\text { Rewarded speed of gaze } \\
\text { shifting task }\end{array}$ & Residual velocity reward sensitivity \\
\hline $\begin{array}{l}\text { Lawrence et al, } \\
2011 \text { (ON) }\end{array}$ & RV & Apathy & 10 & 10 & & $\begin{array}{c}0 . \\
38\end{array}$ & & & & & 0.20 & 0.28 & Rewarded spatial search task & Reward related speeding \\
\hline
\end{tabular}

\section{Supplement table 6. Study summary statistics, tasks and measures included in the Meta-Analysis of Parkinson's on and off dopaminergic} medication

\begin{tabular}{|c|c|c|c|c|c|c|c|c|}
\hline \multicolumn{7}{|c|}{$\begin{array}{l}\text { Reward processing summary statistics for Parkinson's disease patients ON vs OFF } \\
\text { dopaminergic medication }\end{array}$} & \multicolumn{2}{|c|}{ Tasks and Measures Included in the Meta-Analysis. } \\
\hline Author, Year & Category & $\mathrm{N}$ & $\mathrm{t}$ & $\mathrm{F}$ & d & Vard & Task & Measure \\
\hline Czernecki et al, 2002 & $\mathrm{RL}$ & 22 & & 0.05 & 0.048 & 0.046 & lowa Gambling Task & Number of advantageous minus disadvantageous choices \\
\hline Graef et al, 2010 & RL & 14 & -0.374 & & -0.100 & 0.072 & Instrumental learning task & $\%$ correct choices with constant reward contingencies \\
\hline Cavanagh et al, 2017 & RL & 28 & & 2.87 & 0.320 & 0.0375 & Value of volition task & $\begin{array}{l}\text { \% selection of A ( } 90 \% \text { reward) vs C ( } 70 \% \text { reward) - B ( } 10 \% \text { reward) } \\
\text { vs D ( } 30 \% \text { reward) stimuli }\end{array}$ \\
\hline Bodi et al, 2009 & RL & 26 & & 13.47 & 0.720 & 0.0385 & Feedback-based probabilistic classification task & Pattern of optimal decision selection over time \\
\hline Chong et al, 2015 & ov & 26 & & \multirow{3}{*}{$\begin{array}{l}25.9 \\
7.48\end{array}$} & 0.998 & 0.058 & $\begin{array}{l}\text { Effort based decision making task (apple } \\
\text { gathering) }\end{array}$ & Effort indifference point \\
\hline Kobayashi et al, 2019 & ov & 24 & & & 0.558 & 0.048 & Economic choice task & Relative risk aversion coefficient \\
\hline Le Heron et al, 2018 & ov & 39 & 2.45 & & 0.392 & 0.028 & $\begin{array}{l}\text { Effort based decision making task (apple } \\
\text { gathering) }\end{array}$ & Mean difference in proportion of offers accepted \\
\hline Mcguigan et al, 2018 & ov & 20 & 3.05 & & 0.682 & 0.062 & Cogntiive effort task & Mean difference in k-value \\
\hline Cools et al, 2003 & ov & 12 & & 5.9 & 0.701 & 0.104 & Incentivised decision making task & $\begin{array}{l}\text { Difference score of bets places in ascending and descending } \\
\text { conditions. }\end{array}$ \\
\hline Le Bouc et al, 2016 & ov & 20 & 2.76 & & 0.617 & 0.060 & $\begin{array}{l}\text { Effort based decision making task: Binary } \\
\text { choice task }\end{array}$ & Choice of effort level at the highest stake level. \\
\hline Sharp et al, 2013 & ov & 18 & 1.17 & & 0.276 & 0.058 & Vancouver gambling task & Adjusted y intercept \\
\hline Timmer et al, 2018 & RV & 23 & & 3.031 & 0.363 & 0.046 & Rewarded task switching paradigm & Reward related speeding 'repeat' condition \\
\hline Muhammed et al, 2016 & RV & 30 & & 10.8 & 0.600 & 0.039 & Rewarded speed of gaze shifting task & Reward sensitivity in peak velocity \\
\hline Evans et al, 2010 & RV & 38 & & 0.4 & 0.103 & 0.026 & $\begin{array}{l}\text { Card arranging reward responsivity objective } \\
\text { test }\end{array}$ & Reward responsivity \\
\hline Drew, 2020 & RV & 26 & & 5.178 & 0.446 & 0.042 & Rewarded speed of gaze shifting task & Residual velocity reward sensitivity \\
\hline
\end{tabular}




\section{Modified version of the Newcastle-Ottawa Scale for Assessing the Quality of Nonrandomized Studies in Meta-Analyses, used to assess} potential sources of bias.

Potential sources of bias were assessed using a modified version of the Newcastle-Ottawa Scale for Assessing the Quality of Nonrandomized Studies in Meta-Analyses. The studies are scored on:
1. PD Definition: Is the case definition adequate?
A) Cases were defined as PD according to a validated assessment tool/criteria or by an experienced clinician
B) Cases were defined as PD according to a validated assessment tool/criteria but the method for assessing PD status was not stated.
C) Cases were described as 'clinically' but no further description was given.

\section{PD Generality: Was a General sample of cases tested? \\ A) A General sample of PD was tested. \\ B) Recruitment of PD cases was restricted to a specific sub-sample (specific age range, hospitalised only etc.)}

\section{HC Selection: Selection of Controls}

A) Controls were selected from the same population as cases

B) Controls were not selected from the same population as cases

C) No description

\section{HC Definition: Definition of Controls}

A) $\mathrm{HC}$ were defined clearly defined as having no current or past psychopathology

B) Controls were not clearly defined as having no current or past psychopathology.

\section{Comparability (Comparability of cases and controls on the basis of the design or analysis)}

1. Does the study control for Age: Yes/No/Unclear

2. Does the study control for Gender: Yes/No/Unclear

3. Does the study control for IQ: Yes/No/Unclear

4. Does the study control for Socioeconomic status: Yes/No/Unclear

5. Does the study control for PD severity: Yes/No/Unclear

6. Does the study control for medication status: Yes/No/Unclear

Key: A, B, C

$\mathrm{Y}=$ yes, $\mathrm{N}=$ no, $\mathrm{N} / \mathrm{A}=$ Not applicable (such as if no healthy control group in study) 


\section{Supplement table 7. Quality rating of included studies}

\begin{tabular}{|c|c|c|c|c|c|c|c|c|c|c|c|c|}
\hline \multicolumn{3}{|c|}{ Study quality ratings } & \multicolumn{10}{|c|}{ Modified version of the Newcastle-Ottawa Scale } \\
\hline Study & Year & Category & PD Definition & PD Generality & HC Selection & HC Definition & Age & Gender & IQ & Socioeconomic status & PD severity & PD medication \\
\hline Bayard $^{1}$ & 2016 & OV & A & A & B & A & Y & $\mathrm{Y}$ & $\mathrm{N}$ & $\mathrm{N}$ & Y & $\mathrm{Y}$ \\
\hline Brandt $^{2}$ & 2015 & OV & A & A & $\mathrm{C}$ & B & $\mathrm{Y}$ & $\mathrm{Y}$ & $\mathrm{Y}$ & $\mathrm{N}$ & $\mathrm{Y}$ & $\mathrm{Y}$ \\
\hline Chong $^{3}$ & 2015 & OV & B & A & A & A & $\mathrm{Y}$ & $\mathrm{Y}$ & $\mathrm{N}$ & $\mathrm{N}$ & $\mathrm{Y}$ & $\mathrm{Y}$ \\
\hline Cools $^{4}$ & 2003 & OV & A & A & C & B & $\mathrm{Y}$ & $\mathrm{N}$ & $\mathrm{Y}$ & $\mathrm{N}$ & $\mathrm{Y}$ & $\mathrm{Y}$ \\
\hline Haagensen $^{5}$ & 2020 & OV & $\mathrm{C}$ & A & A & $B$ & $\mathrm{Y}$ & $Y$ & $\mathrm{~N}$ & $\mathrm{~N}$ & $\mathrm{Y}$ & $\mathrm{Y}$ \\
\hline Kobayashi $^{6}$ & 2019 & OV & $A$ & A & C & $B$ & $\mathrm{Y}$ & $Y$ & $\mathrm{~N}$ & $\mathrm{~N}$ & $\mathrm{Y}$ & $Y$ \\
\hline Le Bouc ${ }^{7}$ & 2016 & OV & C & $B$ & A & B & $\mathrm{Y}$ & $Y$ & $\mathrm{~N}$ & $\mathrm{~N}$ & $\mathrm{Y}$ & $\mathrm{Y}$ \\
\hline Le Heron ${ }^{8}$ & 2018 & OV & A & A & A & A & $Y$ & $Y$ & $\mathrm{~N}$ & $\mathrm{~N}$ & $\mathrm{Y}$ & $Y$ \\
\hline Mcguigan $^{9}$ & 2018 & OV & A & A & A & A & $Y$ & $Y$ & $\mathrm{~N}$ & $\mathrm{~N}$ & $Y$ & $Y$ \\
\hline Torta $^{10}$ & 2009 & OV & A & B & c & B & $Y$ & $Y$ & $\mathrm{~N}$ & $\mathrm{~N}$ & $Y$ & $Y$ \\
\hline Sharp ${ }^{11}$ & 2013 & OV & A & A & C & B & $Y$ & $\mathrm{~N}$ & $\mathrm{~N}$ & $\mathrm{~N}$ & $\mathrm{Y}$ & $\mathrm{Y}$ \\
\hline Voon $^{12}$ & 2011 & OV & A & $\mathrm{A}$ & $n / a$ & $\mathrm{n} / \mathrm{a}$ & $\mathrm{Y}$ & $Y$ & $\mathrm{~N}$ & $\mathrm{~N}$ & $\mathrm{Y}$ & $Y$ \\
\hline Balconi ${ }^{13}$ & 2018 & $\mathrm{RL}$ & A & A & $\mathrm{n} / \mathrm{a}$ & $\mathrm{n} / \mathrm{a}$ & $Y$ & $\mathrm{~N}$ & $\mathrm{~N}$ & $\mathrm{~N}$ & $\mathrm{Y}$ & $Y$ \\
\hline Balusubramani $i^{14}$ & 2015 & $\mathrm{RL}$ & C & A & c & A & $\mathrm{N}$ & $\mathrm{N}$ & $\mathrm{N}$ & $\mathrm{N}$ & $Y$ & $Y$ \\
\hline Bentivoglio ${ }^{15}$ & 2012 & $\mathrm{RL}$ & A & $A$ & $n / a$ & $n / a$ & $\mathrm{Y}$ & $Y$ & $\mathrm{Y}$ & $\mathrm{N}$ & $\mathrm{Y}$ & $\mathrm{Y}$ \\
\hline Biars' & 2019 & $\mathrm{RL}$ & $\mathrm{C}$ & $B$ & $\mathrm{n} / \mathrm{a}$ & $\mathrm{n} / \mathrm{a}$ & $Y$ & $Y$ & $\mathrm{Y}$ & $\mathrm{N}$ & $\mathrm{N}$ & $Y$ \\
\hline Bodi ${ }^{17}$ & 2009 & $\mathrm{RL}$ & C & B & c & A & $Y$ & $\mathrm{~N}$ & $Y$ & $Y$ & $Y$ & $Y$ \\
\hline Buelow $^{18}$ & 2014 & $\mathrm{RL}$ & A & A & A & A & $Y$ & $Y$ & $Y$ & $\mathrm{~N}$ & $Y$ & $Y$ \\
\hline Castrioto $^{19}$ & 2015 & $\mathrm{RL}$ & $\mathrm{C}$ & $B$ & $\mathrm{C}$ & B & $Y$ & $Y$ & $\mathrm{~N}$ & $\mathrm{~N}$ & $\mathrm{Y}$ & $\mathrm{Y}$ \\
\hline Cavanagh $^{20}$ & 2017 & $\mathrm{RL}$ & C & A & c & B & $Y$ & $Y$ & $Y$ & $\mathrm{~N}$ & $Y$ & $Y$ \\
\hline Czernecki $^{21}$ & 2002 & $\mathrm{RL}$ & A & B & B & A & $Y$ & $Y$ & $\mathrm{~N}$ & $\mathrm{~N}$ & $Y$ & $Y$ \\
\hline Delazer $^{22}$ & 2009 & $\mathrm{RL}$ & A & A & $\mathrm{C}$ & $A$ & $Y$ & $Y$ & $\mathrm{~N}$ & $\mathrm{~N}$ & $Y$ & $\mathrm{Y}$ \\
\hline Euteneuer $^{23}$ & 2009 & $\mathrm{RL}$ & $\mathrm{C}$ & A & C & $B$ & $\mathrm{Y}$ & $\mathrm{Y}$ & $\mathrm{N}$ & $\mathrm{N}$ & $\mathrm{Y}$ & $\mathrm{Y}$ \\
\hline Evens $^{24}$ & 2015 & $\mathrm{RL}$ & A & A & A & A & $Y$ & $Y$ & $\mathrm{~N}$ & $\mathrm{~N}$ & $Y$ & $Y$ \\
\hline Garofalo $^{25}$ & 2017 & $\mathrm{RL}$ & A & A & C & B & $Y$ & $Y$ & $Y$ & $\mathrm{~N}$ & $Y$ & $Y$ \\
\hline Gescheidt ${ }^{26}$ & 2012 & $\mathrm{RL}$ & A & A & $\mathrm{c}$ & $A$ & $Y$ & $Y$ & $\mathrm{~N}$ & $\mathrm{~N}$ & $Y$ & $Y$ \\
\hline Graef $^{27}$ & 2010 & $\mathrm{RL}$ & A & B & B & A & $Y$ & $Y$ & $\mathrm{~N}$ & $\mathrm{~N}$ & $Y$ & $Y$ \\
\hline Herzallah ${ }^{28}$ & 2017 & RL \& RV & B & A & A & A & $Y$ & $\mathrm{~N}$ & $\mathrm{~N}$ & $\mathrm{~N}$ & $Y$ & $\mathrm{~N}$ \\
\hline Housden $^{29}$ & 2010 & $\mathrm{RL}$ & $\mathrm{C}$ & A & A & $A$ & $Y$ & $Y$ & $\mathrm{Y}$ & $\mathrm{N}$ & $Y$ & $\mathrm{Y}$ \\
\hline Ibarretxe-Bilbao ${ }^{30}$ & 2009 & $\mathrm{RL}$ & A & A & A & B & $Y$ & $Y$ & $\mathrm{~N}$ & $\mathrm{~N}$ & $Y$ & $\mathrm{Y}$ \\
\hline Kobayakawa ${ }^{31}$ & 2008 & RL & $\mathrm{C}$ & A & A & A & $\mathrm{Y}$ & $\mathrm{Y}$ & $\mathrm{N}$ & $\mathrm{N}$ & $\mathrm{Y}$ & $\mathrm{Y}$ \\
\hline Kobayakawa ${ }^{32}$ & 2010 & $\mathrm{RL}$ & $\mathrm{C}$ & A & A & $A$ & $Y$ & $Y$ & $\mathrm{~N}$ & $\mathrm{~N}$ & $Y$ & $\mathrm{Y}$ \\
\hline Mapelli33 & 2014 & $\mathrm{RL}$ & A & A & A & A & $Y$ & $\mathrm{Y}$ & $\mathrm{N}$ & $\mathrm{N}$ & $Y$ & $\mathrm{Y}$ \\
\hline Martinez-Horta ${ }^{34}$ & 2013 & $\mathrm{RL}$ & A & A & $n / a$ & $n / a$ & $Y$ & $\mathrm{~N}$ & $\mathrm{~N}$ & $\mathrm{~N}$ & $Y$ & $Y$ \\
\hline Mimura 35 & 2006 & $\mathrm{RL}$ & $\mathrm{C}$ & A & $B$ & A & $\mathrm{Y}$ & $\mathrm{Y}$ & $\mathrm{N}$ & $\mathrm{N}$ & $\mathrm{Y}$ & $\mathrm{Y}$ \\
\hline Pagonabarraga ${ }^{36}$ & 2007 & $\mathrm{RL}$ & A & A & A & A & $\mathrm{Y}$ & $Y$ & $\mathrm{~N}$ & $\mathrm{~N}$ & $\mathrm{Y}$ & $\mathrm{Y}$ \\
\hline Paz-Alonso ${ }^{37}$ & 2019 & $\mathrm{RL}$ & A & A & A & B & $\mathrm{Y}$ & $Y$ & $\mathrm{Y}$ & $\mathrm{N}$ & $\mathrm{Y}$ & $\mathrm{Y}$ \\
\hline Perretta $^{38}$ & 2005 & $\mathrm{RL}$ & A & A & A & $A$ & $Y$ & $Y$ & $\mathrm{~N}$ & $\mathrm{~N}$ & $Y$ & $\mathrm{Y}$ \\
\hline Pignatt $\mathrm{B}^{39}$ & 2012 & $\mathrm{RL}$ & $A$ & $B$ & $\mathrm{C}$ & $B$ & $\mathrm{~N}$ & $\mathrm{~N}$ & $\mathrm{~N}$ & $\mathrm{~N}$ & $\mathrm{~N}$ & $\mathrm{~N}$ \\
\hline Pineau $^{40}$ & 2016 & $\mathrm{RL}$ & A & A & $n / a$ & $n / a$ & $\mathrm{Y}$ & $\mathrm{Y}$ & $\mathrm{N}$ & $\mathrm{N}$ & $Y$ & $\mathrm{Y}$ \\
\hline Piray 41 & 2014 & $\mathrm{RL}$ & $\mathrm{C}$ & $B$ & C & A & $Y$ & $\mathrm{~N}$ & $\mathrm{Y}$ & $\mathrm{N}$ & $Y$ & $\mathrm{~N}$ \\
\hline Poletti ${ }^{42}$ & 2010 & $\mathrm{RL}$ & A & A & C & $B$ & $\mathrm{Y}$ & $\mathrm{Y}$ & $\mathrm{N}$ & $\mathrm{N}$ & $Y$ & $Y$ \\
\hline Poletti ${ }^{43}$ & 2011 & $\mathrm{RL}$ & A & $B$ & $n / a$ & $n / a$ & $Y$ & $Y$ & $\mathrm{~N}$ & $\mathrm{~N}$ & $\mathrm{~N}$ & $\mathrm{Y}$ \\
\hline Rossi $^{44}$ & 2010 & $\mathrm{RL}$ & A & A & $n / a$ & $n / a$ & $Y$ & $Y$ & $\mathrm{~N}$ & $\mathrm{~N}$ & $Y$ & $\mathrm{Y}$ \\
\hline Sáez-Francàs ${ }^{45}$ & 2014 & $\mathrm{RL}$ & $\mathrm{C}$ & $A$ & $n / a$ & $n / a$ & $Y$ & $Y$ & $\mathrm{~N}$ & $\mathrm{~N}$ & $\mathrm{Y}$ & $\mathrm{N}$ \\
\hline Thiel $^{46}$ & 2003 & $\mathrm{RL}$ & C & A & C & A & $Y$ & $\mathrm{~N}$ & $\mathrm{~N}$ & $\mathrm{~N}$ & $Y$ & $\mathrm{Y}$ \\
\hline Timmer $^{47}$ & 2017 & $\mathrm{RL}$ & A & A & A & $A$ & $Y$ & $Y$ & $Y$ & $\mathrm{~N}$ & $Y$ & $Y$ \\
\hline $\mathrm{Xi}^{48}$ & 2015 & $\mathrm{RL}$ & A & B & $\mathrm{C}$ & B & $Y$ & $Y$ & $\mathrm{~N}$ & $\mathrm{~N}$ & $Y$ & $Y$ \\
\hline
\end{tabular}




\begin{tabular}{|l|l|l|l|l|l|l|l|l|l|l|l|l|} 
Yildirim $^{49}$ & 2020 & RL & A & A & B & A & Y & Y & N & N & Y \\
\hline Drew $^{50}$ & 2020 & RV & A & B & C & A & Y & Y & N & N & Y \\
\hline Evans $^{51}$ & 2010 & RV & A & A & B & B & Y & Y & N & N & Y & Y \\
\hline Lawrence $^{52}$ & 2011 & RV & A & A & n/a & n/a & Y & N & N & N & Y \\
\hline Muhammed $^{53}$ & 2016 & RV & C & A & B & B & Y & Y & Y & N & Y \\
\hline Renfroe $^{54}$ & 2016 & RV & B & B & A & A & Y & Y & N & N & Y \\
\hline Timmer $^{55}$ & 2018 & RV & A & A & A & B & Y & N & Y & N & N \\
\hline
\end{tabular}

\section{References}

1. Bayard, S. et al. A multidimensional approach to impulsivity in Parkinson's disease: measurement and structural invariance of the UPPS Impulsive Behaviour Scale. Psychol. Med. 46, 2931-2941 (2016).

2. J., B. et al. Betting on DBS: Effects of subthalamic nucleus deep brain stimulation on risk taking and decision making in patients with Parkinson's disease. Neuropsychology 29, 622-631 (2015).

3. Chong, T. T. J. et al. Dopamine enhances willingness to exert effort for reward in Parkinson's disease. Cortex 69, 40-46 (2015).

4. Cools, R., Barker, R. A., Sahakian, B. J. \& Robbins, T. W. L-Dopa medication remediates cognitive inflexibility, but increases impulsivity in patients with Parkinson's disease. Neuropsychologia 41, 1431-1441 (2003).

5. B.N., H. et al. Linking brain activity during sequential gambling to impulse control in Parkinson's disease. NeuroImage. Clin. 27, 102330 (2020).

6. Kobayashi, S. et al. Dopaminergic influences on risk preferences of Parkinson's disease patients. Cogn. Affect. Behav. Neurosci. 19, 88-97 (2019).

7. Le Bouc, R. et al. Computational Dissection of Dopamine Motor and Motivational Functions in Humans. J. Neurosci. 36, 6623-6633 (2016).

8. Le Heron, C. et al. Distinct effects of apathy and dopamine on effort-based decision-making in Parkinson's disease. Brain 141, 1455-1469 (2018).

9. S., M. et al. Dopamine restores cognitive motivation in Parkinson's disease. Brain 142, 719-732 (2019).

10. Torta, D. M. E. et al. On the role of dopamine replacement therapy in decision-making, working memory, and reward in Parkinson's disease: does the therapy-dose matter?. Brain Cogn. 71, 84-91 (2009).

11. Sharp, M. E. et al. Decisions under risk in Parkinson's disease: preserved evaluation of probability and magnitude. Neuropsychologia 51, 2679-2689 (2013).

12. Voon, V. et al. Dopamine agonists and risk: Impulse control disorders in Parkinson's; Disease. Brain (2011). doi:10.1093/brain/awr080

13. M., B. et al. Gambling behavior in Parkinson's Disease: Impulsivity, reward mechanism and cortical brain oscillations. Psychiatry Res. 270, 974-980 (2018).

14. Balasubramani, P. P. et al. Identifying the basal ganglia network model markers for medication-induced impulsivity in Parkinson's disease patients. PLoS One 10, e0127542 (2015).

15. Bentivoglio, A. R., Baldonero, E., Ricciardi, L., De Nigris, F. \& Daniele, A. Neuropsychological features of patients with Parkinson's disease and impulse control disorders. Neurol. Sci. (2013). doi:10.1007/s10072-012-1224-5

16. J.W., B. et al. Iowa Gambling Task Performance in Parkinson Disease Patients with Impulse Control Disorders. Arch. Clin. Neuropsychol. 34, 310-318 (2019). 
17. Bodi, N. et al. Reward-learning and the novelty-seeking personality: a between- and within-subjects study of the effects of dopamine agonists on young Parkinson's patients. Brain 132, 2385-2395 (2009).

18. Buelow, M. T. et al. The contribution of apathy and increased learning trials to risky decision-making in Parkinson's disease. Arch. Clin. Neuropsychol. 29, 100-109 (2014).

19. A., C. et al. Iowa gambling task impairment in Parkinson's disease can be normalised by reduction of dopaminergic medication after subthalamic stimulation. J. Neurol. Neurosurg. Psychiatry 86, 186-190 (2015).

20. Cavanagh, J. F. et al. Cognitive states influence dopamine-driven aberrant learning in Parkinson's disease. Cortex (2017). doi:10.1016/j.cortex.2017.02.021

21. V., C. et al. Motivation, reward, and Parkinson's disease: Influence of dopatherapy. Neuropsychologia 40, $2257-2267$ (2002).

22. Delazer, M. et al. Decision making under risk and under ambiguity in Parkinson's disease. Neuropsychologia (2009). doi:10.1016/j.neuropsychologia.2009.02.034

23. Euteneuer, F. et al. Dissociation of decision-making under ambiguity and decision-making under risk in patients with Parkinson's disease: A neuropsychological and psychophysiological study. Neuropsychologia (2009). doi:10.1016/j.neuropsychologia.2009.06.014

24. Evens, R. et al. The impact of Parkinson's disease and subthalamic deep brain stimulation on reward processing. Neuropsychologia (2015). doi:10.1016/j.neuropsychologia.2015.05.005

25. S., G. et al. Cortical and striatal reward processing in Parkinson's disease psychosis. Front. Neurol. 8, 156 (2017).

26. Gescheidt, T. et al. Iowa Gambling Task in patients with early-onset Parkinson's disease: strategy analysis. Neurol. Sci. 33, 1329-1335 (2012).

27. Graef, S. et al. Differential influence of levodopa on reward-based learning in Parkinson's disease. Front. Hum. Neurosci. 4, 169 (2010).

28. Herzallah, M. M. et al. Depression Reduces Accuracy While Parkinsonism Slows Response Time for Processing Positive Feedback in Patients with Parkinson's Disease with Comorbid Major Depressive Disorder Tested on a Probabilistic Category-Learning Task. Front. psychiatry 8, 84 (2017).

29. Housden, C. R. et al. Intact reward learning but elevated delay discounting in Parkinson's disease patients with impulsive-compulsive spectrum behaviors. Neuropsychopharmacology 35, 2155-2164 (2010).

30. Ibarretxe-Bilbao, N. et al. Neuroanatomical correlates of impaired decision-making and facial emotion recognition in early Parkinson's disease. Eur. J. Neurosci. (2009). doi:10.1111/j.1460-9568.2009.06892.x

31. Kobayakawa, M., Koyama, S., Mimura, M. \& Kawamura, M. Decision making in Parkinson's disease: Analysis of behavioral and physiological patterns in the Iowa Gambling task. Mov. Disord. (2008). doi:10.1002/mds.21865

32. Kobayakawa, M., Tsuruya, N. \& Kawamura, M. Sensitivity to reward and punishment in Parkinson's disease: An analysis of behavioral patterns using a modified version of the Iowa gambling task. Park. Relat. Disord. (2010). doi:10.1016/j.parkreldis.2010.04.011

33. Mapelli, D., Di Rosa, E., Cavalletti, M., Schiff, S. \& Tamburin, S. Decision and dopaminergic system: an ERPs study of Iowa gambling task in Parkinson's disease. Front. Psychol. 5, 684 (2014).

34. Martínez-Horta, S., Pagonabarraga, J., Fernández De Bobadilla, R., García-Sanchez, C. \& Kulisevsky, J. Apathy in Parkinson's disease: More than just executive dysfunction. J. Int. Neuropsychol. Soc. (2013). doi:10.1017/S1355617713000131

35. Mimura, M. et al. Impaired decision-making in Parkinson's disease. Parkinsonism Relat. Disord. 12, 169-175 (2006).

36. Pagonabarraga, J. et al. Controlled study of decision-making and cognitive impairment in Parkinson's disease. Mov. Disord. 22, 1430-1435 (2007).

37. Paz-Alonso, P. M. et al. Functional inhibitory control dynamics in impulse control disorders in Parkinson's disease. Mov. Disord. 35, 316-325 (2020). 
38. Perretta, J. G., Pari, G. \& Beninger, R. J. Effects of Parkinson Disease on Two Putative Nondeclarative Learning Tasks. Cogn. Behav. Neurol. (2005). doi:10.1097/01.wnn.0000187939.81541.1d

39. Pignatti, R. et al. Selective IGT decision-making impairment in a patient with juvenile Parkinson's disease and pathological gambling: A role for dopaminergic therapy? Neurocase (2012). doi:10.1080/13554794.2011.633529

40. Pineau, F. et al. Executive functioning and risk-taking behavior in Parkinson's disease patients with impulse control disorders. J. Neural Transm. 123, 573-581 (2016).

41. D., H. et al. Impulse control disorders in Parkinson's disease are associated with dysfunction in stimulus valuation but not action valuation. $J$. Neurosci. 34, 7814-7824 (2014).

42. Poletti, M. et al. Decision making in de novo Parkinson's disease. Mov. Disord. (2010). doi:10.1002/mds.23098

43. Poletti, M. et al. Alexithymia may modulate decision making in patients with de novo Parkinson's disease. Funct. Neurol. 26, 127-131 (2011).

44. Rossi, M. et al. Decision-making in Parkinson's disease patients with and without pathological gambling. Eur. J. Neurol. 17, 97-102 (2010).

45. Saez-Francas, N. et al. Relationship between poor decision-making process and fatigue perception in Parkinson's disease patients. J. Neurol. Sci. 337, 167-172 (2014).

46. Thiel, A. et al. Activation of basal ganglia loops in idiopathic Parkinson's disease: A PET study. J. Neural Transm. (2003). doi:10.1007/s00702-003-0041-7

47. Timmer, M. H. M., Sescousse, G., Van Der Schaaf, M. E., Esselink, R. A. J. \& Cools, R. Reward learning deficits in Parkinson's disease depend on depression. Psychol. Med. (2017). doi:10.1017/S0033291717000769

48. Xi, C. et al. Theory of mind and decision-making processes are impaired in Parkinson's disease. Behav. Brain Res. (2015). doi:10.1016/j.bbr.2014.11.035

49. E., Y. \& S., A. Decision-making and impulse-control disorders in parkinson's disease: Influence of dopaminergic treatment. Neurol. Sci. Neurophysiol. 37, 11-17 (2020).

50. Drew, D. S. et al. Dopamine and reward hypersensitivity in Parkinson's disease with impulse control disorder. Brain 143, $2502-2518$ (2020).

51. A.H., E. et al. Compulsive use of dopaminergic drug therapy in Parkinson's disease: reward and anti-reward. Mov. Disord. 25, 867-876 (2010).

52. Lawrence, A. D., Goerendt, I. K., Brooks, D. J., A.D., L. \& I.K., G. Apathy blunts neural response to money in Parkinson's disease. Soc. Neurosci. 6, 653-662 (2011).

53. Muhammed, K. et al. Reward sensitivity deficits modulated by dopamine are associated with apathy in Parkinson's disease. Brain 139, 27062721 (2016).

54. J.B., R. et al. Motivational engagement in Parkinson's disease: Preparation for motivated action. Int. J. Psychophysiol. 99, 24-32 (2016).

55. M.H.M., T. et al. Enhanced motivation of cognitive control in Parkinson's disease. Eur. J. Neurosci. 48, $2374-2384$ (2018). 